\title{
Wave Number-Frequency Domain Properties of Coastal Sea Level Response to Alongshore Wind Stress Along the West Coast of North America 1980-1984
}

\author{
George R. Halliwell, JR. ${ }^{1}$ AND J. S. Allen \\ College of Oceanography, Oregon State University, Corvalls
}

\begin{abstract}
Fluctuations in alongshore wind stress $\tau$ calculated from Fleet Numerical Oceanography Center geostrophic winds along the west coast of North America are relatively energetic at alongshore wave numbers $|l| \leq 11 \times 10^{-3} \mathrm{cpkm}$ and frequencies $0.025<\omega<0.5 \mathrm{cpd}$, and they effectively drive a coastal sea level $(\zeta)$ response within this $(l, \omega)$ band. Equatorward propagation $(l<0)$ dominates the winter $\tau$ fluctuations for $\omega<0.35 \mathrm{cpd}$, and poleward propagation $(l>0)$ dominates the summer $\tau$ fluctuations for $\omega<0.1 \mathrm{cpd}$. In contrast, poleward propagation strongly dominates the $\zeta$ fluctuations in both seasons, but more so in summer. The largest coherence between $\zeta$ and $\tau$ is observed in that part of $(l, \omega)$ space where $\tau$ is most energetic: i.e., for $0 \leq l \leq 1.1 \times 10^{-3} \mathrm{cpkm}$ in summer and $-1.1 \times 10^{-3} \leq l \leq 0 \mathrm{cpkm}$ in winter. Results are compared with predictions from theoretical models for wind-driven coastally trapped waves. The observed transfer functions show evidence for near-resonant $\zeta$ response in both seasons, with the largest gain found along a single ridge of approximately constant poleward phase speed, along which the response is approximately in phase with $\tau$. This behavior is consistent with a response dominated by one coastally trapped wave mode governed by a forced, first-order wave equation with a linear friction term. The maximum gain tends to decrease along this ridge with increasing $l$ and $\omega$ in summer, and at least with increasing $\omega$ in winter, during which we could not resolve the $l$ dependence. This observed decrease in maximum gain is not predicted by a single wave equation. The contribution of higher wave modes to the total $\zeta$ response may explain a part of this observed decrease.
\end{abstract}

\section{INTRODUCTION}

Considerable observational and theoretical evidence has accumulated indicating that free perturbations in current and pressure over a continental shelf propagate alongshore with the coast to the right (left) in the northern (southern) hemisphere [e.g., Allen, 1980]. Based on scaling arguments in the equations of motion, the alongshore component of wind stress at the coast $(\tau)$ is the most important atmospheric forcing variable for perturbations with large alongshore scales ( $\gg$ shelf-slope width). Halliwell and Allen [1984] showed that the coastal ocean response tends to be larger for $\tau$ disturbances that propagate alongshore in the same direction as free coastal sea level $(\zeta)$ perturbations as opposed to $\tau$ disturbances that occur at a fixed location along the coast or propagate alongshore in the opposite direction. The coastal ocean response therefore depends in part on the alongshore wave number and frequency domain properties of the $\tau$ field.

Since the observed properties of this response have never been studied in wave number-frequency space, we sought, as part of the large-scale component of the Coastal Ocean Dynamics Experiment (CODE), to characterize wave numberfrequency domain properties of the $\tau$ and $\zeta$ fields, and of the $\zeta$ response to $\tau$. We focus primarily on the large-scale (smallwave number) properties during four spring-summer upwelling seasons and four winter seasons between May 1980 and March 1984, primarily using wave number-frequency autospectrum and cross-spectrum analysis. We describe the ob-

\footnotetext{
${ }^{1}$ Now at Graduate School of Oceanography, University of Rhode Island, Narragansett.
}

Copyright 1987 by the American Geophysical Union.

Paper number 7C0509.

0148-0227/87/007C-0509\$05.00 served properties and relate them to properties predicted from simple coastal circulation models.

The simplest linear models predict that the coastal circulation response to variable alongshore wind stress with large alongshore scales consists of an infinite number of coastally trapped wave modes, with the alongshore-time variability of each mode governed by a forced, first-order wave equation. Given an alongshore-time $(y, t)$ coordinate system, where $y$ is positive poleward along the west coast of North America, the wave equation for mode $n$ of a given coastal circulation response variable $Y_{n}(y, t)$ is

$$
c_{n}^{-1} Y_{n t}+Y_{n y}+\left(c_{n} T_{f n}\right)^{-1} Y_{n}=b_{n} \tau
$$

where $c_{n}$ are the free-wave phase speeds, $T_{f n}$ are the friction time scales, and $b_{n}$ are the coupling coefficients. These modal equations (without the friction term) were first derived by Gill and Schumann [1974] tor a barotropic fluid over a continental shelf and by Gill and Clarke [1974] for coastal Kelvin waves in a stratified fluid. Clarke [1977] derived similar frictionless equations for a continental shell with horizontally uniform basic stratification. Major simplifying assumptions, such as neglecting the alongshore dependence of bottom topography, must be made in these derivations. The linear friction term in (1) was added in an ad hoc manner in these early studies. Brink [1982] showed that if friction is sufficiently strong, each mode becomes frictionally coupled to all other modes.

In previous studies where one mode was assumed to dominate the response, (1) was found to predict the $\zeta$ response at a given location along the coast with reasonably good accuracy [Hamon, 1976; Clarke, 1977; Halliwell and Allen, 1984]. It was also found to predict the nature of certain observed statistical relationships between the response and $\tau$ [Allen and Denbo, 1984]. In the present study, we determine that a single wave equation (1) qualitatively predicts many, but not all, of the 


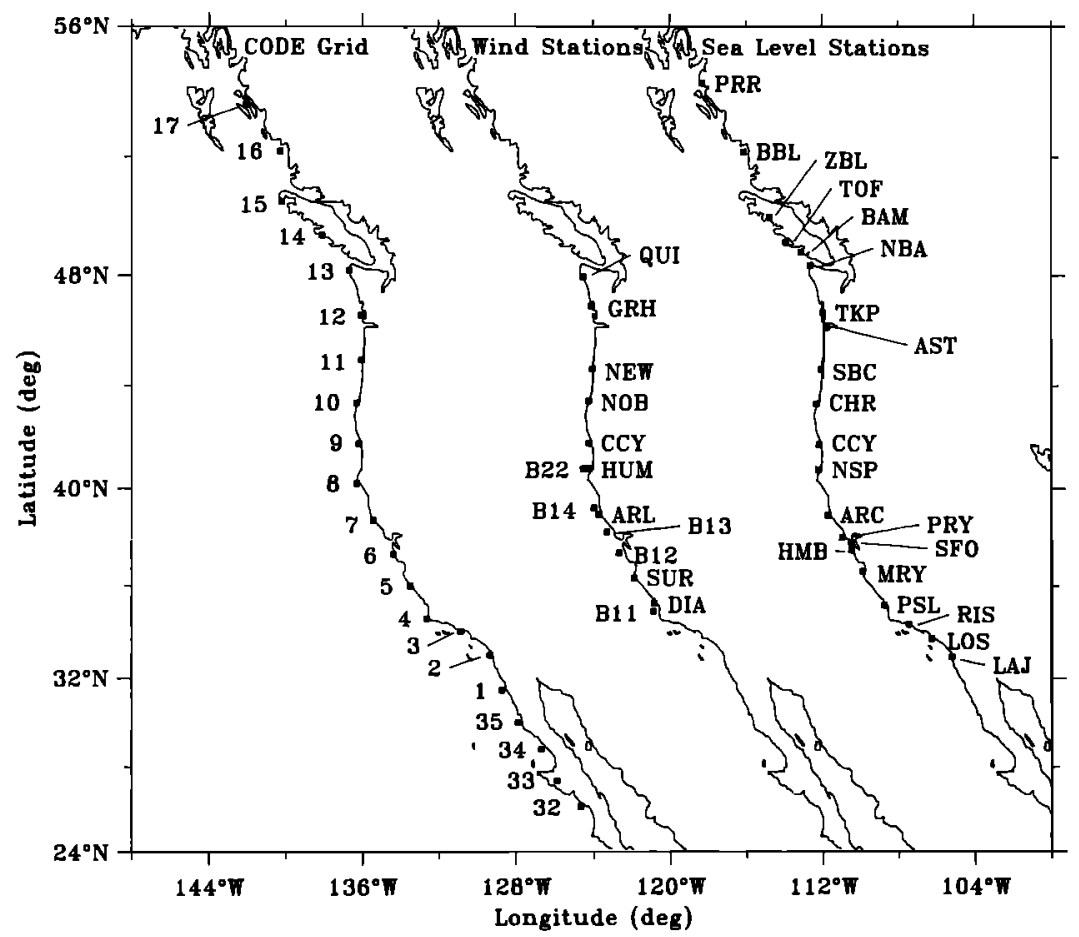

Fig. 1. The coastal points of the CODE analysis grid (left coastline), the wind measurement stations (middle coastline), and the coastal sea level stations (right coastline) used in this study. The longitude axis is correct for the middle coastline. Information on the stations and grid points is summarized in Tables 1 through 3.

observed properties of the $\zeta$ response to $\tau$ in wave numberfrequency space. We show that the properties not predicted by a single wave equation can at least in part be explained by the contribution of higher modes to the total response.

We first describe the $\tau$ and $\zeta$ data sets and discuss certain properties of these sets that may affect our analyses (section 2). We then discuss the basic statistical properties of the $\tau$ and $\zeta$ fields in summer and winter, comparing them to observations reported by Halliwell and Allen [1984] for summer 1973 and to properties predicted from (1) by Allen and Denbo [1984] (section 3). We then present the $\zeta$ and $\tau$ wave numberfrequency autospectra (section 4) and discuss the observed properties of the $\zeta$ response to $\tau$ in wave number-frequency space (section 5), emphasizing the similarities and differences in the observed properties of the forcing and response between summer and winter, plus the interannual variability in these properties for each season. Finally, we compare the observed response properties in wave number-frequency space to those predicted by (1) (section 6). In all cases, when we refer to responses predicted from (1), we mean from a single wave equation unless we explicitly state otherwise.

\section{THE DATA}

We analyze the response of $\zeta$ to $\tau$ within a $2700 \mathrm{~km}$ domain along the west coast of North America, stretching approximately from San Diego $\left(32.7^{\circ} \mathrm{N}\right)$ to near the Alaska-British Columbia border $\left(54.1^{\circ} \mathrm{N}\right)$. We use an alongshore-time $(y, t)$ coordinate system, with the $y=0 \mathrm{~km}$ coordinate located at the central line of the intensive CODE experiments $\left(38.7^{\circ} \mathrm{N}\right.$, hereinafter referred to as the CODE site) [Winant et al., 1987], and analyze data interpolated to points of the CODE analysis grid (Figure 1, Table 1). Grid point 7 is located at $y=0 \mathrm{~km}$, and the alongshore spacing of the points is $\Delta y=180 \mathrm{~km}$. Our common analysis domain for both $\zeta$ and $\tau$ includes grid points 2 through $17(-900 \leq y \leq 1800 \mathrm{~km})$ since no coastal sea level data were available from point 1 south. However, $\tau$ is also available at grid points south to central Baja California (grid point $32 ; y=-1800 \mathrm{~km}$ ). We study the following four spring-

TABLE 1. Coastal Points of the CODE Large-Scale Analysis Grid

\begin{tabular}{ccccc}
\hline $\begin{array}{c}\text { Grid } \\
\text { Point }\end{array}$ & $\begin{array}{c}\text { Latitude, } \\
{ }^{\circ} \mathbf{N}\end{array}$ & $\begin{array}{c}\text { Longitude, } \\
{ }^{\circ} \mathbf{W}\end{array}$ & $\begin{array}{c}y, \\
\mathbf{k m}\end{array}$ & $\begin{array}{c}\text { Coast } \\
\text { Orientation, } \\
\text { deg }\end{array}$ \\
\hline 17 & $54^{\circ} 09^{\prime}$ & $130^{\circ} 20^{\prime}$ & 1800 & 120 \\
16 & $52^{\circ} 10^{\prime}$ & $128^{\circ} 19^{\prime}$ & 1620 & 115 \\
15 & $50^{\circ} 32^{\prime}$ & $127^{\prime} 13^{\prime}$ & 1440 & 100 \\
14 & $49^{\circ} 23^{\prime}$ & $126^{\circ} 06^{\prime}$ & 1260 & 128 \\
13 & $4^{\circ} 11^{\prime}$ & $124^{\circ} 42^{\prime}$ & 1080 & 120 \\
12 & $4^{\circ} 36^{\prime}$ & $124^{\circ} 05^{\prime}$ & 900 & 95 \\
11 & $4^{\circ} 58^{\prime}$ & $124^{\circ} 03^{\prime}$ & 720 & 85 \\
10 & $4^{\circ} 21^{\prime}$ & $124^{\circ} 20^{\prime}$ & 540 & 73 \\
09 & $41^{\circ} 46^{\prime}$ & $124^{\circ} 12^{\prime}$ & 360 & 103 \\
08 & $40^{\circ} 12^{\prime}$ & $124^{\circ} 18^{\prime}$ & 180 & 130 \\
07 & $38^{\circ} 42^{\prime}$ & $123^{\prime \prime} 27^{\prime}$ & 0 & 133 \\
06 & $37^{\circ} 18^{\prime}$ & $122^{\circ} 24^{\prime}$ & -180 & 102 \\
05 & $35^{\circ} 59^{\prime}$ & $121^{\circ} 31^{\prime}$ & -360 & 128 \\
04 & $34^{\circ} 35^{\prime}$ & $120^{\circ} 39^{\prime}$ & -540 & 90 \\
03 & $34^{\circ} 01^{\prime}$ & $118^{\circ} 53^{\prime}$ & -720 & 150 \\
02 & $33^{\circ} 00^{\prime}$ & $117^{\circ} 21^{\prime}$ & -900 & 110 \\
01 & $31^{\circ} 27^{\prime}$ & $116^{\circ} 44^{\prime}$ & -1080 & 110 \\
35 & $30^{\circ} 00^{\prime}$ & $115^{\circ} 54^{\prime}$ & -1260 & 115 \\
34 & $28^{\circ} 47^{\prime}$ & $114^{\circ} 41^{\prime}$ & -1440 & 130 \\
33 & $27^{\circ} 20^{\prime}$ & $113^{\circ} 52^{\prime}$ & -1620 & 130 \\
32 & $26^{\circ} 07^{\prime}$ & $112^{\circ} 40^{\prime}$ & -1800 & 130 \\
\hline
\end{tabular}

Coast orientation is measured in degrees counterclockwise from due east. 
TABLE 2. Coastal Sea Level Stations Used in This Analysis

\begin{tabular}{llllr}
\hline \multicolumn{1}{c}{ Station } & $\begin{array}{c}\text { Abbrevi- } \\
\text { ation }\end{array}$ & $\begin{array}{c}\text { Latitude, } \\
{ }^{\circ} \mathbf{N}\end{array}$ & $\begin{array}{c}\text { Longitude, } \\
{ }^{\circ} \mathbf{W}\end{array}$ & $\begin{array}{r}y, \\
\mathbf{k m}\end{array}$ \\
\hline Prince Rupert, B. C. & PRR & $54^{\circ} 19^{\prime}$ & $130^{\circ} 20^{\prime}$ & 1883 \\
Bella Bella, B. C. & BBL & $52^{\circ} 10^{\prime}$ & $128^{\circ} 08^{\prime}$ & 1608 \\
Zeballos, B. C. & ZBL & $50^{\circ} 01^{\prime}$ & $126^{\circ} 47^{\prime}$ & 1349 \\
Tofino, B. C. & TOF & $49^{\circ} 09^{\prime}$ & $125^{\circ} 55^{\prime}$ & 1232 \\
Neah Bay, Wash. & NBA & $48^{\circ} 22^{\prime}$ & $124^{\circ} 37^{\prime}$ & 1100 \\
Toke Point, Wash. & TKP & $46^{\circ} 42^{\prime}$ & $123^{\circ} 58^{\prime}$ & 911 \\
Astoria Oreg. & AST & $46^{\circ} 10^{\prime}$ & $123^{\circ} 46^{\prime}$ & 852 \\
South Beach, Oreg. & SBC & $44^{\circ} 38^{\prime}$ & $124^{\circ} 03^{\prime}$ & 683 \\
Charleston, Oreg. & CHR & $43^{\circ} 20^{\prime}$ & $124^{\circ} 19^{\prime}$ & 538 \\
Crescent City, Calif. & CCY & $41^{\circ} 45^{\prime}$ & $124^{\circ} 11^{\prime}$ & 359 \\
Trinidad Head, Calif. & TRH & $41^{\circ} 03^{\prime}$ & $124^{\circ} 09^{\prime}$ & 277 \\
North Spit, Calif. & NSP & $40^{\circ} 45^{\prime}$ & $124^{\circ} 14^{\prime}$ & 244 \\
Arena Cove, Calif. & ARC & $38^{\circ} 55^{\prime}$ & $123^{\circ} 43^{\prime}$ & 37 \\
Point Reyes, Calif. & PRY & $38^{\circ} 00^{\prime}$ & $122^{\circ} 58^{\prime}$ & -76 \\
San Francisco, Calif. & SFO & $37^{\circ} 48^{\prime}$ & $122^{\circ} 28^{\prime}$ & -126 \\
Monterey, Calif. & MRY & $36^{\circ} 36^{\prime}$ & $121^{\circ} 53^{\prime}$ & -276 \\
Port San Luis, Calif. & PSL & $35^{\circ} 10^{\prime}$ & $120^{\circ} 45^{\prime}$ & -473 \\
Santa Barbara, Calif. & SBA & $34^{\circ} 25^{\prime}$ & $119^{\circ} 41^{\prime}$ & -634 \\
Rincon Island, Calif. & RIS & $34^{\circ} 21^{\prime}$ & $119^{\circ} 27^{\prime}$ & -650 \\
Los Angeles, Calif. & LOS & $33^{\circ} 43^{\prime}$ & $118^{\circ} 16^{\prime}$ & -780 \\
San Diego, Calif. & SDO & $32^{\circ} 45^{\prime}$ & $117^{\circ} 10^{\prime}$ & -938 \\
\hline
\end{tabular}

summer (hereinafter referred to as summer) and four winter seasons, all of which are 121 days long: (1) May 1 to August 29, 1980; (2) April 4 to August 2, 1981 (CODE 1 experiment); (3) May 1 to August 29, 1982 (CODE 2 experiment); (4) May 1 to August 29, 1983; (5) November 25, 1980, to March 25, 1981 ; (6) December 1, 1981, to March 31, 1982; (7) December 1, 1982, to March 31,1983; and (8) November 27, 1983, to March 26, 1984. We refer to the individual seasons as summers 1980 through 1983 and winters 1980-1981 through 1983-1984. All summer seasons begin after the spring transition [Strub et al., 1987], which occurs between late March and mid-April. All winter seasons are chosen to terminate before the transition, which explains their staggered times. All summer seasons begin on May 1 except for summer 1981, which is shifted to coincide better with the extensive instrument deployments of the CODE 1 experiment [Winant et al., 1987].
Coastal sea level data are obtained at several stations (Figure 1 and Table 2) for all eight seasons. Sea level time series are checked for bad values and datum shifts [Pittock et al., 1982]. Details of the remaining editorial procedure are given by Halliwell et al. [1986]. To create $\zeta$ time series, sea level heights are adjusted to represent coastal subsurface pressure by adding Fleet Numerical Oceanography Center (FNOC) surface atmospheric pressure in millibars from the nearest CODE grid point to sea level in centimeters if that point is within $30 \mathrm{~km}$ of the sea level station. II it is farther away, atmospheric pressure linearly interpolated between the two nearest grid points is used. The mean $\zeta$ is removed from all time series for each individual season. We use $\zeta$ as the response variable because it is available over a sufficiently large alongshore domain to resolve some of the large scales present in the coastal ocean response along the west coast of North America.

We compute $\tau$ time series using geostrophic winds obtained from FNOC [Bakun, 1975; Halliwell and Allen, 1987] and interpolated to the CODE analysis grid. Hereinafter, we refer to this field as calculated $\tau$. The coarse spatial resolution of calculated $\tau$ effectively restricts its use to represent fluctuations with wavelengths greater than several hundred kilometers. Calculated $\tau$ is used for the 4-year study interval because the highest-quality CODE large-scale measured wind data set spans the interval 1981-1982 and because calculated winds are readily obtainable over a larger alongshore domain than measured winds. The properties of, and relationships between, the calculated and measured wind fields are discussed by Halliwell and Allen [1987]. To assess the effects of using calculated $\tau$ in the present analysis, we study properties of wave numberfrequency auto-spectra and cross-spectra of calculated $\tau$, measured $\tau$, and $\zeta$ using measured winds from several stations (Figure 1 and Table 3) for summers 1981 and 1982, plus winter 1981-1982 (sections 4.3 and 5.3). Information on coastal stations, details of data processing, and basic visual and statistical summaries of the entire large-scale data set are presented by Halliwell and Allen [1983, 1985] and Halliwell et al. [1986].

Vector wind stress is computed from vector wind $10 \mathrm{~m}$

TABLE 3. Wind Measurement Stations Used in the Intercomparison With Calculated Winds

\begin{tabular}{llccrrr}
\hline \multicolumn{1}{c}{ Station } & $\begin{array}{c}\text { Abbrevi- } \\
\text { ation }\end{array}$ & $\begin{array}{c}\text { Latitude, } \\
{ }^{\circ} \mathrm{N}\end{array}$ & $\begin{array}{c}\text { Longitude, } \\
{ }^{\circ} \mathbf{W}\end{array}$ & $\begin{array}{c}y, \\
\mathrm{~km}\end{array}$ & $\begin{array}{c}\text { Coast } \\
\text { Orientation, } \\
\text { deg }\end{array}$ & $\begin{array}{r}\text { Time } \\
\text { Periods } \\
\text { Used }\end{array}$ \\
\hline Quillayute, Wash. & QUI & $47^{\circ} 57^{\prime}$ & $124^{\circ} 32^{\prime}$ & 1055 & 110 & All \\
Grays Harbor, Wash. & GRH & $4^{\circ} 55^{\prime}$ & $124^{\circ} 06^{\prime}$ & 936 & 95 & All \\
Newport, Oreg. & NEW & $4^{\circ} 38^{\prime}$ & $124^{\circ} 03^{\prime}$ & 683 & 82 & All \\
North Bend, Oreg. & NOB & $4^{\circ} 25^{\prime}$ & $124^{\circ} 15^{\prime}$ & 547 & 73 & All \\
Crescent City, Calif. & CCY & $4^{\circ} 47^{\prime}$ & $124^{\circ} 14^{\prime}$ & 362 & 103 & All \\
Humboldt Bay, Calif. & HUM & $4^{\circ} 46^{\prime}$ & $124^{\circ} 14^{\prime}$ & 246 & 75 & S81 \\
NDBC 46022 & B22 & $4^{\circ} 46^{\prime}$ & $124^{\circ} 31^{\prime}$ & 240 & 75 & W81-2, S82 \\
NDBC 46014 & B14 & $39^{\circ} 13^{\prime}$ & $123^{\circ} 58^{\prime}$ & 71 & 100 & S81, S82 \\
Point Arena Light, Calif. & ARL & $38^{\circ} 57^{\prime}$ & $123^{\circ} 44^{\prime}$ & 41 & 110 & W81-2 \\
NDBC 46013 & B13 & $38^{\circ} 14^{\prime}$ & $123^{\circ} 18^{\prime}$ & -61 & 133 & All \\
NDBC 46012 & B12 & $37^{\circ} 22^{\prime}$ & $122^{\circ} 39^{\prime}$ & -171 & 105 & All \\
Point Sur, Calif. & SUR & $36^{\circ} 18^{\prime}$ & $121^{\circ} 53^{\prime}$ & -301 & 115 & All \\
Diablo Canyon, Calif. & DIA & $35^{\circ} 14^{\prime}$ & $120^{\circ} 50^{\prime}$ & -464 & 120 & W81-2 \\
NDBC 46011 & B11 & $34^{\circ} 53^{\prime}$ & $120^{\circ} 52^{\prime}$ & -506 & 90 & S81, S82 \\
Point Mugu, Calif. & MUG & $34^{\circ} 07^{\prime}$ & $119^{\circ} 07^{\prime}$ & -678 & 155 & All \\
San Diego, Calif. & SDO & $32^{\circ} 44^{\prime}$ & $117^{\circ} 10^{\prime}$ & -936 & 105 & All \\
\hline
\end{tabular}

Coast orientation is in degrees counterclockwise from due east. NDBC stands for National Data Buoy Center. 
above the surface $\left(V_{10}\right)$ in units of meters per second, using the bulk aerodynamic formula with the drag coefficient determined from the regression formula of Large and Pond [1981]:

$$
\tau=\rho_{a} C_{d}\left|\mathbf{V}_{10}\right| \mathbf{V}_{10}
$$

with

$$
\begin{array}{ll}
10^{3} C_{d}=1.2 & \left|\mathbf{V}_{10}\right| \leq 11 \\
10^{3} C_{d}=0.49+0.065\left|\mathbf{V}_{10}\right| & \left|\mathbf{V}_{10}\right|>11
\end{array}
$$

where $\rho_{a}$ is the air density and $C_{d}$ is the drag coefficient. Where necessary, measured winds are adjusted to represent 10-m winds assuming neutral stability [Halliwell et al., 1986]. We assume that the calculated winds received from FNOC represent $10-\mathrm{m}$ winds. Before computing the alongshore component of the wind stress, the calculated vector wind stress is rotated as was described by Halliwell and Allen [1987] to improve the boundary layer correction of Bakun [1975]. We use the geographical alongshore component of the measured vector wind stress. All $\tau$ and $\zeta$ time series are low-pass filtered with a half-amplitude period of 40 hours, and the time sampling rate is $\Delta t=0.25$ days.

Wind velocity usually varies in the across-shore direction over the continental shelf, and may also be directly influenced by local topography at the coast. The measured wind set consists of a mixture of coastal land stations and offshore buoys. Because of these factors, the variance of measured and calculated alongshore winds differs substantially at many locations along the coast. Halliwell and Allen [1987] found that it was not possible to adjust measured coastal land winds to represent winds over the outer continental shelf within the CODE large-scale domain in a consistent manner. They also found, however, that properties of wave number-frequency autospectra and cross-spectra of alongshore wind were not sensitive to the distribution of wind variance along the coast. Since $\tau$ is a nonlinear function of $V_{10}$, and since the drag coeflicient is a function of wind speed if it exceeds $11 \mathrm{~m} \mathrm{~s}^{-1}$, any differences in alongshore wind variance along the coast will be amplified in $\tau$. We therefore felt that it was necessary to repeat these tests in this paper for the $\tau$ field. We do this by including analysis results for adjusted measured $\tau$ wherever we compare analysis results for calculated $\tau$ to those of measured $\tau$. To form adjusted measured $\tau$, we simply scale the amplitude of measured $\tau$ so that its variance equals the calculated $\tau$ variance at that alongshore location. (We do not intend to imply by this choice that the calculated $\tau$ field represents the alongshore distribution of $\tau$ variance more accurately than the measured $\tau$ field.) Comparison of results obtained from the different $\tau$ fields allows us to assess the effects of errors in measuring the alongshore distribution of $\tau$ variance.

\section{Basic Statistical Properties of the $\tau$ AND $\zeta$ FIELDS}

\subsection{Means and rms Amplitudes}

In all analyses presented in this paper, summer and winter statistics refer to statistics averaged over the four summer and four winter seasons. We will always use $\tau$ to refer to calculated $\tau$ and will use the phrase "calculated $\tau$ " only when it must be distinguished from measured $\tau$.

Substantial differences in the basic statistics of the $\tau$ and $\zeta$ fields exist between summer and winter. The mean of $\tau$ is

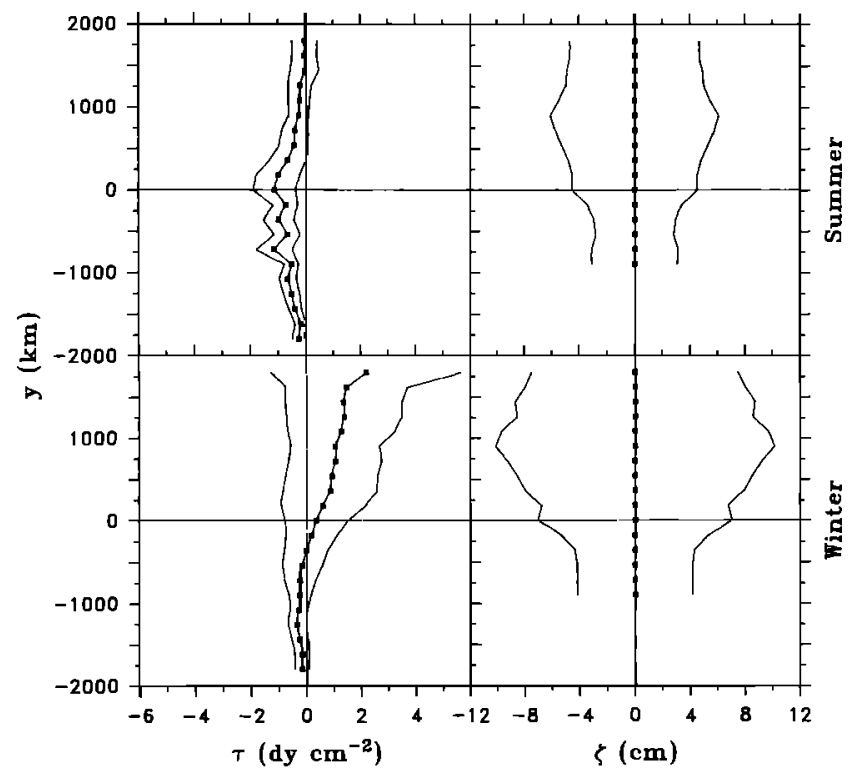

Fig. 2. The mean (solid line with squares) and rms amplitude envelopes of $\tau$ and $\zeta$ for summer and winter. The means and rms amplitudes for both seasons are the averages of the means and rms amplitudes of the individual summer and winter seasons. The $\tau$ statistics south of grid point 2 are also included, and $\zeta$ has been interpolated to the CODE grid points. The means of $\zeta$ have been removed.

negative (equatorward) within the entire CODE large-scale domain in summer but is negative only for $y \leq-360 \mathrm{~km}$ in winter (Figure 2). The magnitude of the summer mean is largest within the alongshore subdomain $-720<y<180 \mathrm{~km}$,

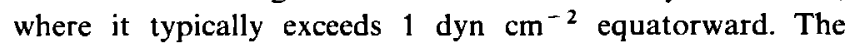
summer mean calculated $\tau$ does not accurately represent the actual mean within the Southern California Bight $(-1000<$ $y<-575 \mathrm{~km}$ ), since calculated alongshore and vector wind means differ substantially from the corresponding measured wind means within the bight in summer [Halliwell and Allen, 1987]. The winter equatorward means for $y<-360 \mathrm{~km}$ are

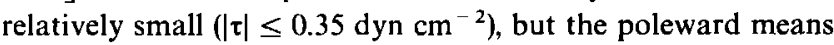
are substantially larger for $y \geq 180 \mathrm{~km}$, exceeding $2 \mathrm{dyn}^{-2}$ at the northern end of the CODE large-scale domain. The observed properties of seasonal mean $\tau$ generally agree with seasonal differences in the properties of the alongshore component of monthly mean wind stress near the coast computed

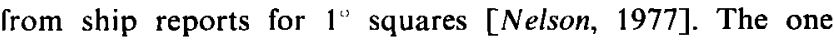
major difference is that mean calculated $\tau$ changes sign in winter near $y=-360 \mathrm{~km}$, while Nelson's data indicate the sign change is near Cape Mendocino $(y \approx 200 \mathrm{~km})$ during winter months.

For summer, the largest rms amplitude of $\tau$ exists in the subdomain $-720<y<180 \mathrm{~km}$, the same subdomain where mean equatorward $\tau$ is largest (Figure 2). The maximum summer rms amplitude is $0.79 \mathrm{dyn}^{-2}$ at $y=180 \mathrm{~km}$ (near Cape Mendocino). The rms amplitude generally increases for increasing $y$ in winter. The winter rms amplitude increases substantially poleward from $y=-900 \mathrm{~km}\left(0.32 \mathrm{dyn}^{-2}\right)$ to a relative maximum at $y=360 \mathrm{~km}\left(1.72 \mathrm{dyn} \mathrm{cm}^{-2}\right)$. It is approximately constant near $1.65 \mathrm{dyn} \mathrm{cm}^{-2}$ in the subdomain $540 \leq y \leq 900 \mathrm{~km}$. It then increases rapidly poleward of $y=900 \mathrm{~km}$ to $3.48 \mathrm{dyn}^{-2}$ at $y=1800 \mathrm{~km}$. The rms amplitude of $\zeta$ is also substantially greater in winter than in summer, but in contrast to $\tau$, similar patterns in the alongshore distributions of $\zeta \mathrm{rms}$ amplitudes are observed in both 


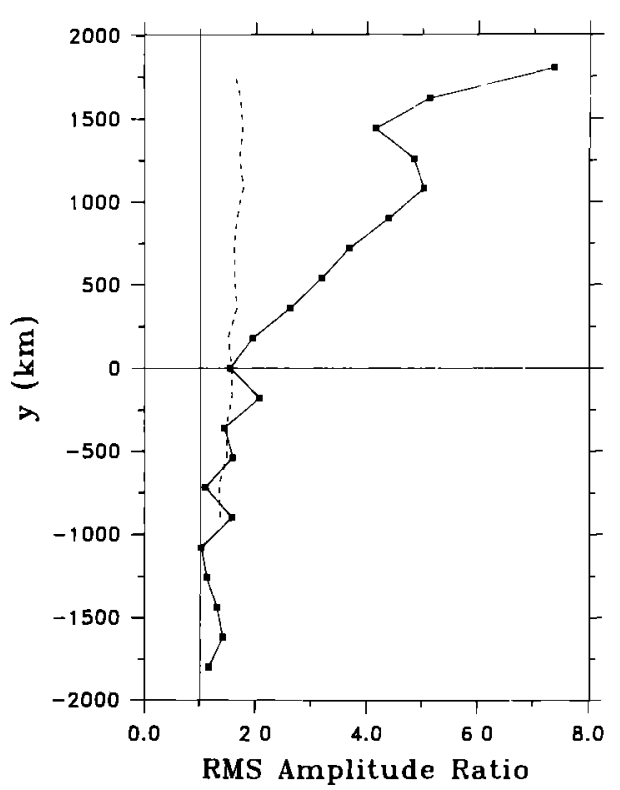

Fig. 3. The ratios of winter rms amplitude to summer rms amplitude for $\tau$ (solid line with squares) and $\zeta$ (dashed line) which has been interpolated to the CODE grid. A ratıo of 1.0 is indicated by the vertical solid line.

seasons (Figure 2). In both summer and winter, the $\zeta$ amplitudes are roughly constant for $y<-360 \mathrm{~km}$, increase with increasing $y$ for $-360 \leq y \leq 900 \mathrm{~km}$, and then decrease with increasing $y$ for $y>900 \mathrm{~km}$. The summer poleward increase is from $2.91 \mathrm{~cm}$ at $y=-360 \mathrm{~km}$ to $6.10 \mathrm{~cm}$ at $y=900 \mathrm{~km}$. The corresponding winter increase is from 4.27 to $10.15 \mathrm{~cm}$.

The strikingly different seasonal changes in the rms amplitudes of $\tau$ and $\zeta$ are evident from the ratios of winter to summer rms amplitude plotted as a function of $y$ (Figure 3).
Equatorward of the CODE site, the amplitude of $\tau$ is between 1 and 1.6 times larger, and the amplitude of $\zeta$ is between 1.35 and 1.5 larger, in winter than in summer. Poleward of the CODE site, the ratio for $\zeta$ increases slightly to values typically between 1.6 and 1.75 , but the ratio for $\tau$ increases very sharply, reaching 5.0 at a relative maximum at $y=1080 \mathrm{~km}$, and then reaching 7.4 at $y=1800 \mathrm{~km}$. Therefore the increases in $\tau$ and $\zeta$ rms amplitudes in winter over summer have similar magnitudes equatorward of the CODE site, but the $\tau$ increase becomes much larger than the $\zeta$ increase poleward of the CODE site. This winter increase depends substantially on the choice of $C_{\mathrm{d}}$. Calculated wind speeds exceeding $11 \mathrm{~m} \mathrm{~s}^{-1}$, above which $C_{d}$ from (3) increases with wind speed, are uncommon in summer but occur more frequently in winter, especially to the north of the CODE site. The ratio plotted in Figure 3 for $\tau$ decreases by about $50 \%$ at grid point 17 $(y=1800 \mathrm{~km})$ if a constant $C_{d}$ is used. This winter increase is statistically significant whether $C_{d}$ is constant or increases with wind speed, but the magnitude of the ratio does depend on $C_{d}$. We address possible causes of this $y$-dependent seasonal change in our discussion of the wave number-frequency spectrum analyses.

\subsection{Correlation/Coherence Scales and Alongshore Propagation}

We estimate the correlation space and times scales, plus the alongshore propagation properties, of the $\tau$ and $\zeta$ fluctuations using the spatially averaged, space-time auto-correlation and cross-correlation functions $\hat{R}_{\pi \tau}\left(\eta_{n}, t_{l}\right), \hat{R}_{\zeta \zeta}\left(\eta_{n}, t_{l}\right)$, and $\hat{R}_{\zeta \tau}\left(\eta_{n}, t_{l}\right)$, calculated for both summer (grid points 4 through 17) and winter (grid points 2 through 17) (Figure 4). We use the circumflex to signify quantities estimated from the data. Grid points 2 and 3 are not included in summer, since calculated winds poorly represent coastal winds in the Southern Califor-

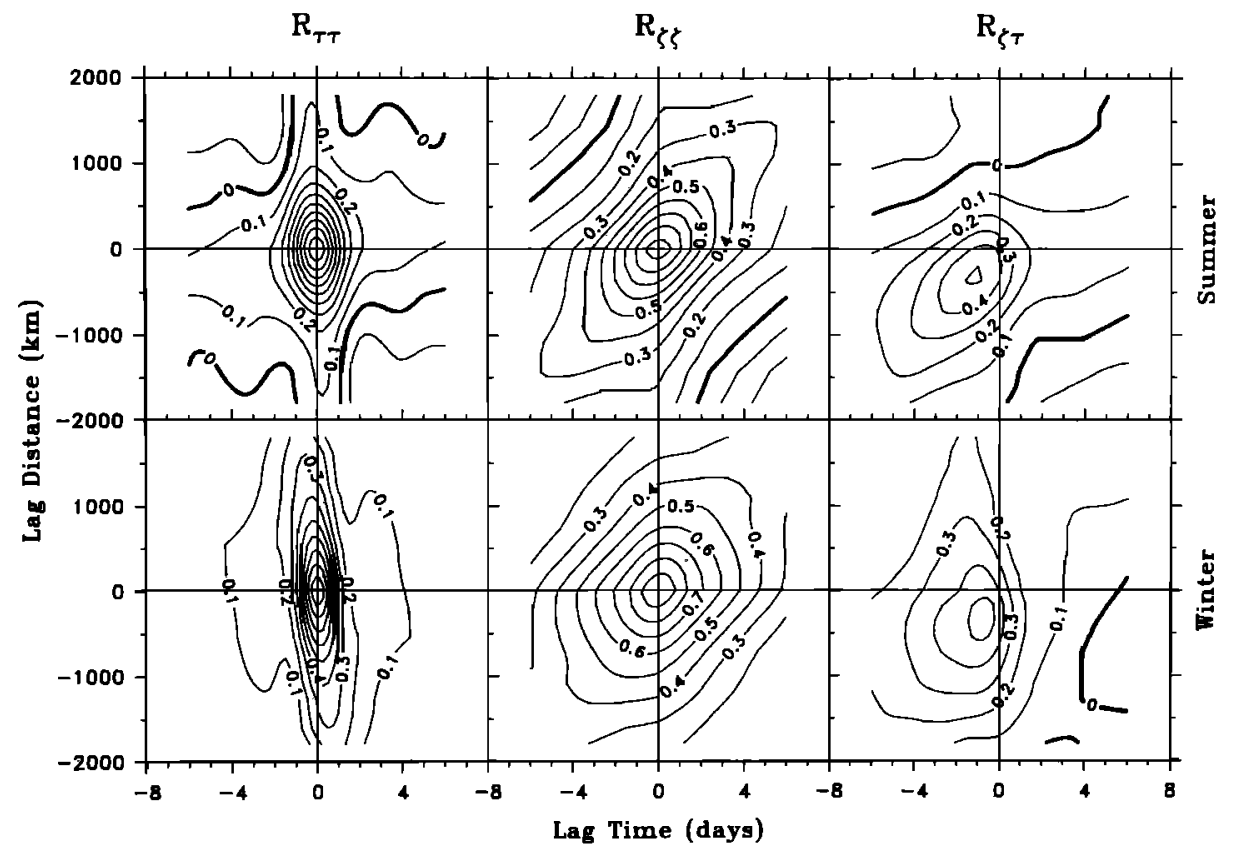

Fig. 4. The space-time correlation functions $\hat{R}_{r \tau}\left(\eta_{n}, \tau_{l}\right), \hat{R}_{t \zeta}\left(\eta_{n}, \tau_{l}\right)$, and $\hat{R}_{\zeta \tau}\left(\eta_{n}, \tau_{l}\right)$, calculated from space-averaged covariances as described in Appendix $A$ and ensemble averaged over the four summers (grid points 4 through 17) and four winters (grid points 2 through 17). 
TABLE 4. Integral Space and Time Correlation Scales for Summer (Grid Points 4 Through 17) and Winter (Grid Points 2 Through 17) Determined From the Space-Time Correlation Functions Described in Appendix A

\begin{tabular}{lcccccc}
\hline & \multicolumn{2}{c}{ Space Scale, $\mathrm{km}$} & & \multicolumn{2}{c}{ Time Scale, days } \\
\cline { 2 - 3 } \cline { 5 - 6 } \multicolumn{1}{c}{ Season } & $\tau$ & $\zeta$ & & $\tau$ & $\zeta$ \\
\hline All summers & 699 & 1085 & & 1.36 & 3.48 \\
All winters & 988 & 1324 & & 1.21 & 3.51 \\
\hline
\end{tabular}

nia Bight [Halliwell and Allen, 1987]. The methods used to calculate these functions and the integral correlation space and time scales are presented in Appendix A. The correlation space scales of $\tau$ are $699 \mathrm{~km}$ in summer and $988 \mathrm{~km}$ in winter (Table 4). The correlation time scales of $\tau$ are 1.36 days in summer and 1.21 days in winter. The $\tau$ space scale is $41 \%$ greater in winter than in summer, and the $\tau$ time scale is $11 \%$ greater in summer than in winter. The space and time scales of $\zeta$ are both substantially larger than those of $\tau$. The space scale of $\zeta$ is $1085(1324) \mathrm{km}$ in summer (winter), and the time scale of $\zeta$ is 3.48 (3.51) days in summer (winter). The $\zeta$ space scale is $22 \%$ greater in winter than in summer, but the $\zeta$ time scale is nearly equal in both seasons.

The orientation of the central correlation ridge of $\hat{R}_{\pi t}$ is determined by the propagation characteristics of the $\tau$ field (Figure 4). This ridge is symmetric about the lag time and lag distance axes in summer, and therefore no preferred propagation direction is evident. Along the major axis of the ridge in winter, the ratio of space lag to time lag is about $-2500 \mathrm{~cm}$ $\mathrm{s}^{-1}$, indicating that equatorward propagation of $\tau$ fluctuations is dominant in winter. The central correlation ridges of $\hat{R}_{\zeta \zeta}$ indicate that poleward propagation of $\zeta$ fluctuations is dominant in both summer and winter (Figure 4). The $\zeta$ ridges are insufficiently elongated to make accurate estimates of propagation speeds, but since $\zeta$ fluctuations are partly a forced re- sponse, these estimates do not represent free-wave phase speeds [Allen and Denbo, 1984].

The maxima of the cross-correlation functions $\hat{R}_{\zeta \tau}$ are 0.51 (0.55) in summer (winter), and they occur at a space lag of $-360 \mathrm{~km}$ in both seasons and at a time lag of $-1.25(-0.75)$ days in summer (winter). Thus $\zeta$ fluctuations at a given location along the coast are most highly correlated with $\tau$ fluctuations that occur equatorward and earlier in time in both summer and winter, consistent with the summer 1973 observations of Halliwell and Allen [1984]. This correlation displacement results if one coastally trapped wave mode governed by (1) dominates the $\zeta$ response [Allen and Denbo, 1984]. Freewave phase speeds of $\zeta$ can be determined from the slope of the line connecting the origin (zero space and time lags) with the peak of $\hat{\boldsymbol{R}}_{\zeta \tau}$ [Allen and Denbo. 1984], yielding estimates here of $330(230-460) \mathrm{cm} \mathrm{s}^{-1}$ in summer and $560(360-830) \mathrm{cm}$ $\mathrm{s}^{-1}$ in winter. The error ranges for these estimates are based on an uncertainty of $\pm 90 \mathrm{~km}$ in lag distance and \pm 0.125 day in lag time of the location of the $\hat{R}_{\zeta \tau}$ peak. The estimated free-wave phase speed is substantially larger in winter, but owing to the large uncertainty in these estimates, we cannot conclude that this difference is statistically significant.

The summer and winter correlation ridges of $\hat{R}_{\zeta \tau}$ also have distinctly different shapes (Figure 4). In winter, the ridge has an extension from the correlation peak toward positive space lag and negative time lag, giving the full ridge a teardrop shape. The slope of this extension is negative and approximately equals the slope of $\hat{R}_{\tau \tau}$ in winter of $-2500 \mathrm{~cm} \mathrm{~s}^{-1}$. This indicates that at least part of the winter $\zeta$ response propagates alongshore at the same speed as $\tau$ disturbances.

Coherence scales and propagation characteristics of the $\tau$ and $\zeta$ fields can be studied in the frequency domain using the space-lagged coherences squared $\hat{\gamma}_{\tau \tau}{ }^{2}\left(\eta_{n}, \omega_{l}\right), \hat{\gamma}_{\zeta \zeta}{ }^{2}\left(\eta_{n}, \omega_{l}\right)$, and $\hat{\gamma}_{\zeta \tau}{ }^{2}\left(\eta_{n}, \omega_{l}\right)$ and phase functions $\hat{\theta}_{\tau \tau}\left(\eta_{n}, \omega_{l}\right), \hat{\theta}_{\zeta \zeta}\left(\eta_{n}, \omega_{l}\right)$, and $\hat{\theta}_{\zeta \tau}\left(\eta_{n}\right.$, $\left.\omega_{1}\right)$. These functions are contoured in Figures 5 and 6 , and the methods of calculating them are presented in Appendix A. We

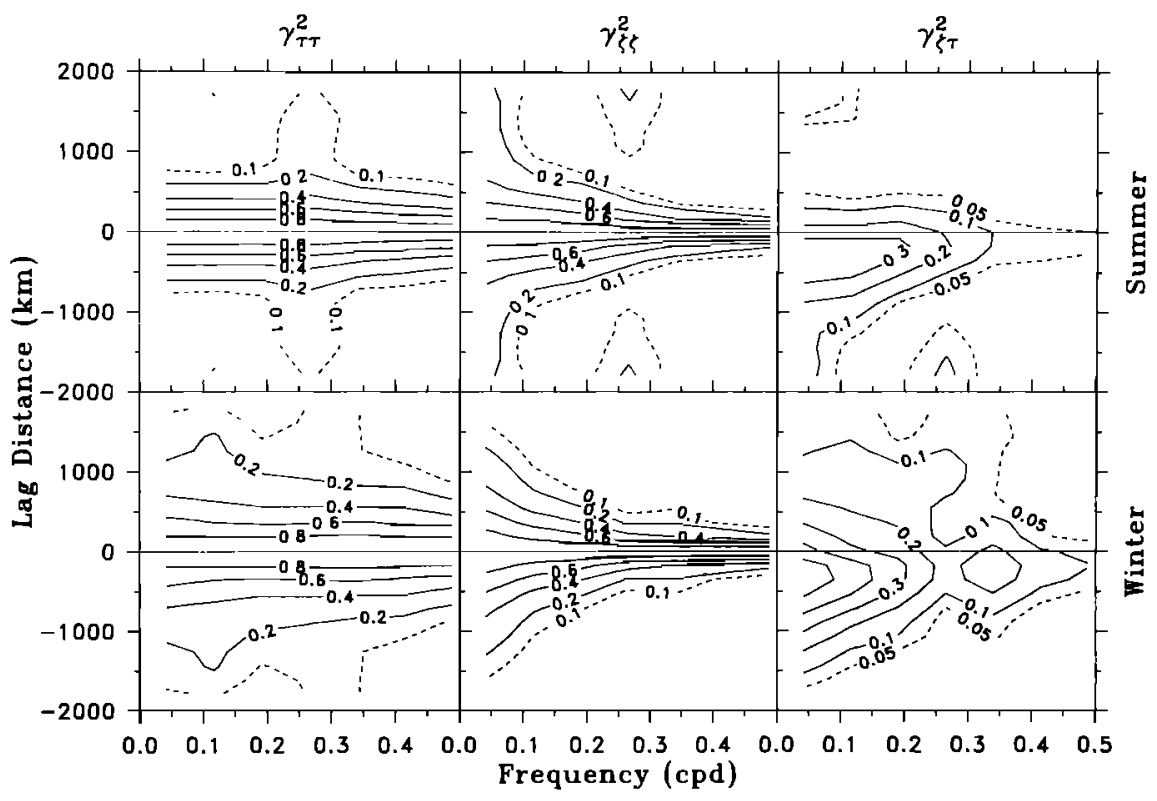

Fig. 5. The space-frequency coherence squared $\hat{\gamma}_{\mathrm{tr}}{ }^{2}\left(\eta_{n}, \omega_{l}\right), \hat{\gamma}_{\xi \zeta}{ }^{2}\left(\eta_{n}, \omega_{l}\right)$, and $\hat{\gamma}_{\zeta \tau}{ }^{2}\left(\eta_{n}, \omega_{l}\right)$, calculated from space-averaged autospectra and cross-spectra and ensemble averaged over the four summers (grid points 4 through 17) and four winters (grid points 2 through 17). A $\hat{r}^{2}$ of 0.041 is statistically significant to a $95 \%$ level of confidence (Appendix A). 


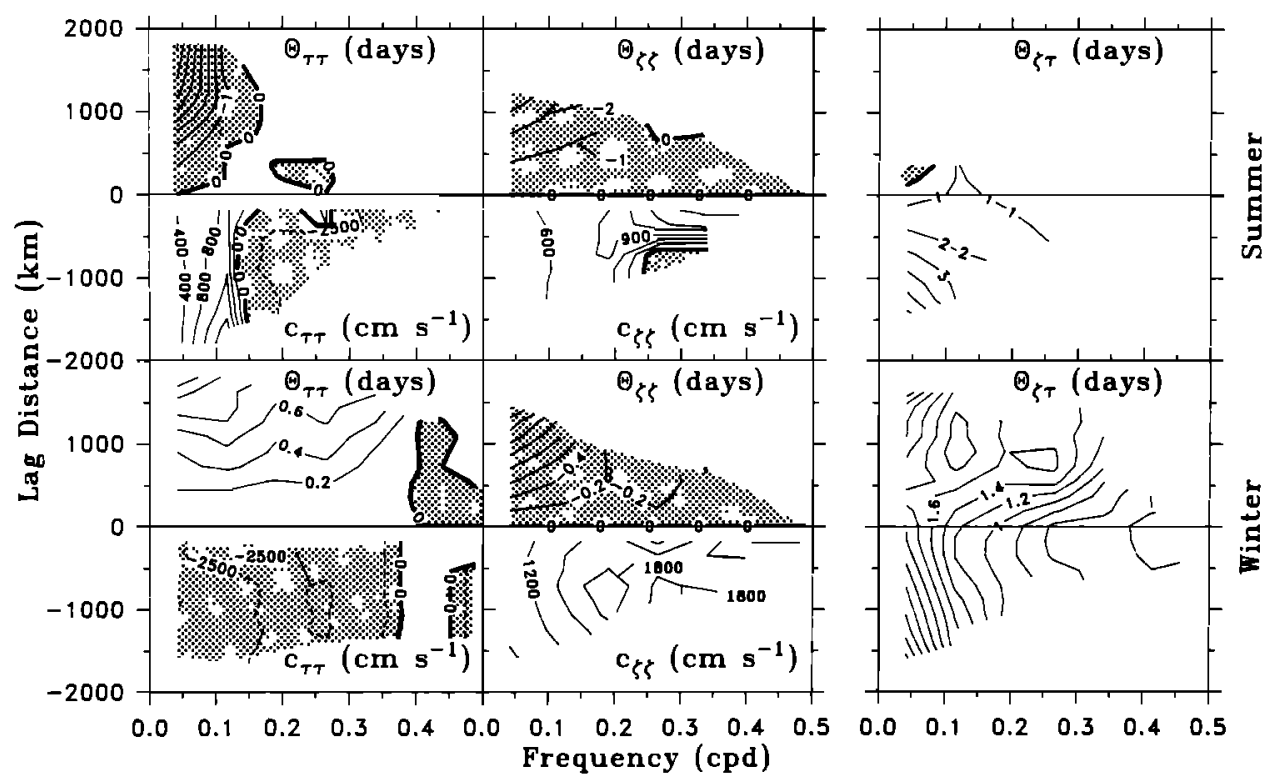

Fig. 6. The space-frequency phase lags $\hat{\theta}_{\tau \tau}\left(\eta_{n}, \omega_{l}\right), \hat{\theta}_{\xi \zeta}\left(\eta_{n}, \omega_{l}\right)$, and $\hat{\theta}_{\xi_{\tau}}\left(\eta_{n}, \omega_{l}\right)$ contoured in days and the phase speed functions $\hat{c}_{\mathrm{rr}}\left(\eta_{n}, \omega_{l}\right)$ and $\hat{c}_{\mathrm{tr}}\left(\eta_{n}, \omega_{l}\right)$ contoured in centimeters per second. Since they are symmetric, $\hat{\theta}_{\mathrm{r \tau}}$ is combined with $\hat{c}_{\mathrm{rt}}$, and $\hat{\theta}_{\zeta \zeta}$ ss combined with $\hat{c}_{5,}$, in the same panels. Negative $\hat{\theta}$ and $\hat{c}$ are shaded. The functions $\hat{\theta}$ and $\hat{c}$ are not contoured where the corresponding $\hat{\gamma}^{2}$ function (Figure 5) is insignificant.

use the alongshore separation at which $\hat{\gamma}^{2}$ decreases to its minimum significant value $(0.041$; see Appendix A) as the coherence scale. It exceeds $2000 \mathrm{~km}$ for $\tau$ at all $\omega$ in both seasons, for $\zeta$ at $\omega<0.15$ cycles per day (cpd) in both seasons, and for $\zeta$ near $\omega=0.26 \mathrm{cpd}$ in summer (Figure 5). The scale for $\zeta$ decreases to $<1000 \mathrm{~km}$ for $\omega \geq 0.34 \mathrm{cpd}$. These scales are substantially smaller for measured $\tau$ than for calculated $\tau$. Halliwell and Allen [1987] calculated $\hat{\gamma}_{v w}{ }^{2}$ for measured and calculated alongshore wind $v$ and determined that measured $v$ alongshore coherence scales were $30-50 \%$ smaller than those for calculated $v$. The same calculation made using measured and calculated $t$ (not shown) shows a similar decrease.

The width of the central coherence ridge for $\hat{\gamma}_{\tau \tau}^{2}$ is nearly constant over the entire $\omega$ band (0.041-0.48 cpd) in summer (Figure 5). If we define the coherence ridge as that part of ( $y$, $\omega)$ space where the coherence squared exceeds 0.4 , then the half-width of this ridge is about $400 \mathrm{~km}$ in summer. In winter, the half-width is larger and decreases from about $700 \mathrm{~km}$ to $500 \mathrm{~km}$ with increasing $\omega$ over the entire $\omega$ band. In contrast, the half-widths of the central coherence ridges for $\hat{\gamma}_{\zeta \zeta}{ }^{2}$ vary with $\omega$ by a large amount, decreasing from about 750 to 150 $\mathrm{km}$ with increasing $\omega$ in both seasons. A decrease such as this is qualitatively predicted by (1) for one mode [Allen and Denbo, 1984].

The maximum coherence ridges for $\zeta_{\zeta \tau}{ }^{2}$ are displaced toward negative space lag. In summer, the maximum coherence is at $\eta_{n}=-360 \mathrm{~km}$ for $\omega \leq 0.11 \mathrm{cpd}$, at $\eta_{n}=-180 \mathrm{~km}$ for $0,11<\omega<0.26 \mathrm{cpd}$, and at $\eta_{n}=0 \mathrm{~km}$ for $\omega \approx 0.34 \mathrm{cpd}$. The maximum coherence is small $(<0.1)$ for $\omega>0.34 \mathrm{cpd}$. This decrease in space lag with increasing $\omega$ is predicted by (1) [Allen and Denbo, 1984]. In winter, however, the largest coherences are observed at $\eta_{n}=-360 \mathrm{~km}$ for $\omega<0.19 \mathrm{cpd}$ and at $\eta_{n}=-180 \mathrm{~km}$ for $\omega \geq 0.19 \mathrm{cpd}$. The maximum winter coherences squared decrease to 0.1 only at the largest $\omega$ resolved $(0.48 \mathrm{cpd})$.
The propagation characteristics of the $\tau$ field, as illustrated by the phase function $\hat{\theta}_{\tau \tau}\left(\eta_{n}, \omega_{l}\right)$ and propagation speed function $\hat{c}_{\tau z}\left(\eta_{n}, \omega_{l}\right)$ (appendix A) differ substantially between summer and winter (Figure 6). Poleward propagation dominates in summer for $\omega \leq 0.11 \mathrm{cpd}$, and $\hat{c}_{\mathrm{rr}}$ decreases from $>1000 \mathrm{~cm} \mathrm{~s}^{-1}$ at $\omega=0.11 \mathrm{cpd}$ to about $400 \mathrm{~cm} \mathrm{~s}^{-1}$ at the smallest $\omega$ resolved $(0.041 \mathrm{cpd})$. In winter, however, equatorward propagation exists for $\omega \leq 0.34 \mathrm{cpd}$, with $\hat{c}_{\tau \tau}$ approximately constant at $-2500 \mathrm{~cm} \mathrm{~s}^{-1}$ (the same speed estimated from the correlation function $\hat{R}_{\mathrm{r \tau}}$ ). The winter $\tau$ fluctuations are evidently due to spatially coherent propagating disturbances (cyclones, anticyclones, and fronts) that generate $\tau$ fluctuations over a broad $\omega$ band, consistent with the results from the wind analysis of Halliwell and Allen [1987]. This does not seem to be true in summer, since $\hat{c}_{\tau \tau}$ is strongly frequencydependent.

The propagation characteristics of the $\zeta$ field differ substantially from those of the $\tau$ field in both summer and winter. Poleward propagation dominates at all $\omega$, and $\hat{c}_{\zeta \zeta}$ (not to be interpreted as a free-wave phase speed) increases with increasing $\omega$ from about $500 \mathrm{~cm} \mathrm{~s}^{-1}\left(1000 \mathrm{~cm} \mathrm{~s}^{-1}\right)$ at $\omega=0.041 \mathrm{cpd}$ to about $900 \mathrm{~cm} \mathrm{~s}^{-1}\left(1800 \mathrm{~cm} \mathrm{~s}^{-1}\right)$ near $\omega=0.19 \mathrm{cpd}$ in summer (winter). Increasing $\hat{c}_{\zeta \zeta}$ with increasing $\omega$ is predicted from (1) by Allen and Denbo [1984].

From the phase function $\hat{\theta}_{\zeta \tau}$, we can quantify the local phase lag of $\zeta$ to $\tau$ in units of time or degrees. In summer, the local time lag (Figure 6) is near 1 day for $\omega<0.26 \mathrm{cpd}$. In winter, however, the local lag time decreases substantially with increasing $\omega$, from about 2 days to $<0.5$ days over the entire $\omega$ band. It is interesting to note that the constant local time lag in summer corresponds to a local phase lag in degrees that increases with increasing $\omega$, an effect predicted by Allen and Denbo [1984] from (1). In winter, however, the decreasing local time lag with increasing $\omega$ corresponds to a local phase lag that is approximately constant near $60^{\circ}$ over all $\omega$ except 
TABLE 5. Total Variance, Percentage of Total Variance with $|l| \leq 1.1 \times 10^{-3} \mathrm{cpkm}$, and Percentage of Total Variance Due to Poleward Propagating Fluctuations for Summer (Grid Points 4 Through 17) and Winter (Grid Points 2 Through 17) Seasons

\begin{tabular}{|c|c|c|c|c|c|c|}
\hline \multirow[b]{2}{*}{ Season } & \multicolumn{2}{|c|}{ Total Variance } & \multicolumn{2}{|c|}{$|l| \leq 1.1 \times 10^{-3}, \%$} & \multicolumn{2}{|c|}{ Poleward Propagating, \% } \\
\hline & $\operatorname{dyn}^{2} \mathrm{~cm}^{-4}$ & $\mathrm{~cm}^{2}$ & $\tau$ & $\zeta$ & $\tau$ & $\zeta$ \\
\hline All Summers & 16.0 & 1252 & 93.7 & 93.5 & 52.2 & 58.2 \\
\hline All Winters & 190 & 3519 & 88.9 & 94.1 & 46.8 & 54.3 \\
\hline Summer 1980 & 3.63 & 169 & 93.2 & 94.5 & 48.5 & 59.2 \\
\hline Summer 1981 & 5.18 & 337 & 93.5 & 90.4 & 53.6 & 57.3 \\
\hline Summer 1982 & 3.94 & 286 & 94.1 & 93.9 & 55.2 & 61.9 \\
\hline Summer 1983 & 3.29 & 460 & 94.1 & 94.0 & 53.9 & 59.9 \\
\hline Winter $1980-1981$ & 54.0 & 738 & 91.1 & 93.5 & 46.6 & 55.1 \\
\hline Winter $1981-1982$ & 44.9 & 776 & 84.2 & 94.4 & 45.9 & 52.4 \\
\hline Winter $1982-1983$ & 69.0 & 1393 & 90.0 & 95.9 & 46.4 & 54.9 \\
\hline Winter 1983-1984 & 22.0 & 612 & 89.2 & 92.4 & 47.5 & 57.5 \\
\hline
\end{tabular}

more red. The $\omega$ autospectra for $\zeta$ are more red than those for $\tau$ in both seasons, which was predicted from (1) by Allen and Denbo [1984]. In winter, the autospectrum for $\zeta$ is more red for $\omega<0.15 \mathrm{cpd}$ than it is for larger $\omega$.

\subsection{Interannual Variability in the Autospectra of $\tau$ and $\zeta$}

The $(l, \omega)$ autospectra for the individual four summers (grid points 4 through 17) and four winters (grid points 2 through 17) are contoured for $\tau$ in Figure 9 and $\zeta$ in Figure 10, and propagating autospectra of both $\tau$ and $\zeta$ for the individual seasons are contoured in Figure 11. Although the summer and winter distributions of variance density for both $\tau$ and $\zeta$ appear qualitatively similar from year to year in Figures 9 and 10 , some interannual variability does exist for both seasons.

For $\tau$ in summer, the total variance over grid points 4 through 17 ranges from 3.29 (dyn $\left.\mathrm{cm}^{-2}\right)^{2}$ in summer 1983 to $5.18\left(\mathrm{dyn}^{-2}\right)^{2}$ in summer 1981 (CODE 1) (Table 5). Well over $90 \%$ of the total variance is due to fluctuations with $l \leq 1.1 \times 10^{-3} \mathrm{cpkm}$ in all four summers (Table 5). The large excess of poleward propagating variance observed for $\omega<0.1$ cpd in the ensemble-averaged propagating autospectra (Figure $7 b)$ results primarily from the large excess observed in summers 1981 and 1982 (CODE 1 and CODE 2) (Figure 11). Most of the excess poleward propagating variance at small $l$ is distributed over a larger $\omega$ band $(\omega<0.35 \mathrm{cpd})$ in summer 1983. Only $48.5 \%$ of the total variance propagates poleward in summer 1980 , while between $53.6 \%$ and $55.2 \%$ propagates poleward in the other summers. The largest excess is observed in summer 1982 (CODE 2) (Table 5).

For $\zeta$ in summer, the total variance over grid points 4 through 17 ranges from $169 \mathrm{~cm}^{2}$ in summer 1980 to $460 \mathrm{~cm}^{2}$ in summer 1983 (Table 5). The total variance does not appear to be related to either the total $\tau$ variance or the percentage of $\tau$ variance that propagates poleward. For example, the largest $\zeta$ variance and the smallest $\tau$ variance both occur in summer 1983 , and the largest percentage of poleward propagating $\tau$ variance and the second-smallest $\zeta$ variance both occur in summer 1982. The percentage of total variance due to fluctu- ations with $|l| \leq 1.1 \times 10^{-3} \mathrm{cpkm}$ ranges from 90.4 during summer 1981 (CODE 1) to 94.5 during summer 1980. The largest excess of poleward propagating $\zeta$ variance exists at the smallest $l$ resolved $\left(0.40 \times 10^{-3} \mathrm{cpkm}\right.$ in summer and 0.35 $\times 10^{-3} \mathrm{cpkm}$ in winter) and essentially for $\omega \leq 0.19 \mathrm{cpd}$ in all four years (Figure 11). The largest excess is observed during summer 1982 (CODE 2), where $61.9 \%$ of the total variance propagates poleward (Table 5). The smallest excess $(57.3 \%)$ occurs during summer 1981 (CODE 1).

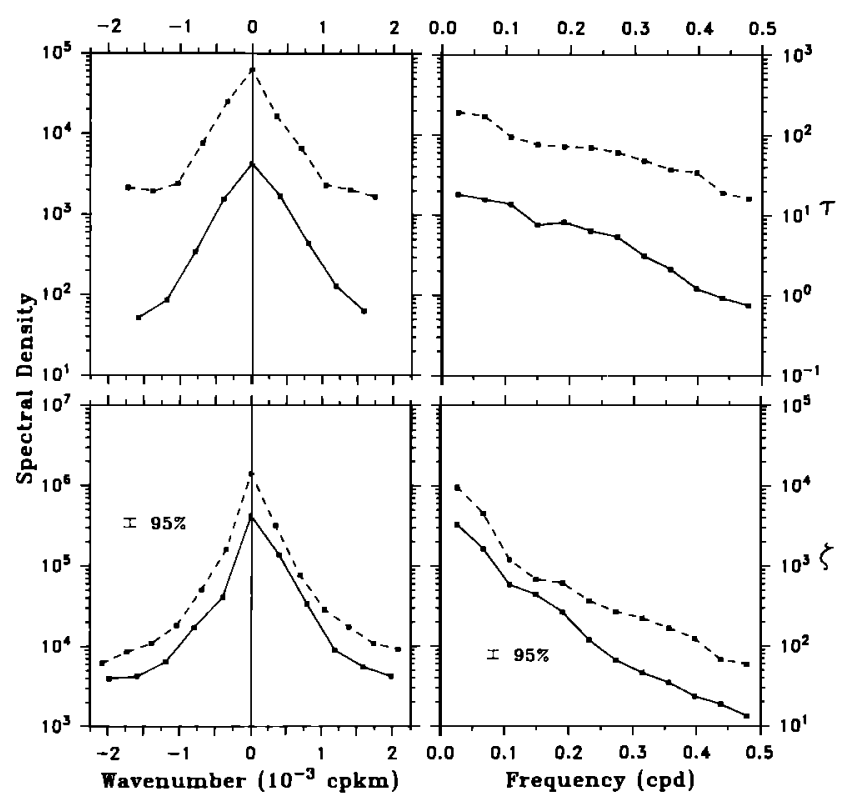

Fig. 8. (left) Integrated wave number autospectra and (right) integrated frequency autospectra for (top) $\tau$ and (bottom) $\zeta$, ensemble averaged over the four summers (grid points 4 through 17 ; solid lines) and four winters (grid points 2 through 17; dashed lines). The wave number autospectra have 480 degrees of freedom in both seasons, and the frequency autospectra have 600 (680) degrees of freedom for summer (winter). The $95 \%$ confidence band is shown. 

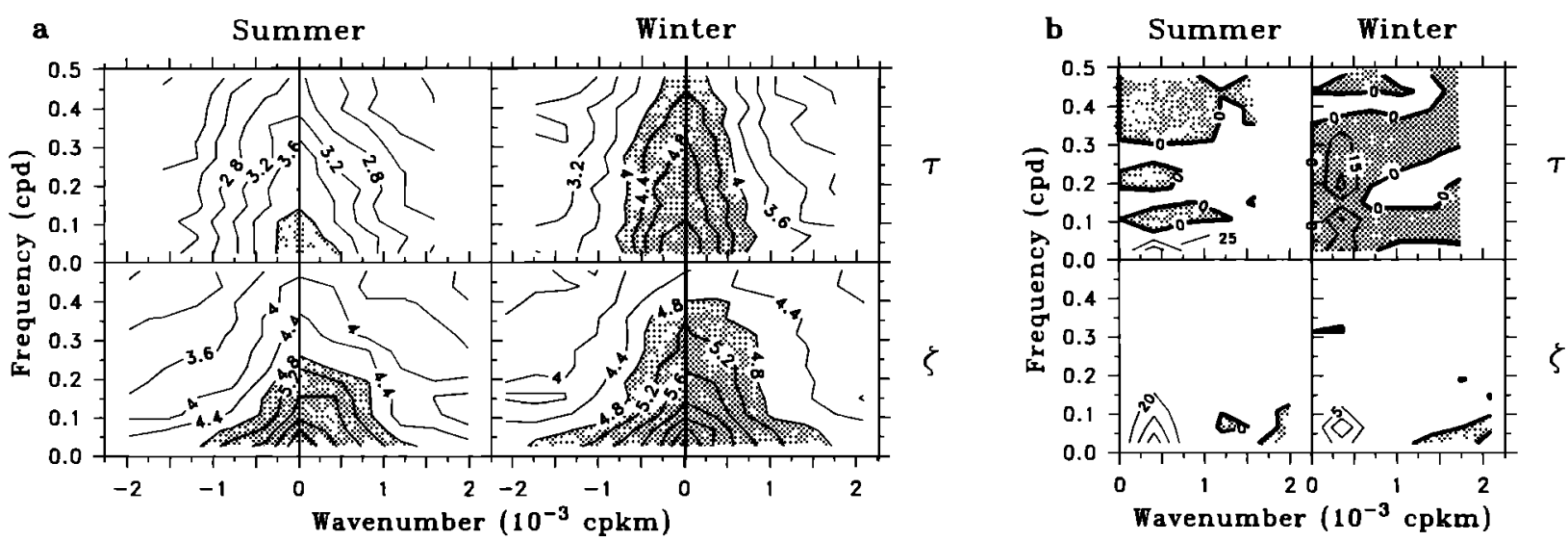

Fig. 7. (a) Wave number-frequency autospectra and $(b)$ propagating autospectra of $\tau$ and $\zeta$ ensemble averaged over the four summers (grid points 4 through 17) and four winters (grid points 2 through 17). The contours are of $\log _{10}\left[\hat{S}\left(l_{m}\right.\right.$, $\left.\left.\omega_{n}\right)\right]$ (Figure $7 a$ ) and $P\left[S\left(+l_{m}, \omega_{n}\right)-\hat{S}\left(-l_{m}, \omega_{n}\right)\right]$ (Figure $7 b$ ), with $S$ in (units) $)^{2} \mathrm{cpd}^{-1} \mathrm{cpkm}^{-1}$, where $P=10^{-2}$ for $\tau$ in summer, $P=10^{-3}$ for $\tau$ in winter, $P=10^{-4}$ for $\zeta$ in summer, and $P=10^{-5}$ for $\zeta$ in winter. Spectrum estimates have been averaged over five frequency bands, excluding $\omega_{n}=0$, and four seasons, so the number of degrees of freedom is 40 (Appendix B). The $95 \%$ confidence limits are between $\log _{10} \hat{s}-0.17$ and $\log _{10} \hat{S}+0.21$ at all $l_{m}$ and $\omega_{n}$. The autospectra in Figure $7 a$ are shaded where $\hat{S}_{\tau}>10^{4}$ and $\hat{S}_{\xi}>10^{4}{ }^{8}$. Negative propagating autospectra (equatorward propagating variance exceeds poleward propagating variance) are shaded in Figure $7 b$. The $l_{m}$ and $\omega_{n}$ at which estimates are made are shown in Figure 8.

the smallest $(0.041 \mathrm{cpd})$, where it is near $30^{\circ}$. The time lag increases with increasing equatorward space lags $\left(\eta_{n}<0 \mathrm{~km}\right)$ in both seasons, consistent with a $\zeta$ response that tends to propagate poleward.

\section{Wave Number-Frequency AutosPeCtra of $\tau$ AND $\zeta$}

\subsection{Summer and Winter Autospectra of $\tau$ and $\zeta$}

Autospectra of $\tau$ and $\zeta\left[\hat{S}_{r}\left(l_{m}, \omega_{n}\right)\right.$ and $\left.\hat{S}_{\zeta}\left(l_{m}, \omega_{n}\right)\right]$ for summer (grid points 4 through 17 ) and winter (grid points 2 through 17) (Figure 7a) are calculated using the two-dimensional Fourier transform method described in Appendix B. To correct for the possible effects of leakage in the wave number domain, we prewhitened all data sets in the alongshore domain using the first-difference method, and then postcolored all spectrum functions in the wave number domain to recover the true spectrum estimates [e.g., Frankignoul, 1974]. The motivation behind this correction is discussed in Appendix B. The spectrum estimates for positive $l$ represent poleward propagating variance, and those for negative $l$ represent equatorward propagating variance. The total variance of the $\tau$ and $\zeta$ fields in summer and winter, calculated by adding the total variances of the individual seasons, is listed in Table 5.

Most of the variance of $\tau$ and $\zeta$ exists for $|l| \leq 1.1 \times 10^{-3}$ cpkm (wavelengths of $\geq 900 \mathrm{~km}$ ) (Figure $7 a$ ). For $\tau$, the variance density decreases by about a factor of 10 for $|l|$ increasing from 0 to $1.1 \times 10^{-3} \mathrm{cpkm}$ over all frequencies resolved $(0.025-0.48 \mathrm{cpd})$ in both seasons. For $\zeta$, the corresponding decrease is greater for negative $l$ than for positive $l$ and becomes smaller for increasing $\omega$. It exceeds a factor of 10 only for $\omega<0.27(<0.15) \mathrm{cpd}$ for negative (positive) $l$ in summer, and only for $\omega<0.36(<0.15)$ cpd for negative (positive) $l$ in winter. This wave number asymmetry results from the strong tendency for $\zeta$ fluctuations to propagate poleward. The preferred propagation direction in $(l, \omega)$ space is best illustrated by contouring the propagating autospectra $[\hat{S}(+l, \omega)-\hat{S}(-l$, $\omega)]$ of $\zeta$ and $\tau$ (Figure $7 b$ ). In summer, poleward propagation dominates for $\tau$ over all $l$ for $\omega \leq 0.066 \mathrm{cpd}$, and the excess of poleward propagating variance is greatest for $l<10^{-3} \mathrm{cpkm}$. Equatorward propagating variance slightly exceeds poleward propagating variance at higher frequencies. In winter, equatorward propagation dominates for $\tau$ in most of $(l, \omega)$ space, with the largest excess of variance existing for $l<0.6 \times 10^{-3} \mathrm{cpkm}$ and $\omega<0.36 \mathrm{cpd}$. In contrast to $\tau$, poleward propagation dominates $\zeta$ fluctuations in both summer and winter at nearly all $l$ and $\omega$, with the greatest excess existing for $l \leq 1.1 \times 10^{-3}$ cpkm and $\omega \leq 0.19 \mathrm{cpd}$ in summer and $\leq 0.15 \mathrm{cpd}$ in winter. The largest excess of poleward propagating $\zeta$ variance for both seasons is found at the smallest $l$ resolved $\left(0.40 \times 10^{-3}\right.$ cpkm in summer and $0.35 \times 10^{-3} \mathrm{cpkm}$ in winter), and at $\omega=0.025 \mathrm{cpd}$ in summer and $0.066 \mathrm{cpd}$ in winter.

The overall distributions of variance density as functions of $l$ and $\omega$ separately are illustrated by integrating $\hat{S}_{q}\left(l_{m}, \omega_{n}\right)$ and $\hat{S}_{\xi}\left(l_{m}, \omega_{n}\right)$ over $\omega_{n}$ and $l_{m}$, respectively. It is evident from the resulting $l$ and $\omega$ autospectra (Figure 8) that the increase of $\tau$ variance in winter over summer is much greater than the corresponding increase of $\zeta$ variance (Table 5). We noted earlier that this winter increase is a function of $y$ (section 3.1, Figure 3).

The $l$ autospectra illustrate the dominance of large wavelengths for the $\tau$ and $\zeta$ fluctuations. In summer, $93.7 \%(93.5 \%)$ of the $\tau(\zeta)$ variance is at $|l| \leq 1.1 \times 10^{-3} \mathrm{cpkm}$ (Table 5). In winter, this percentage of $\tau$ variance decreases to 88.9, while the percentage of $\zeta$ variance is 94.1 . The asymmetry of the $l$ autospectra illustrates the preferred propagation direction of the fluctuations. In summer, $52.2 \%(58.2 \%)$ of the total $\tau(\zeta)$ variance is due to poleward propagating fluctuations (Table 5). In winter, this percentage of $\tau$ variance decreases to 46.8 , and this percentage of $\zeta$ variance decreases to 54.3. The $\omega$ autospectra for $\tau$ decrease monotonically with increasing $\omega$ in both seasons, with the summer autospectrum being slightly 


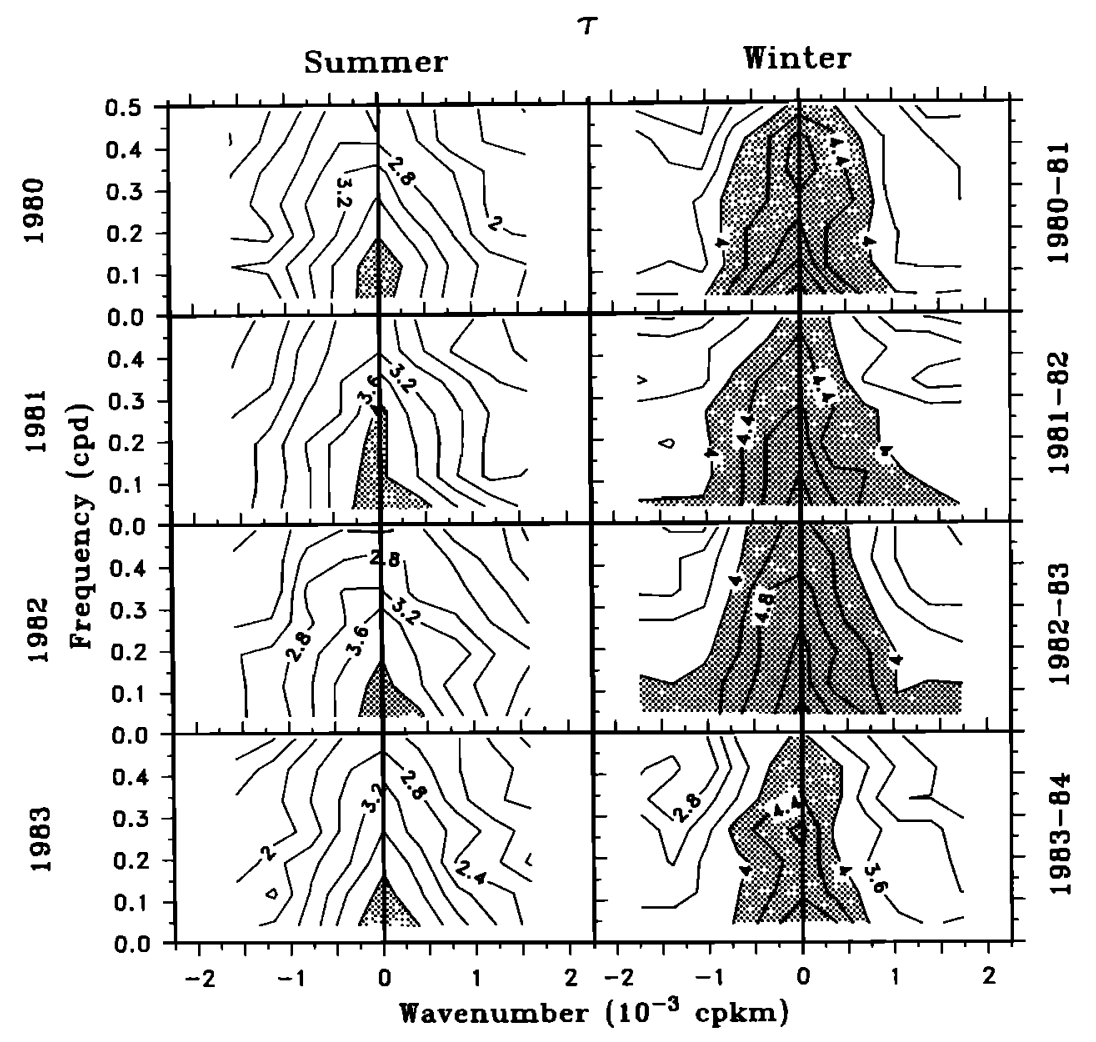

Fig. 9. Wave number-frequency autospectra of $\tau$ for the individual four summers (grid points 4 through 17) and four winters (grid points 2 through 17). The contours are of $\log _{10}\left[\hat{S}\left(l_{\mathrm{m}}, \omega_{n}\right)\right]$, with $\hat{S}$ in $\left(\mathrm{dyn} \mathrm{cm} \mathrm{cm}^{-2}\right)^{2} \mathrm{cpd}^{-1} \mathrm{cpkm}^{-1}$. Spectrum estimates have been averaged over nine frequency bands, excluding $\omega_{n}=0$, so the number of degrees of freedom is 18 . The $95 \%$ confidence limits are between $\log _{10} \hat{S}-0.24$ and $\log _{10} \hat{S}+0.34$ at all $l_{m}$ and $\omega_{n}$. The autospectra are shaded where $\hat{S}\left(l_{m}, \omega_{n}\right)>10^{4}$. The $I_{m}\left(\omega_{n}\right)$ at which estimates are made are shown in Figure 8 (Figure 13).

For $\tau$ in winter, the total variance over grid points 2 through 17 ranges from 22.0 (dyn $\left.\mathrm{cm}^{-2}\right)^{2}$ in winter 1983-1984 to $69.0\left(\mathrm{dyn}^{-2}\right)^{2}$ in winter $1982-1983$. The percentage of total variance due to fluctuations with $|l| \leq 1.1 \times 10^{-3} \mathrm{cpkm}$ ranges from 84.2 during winter 1981-1982 to 91.1 during winter 1980-1981. Equatorward propagating variance exceeds poleward propagating variance in all four winters, and the excess is largest for the smallest nonzero $|l|$ resolved. The frequency bands where the excess is greatest shift substantially from year to year. During winter 1980-1981, a small excess of poleward propagating variance exists for $\omega>0.25 \mathrm{cpd}$. During winters 1982-1983 and 1983-1984, small excesses of poleward propagating variance existed only at the smallest $\omega$ resolved $(0.041 \mathrm{cpd})$. The percentage of total variance that propagates poleward ranges from 45.9 in winter 1981-1982 to 47.5 in winter $1983-1984$ (Table 5).

For $\zeta$ in winter, the total variance over grid points 2 through 17 ranges from $612 \mathrm{~cm}^{2}$ in winter $1983-1984$ to 1393 $\mathrm{cm}^{2}$ in winter 1982-1983. These are the same winters when the $\tau$ variance is smallest and largest, respectively. The total variance does not appear to be related to the percentage of $\tau$ variance that propagates poleward. The percentage of total variance due to fluctuations with $|l| \leq 1.1 \times 10^{-3} \mathrm{cpkm}$ ranges from 92.4 in winter $1983-1984$ to 95.9 in winter $1982-1983$. The excess of poleward propagating variance is largest at the smallest nonzero $|l|$ resolved and spans all or nearly all of the entire frequency band resolved in all winters except 19811982, where equatorward propagation dominates for $\omega>0.2$ cpd. This indicates that a substantial part of the $\zeta$ response propagated alongshore along with the $\tau$ disturbances during this winter, which also had the largest percentage excess of equatorward propagating $\tau$ variance. The percentage of total variance that propagates poleward ranges from 52.4 in winter $1981-1982$ to 57.5 in winter $1983-84$ (Table 5).

\subsection{Comparison of Measured and Calculated $\tau$ Autospectra}

We test the reliability of calculated $\tau$ autospectra by comparing them to measured $\tau$ autospectra for summers 1981 and 1982, plus winter 1981-1982 (Figure 12), calculated over grid points 4 through 13 only. Since calculated and measured $\tau$ have substantially different variance at many locations along the coast, it is important to assess how much this problem will contribute to observed differences between calculated and measured $\tau$ autospectra. We therefore also compare autospectra of measured $\tau$ to autospectra of adjusted measured $\tau$ (section 2).

Similar distributions of variance density in $(l, \omega)$ space are observed for both measured and adjusted measured $\tau$ (Figure 12). If the differences in total variance are taken into account, then based on the statistical $F$ test as described by Halliwell 


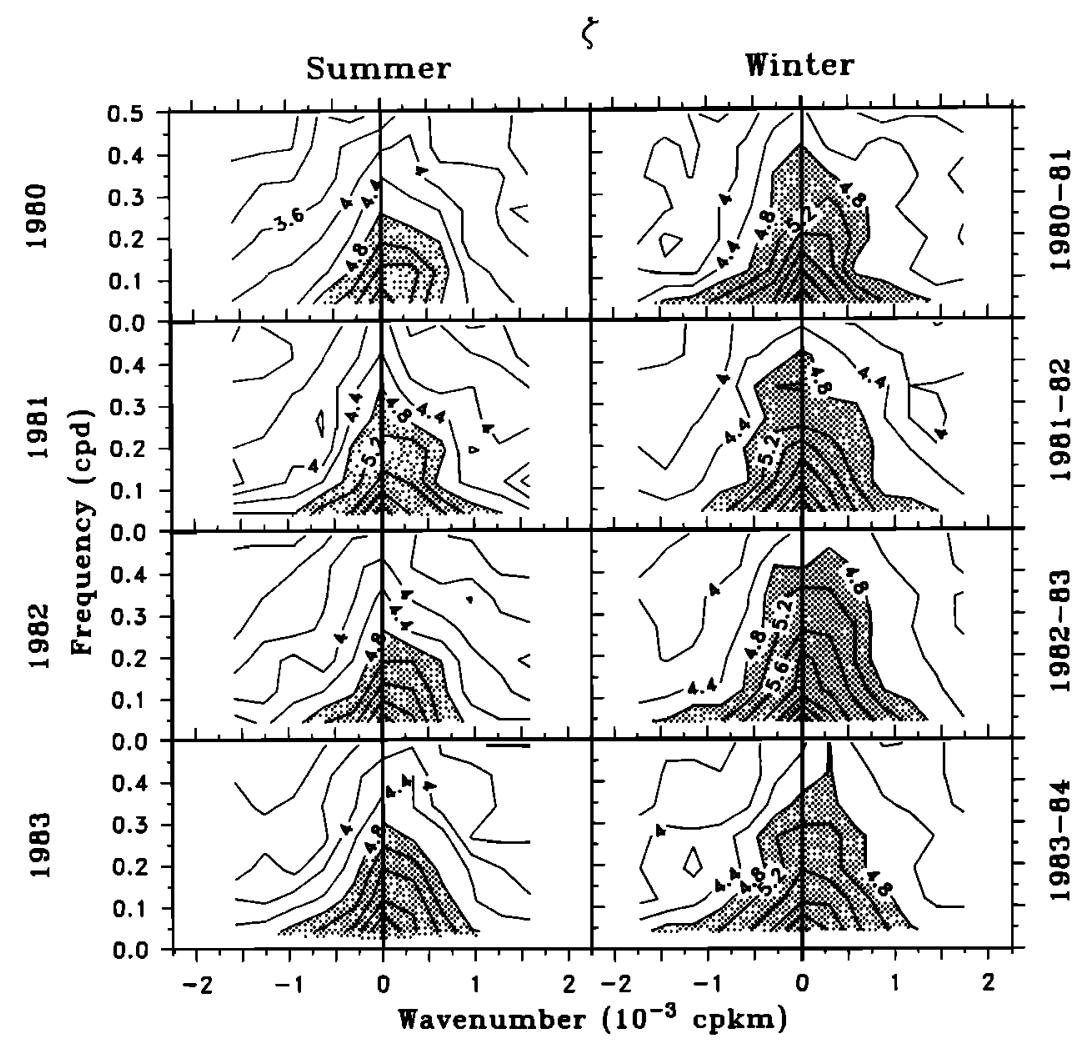

Fig. 10. Wavenumber-[requency autospectra of $\zeta$ for the individual four summers (grid points 4 through 17) and four winters (grid points 2 through 17). The contours are of $\log _{10}\left[\hat{S}\left(l_{m}, \omega_{n}\right)\right]$, with $\hat{S}$ in $\mathrm{cm}^{2} \mathrm{cpd}^{-1} \mathrm{cpkm}^{-1}$. The number of degrees of freedom is 18 (see Figure 9). The $95 \%$ confidence limits are between $\log _{10} \hat{S}-0.24$ and $\log _{10} \hat{S}+0.34$ at all $l_{m}$ and $\omega_{n}$. The autospectra are shaded where $\hat{S}\left(l_{m}, \omega_{n}\right)>10 .{ }^{4}$. The $l_{m}\left(\omega_{n}\right)$ at which estimates are made are shown in Figure 8 (Figure 13).

and Allen [1987], adjusted measured $\tau$ spectrum estimates do not differ significantly (to $95 \%$ confidence) from those of measured $\tau$ throughout $(l, \omega)$ space in all three seasons, except for $l<-1.5 \times 10^{-3}$ and $\omega>0.35 \mathrm{cpd}$ in winter 1981-1982. The similarities in the distributions of variance density are evident by inspecting integrated $l$ and $\omega$ autospectra (Figure 13). The variance of measured and calculated $\tau$ differs by an order of magnitude at some locations along the coast, yet the shape of the $(l, \omega)$ autospectra of measured $\tau$ are not significantly distorted when the variance of this field is adjusted to equal that of calculated $\tau$. Uncertainties in the representation of $\tau$ variance by the calculated and measured $\tau$ fields therefore do not significantly influence the results of our $(l, \omega)$ autospectrum analyses. We can compare calculated $\tau$ autospectra directly to those of either measured or adjusted measured $\tau$ to characterize similarities and differences between $(l, \omega)$ autospectra of the calculated and measured $\tau$ fields.

Significant differences do exist between the distributions of calculated and measured $\tau$ variance densities in $(l, \omega)$ space. Comparing the calculated and adjusted measured $\tau$ autospectra in Figure 12, calculated $\tau$ variance density decreases much more rapidly with increasing $|l|$ than it does for measured $\tau$. This difference is observed more clearly in the integrated $l$ autospectra in Figure 13. The adjusted measured $\tau$ spectrum estimates are smaller than the calculated $\tau$ estimates at $l=0$ in all seasons, but more so in both summers. These estimates are about equal at the smallest nonzero $|l|$ resolved $\left(0.55 \times 10^{-3} \mathrm{cpkm}\right)$ in all seasons, but for larger $|l|$ the estimates for calculated $\tau$ decrease much more rapidly with increasing $|l|$ than those for adjusted measured $\tau$. For $|l|=2.2$ $\times 10^{-3}$, the differences are about a factor of 5 in summer 1981 , a factor of 3.5 in winter 1981-1982, and a factor of 10 in summer 1982 . Typically, between $75 \%$ and $80 \%$ of measured and adjusted measured $\tau$ variance, and over $90 \%$ of calculated $\tau$ variance, is due to fluctuations with $|l| \leq 1.1 \times 10^{-3} \mathrm{cpkm}$ (Table 6). In contrast, the distributions of variance density as a function of $\omega$ are very similar for both calculated and adjusted measured $\tau$ (Figure 13). Comparing the $(l, \omega)$ autospectra of calculated and adjusted measured $\tau$ using the $F$ test indicates that for all three seasons, the spectrum estimates are not significantly different (to $95 \%$ confidence) for $|l| \leq 1.1 \times \mathbf{1 0}^{-\mathbf{3}}$ cpkm within the frequency band $\omega<0.34 \mathrm{cpd}$, and for $|l| \leq$ $0.55 \times 10^{-3} \mathrm{cpkm}$ within the frequency band $0.34 \leq \omega \leq 0.48$ cpd.

The calculated $\tau$ field underestimates the magnitude of poleward-propagating $\tau$ variance in summer, more so in summer 1982 than summer 1981 (Table 6). The probable cause is the relatively poor representation of wind fluctuations caused by coastally trapped, poleward propagating atmospheric pressure disturbances along the California coast 


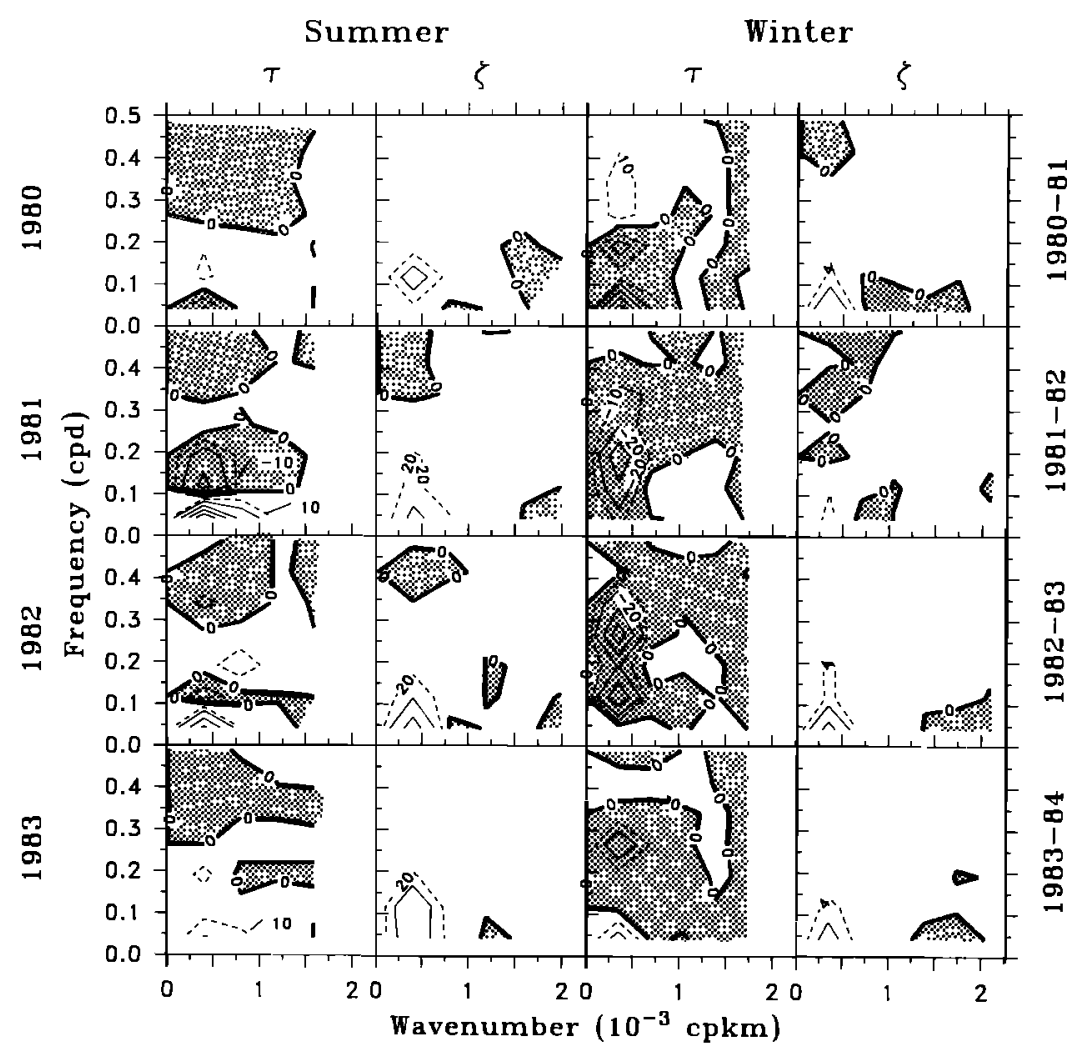

Fig 11. Wave number-[requency propagating autospectra of $\tau$ and $\zeta$ for the individual four summers (grid points 4 through 17) and four winters (grid points 2 through 17). The contours are of $P\left[\hat{S}\left(+l_{m}, \omega_{n}\right)-\hat{S}\left(-l_{m}, \omega_{n}\right)\right]$ (bottom), with $\hat{S}$ in (units) ${ }^{2} \mathrm{cpd}^{-1} \mathrm{cpkm}^{-1}$, where $P=10^{-2}$ for $\tau$ in summer, $P=10^{-3}$ for $\tau$ in winter, $P=10^{-4}$ for $\zeta$ in summer, and $P=10^{-5}$ for $\zeta$ in winter. The number of degrees of freedom is 18 (see Figure 9). Negative propagating autospectra (equatorward propagating variance exceeds poleward propagating variance) are shaded. The $l_{m}\left(\omega_{n}\right)$ at which estimates are made are shown in Figure 8 (Figure 13).

[Dorman, 1985, 1987] by the calculated wind field, as documented by Halliwell and Allen [1987]. These disturbances were more common in summer 1982 than in summer 1981.

Both adjusted measured and measured $\tau$ are very coherent with calculated $\tau$ at small $|l|$ for the three seasons (Figure 14), with coherences squared $\geq 0.6$ observed in that part of $(l, \omega)$ space where the corresponding autospectra are large. (The method of calculating these coherence functions is presented in Appendix B.) Similar coherence patterns with calculated $\tau$ are observed for both measured and adjusted measured $\tau$, with slightly larger coherence observed for adjusted measured $\tau$ in both summer 1981 and winter 1981-1982, and for measured $\tau$ in summer 1982. Consequently, the coherence between the calculated and measured $\tau$ fields is not significantly influenced by large differences in the alongshore distribution of measured and calculated $\tau$ variance. Coherence is larger for positive $l$ in summer and for negative $l$ in winter, so it tends to be largest at those $l$ and $\omega$ where $\tau$ variance is largest. On the basis of these autospectrum and cross-spectrum analyses, we conclude that the calculated $\tau$ field represents fluctuations with $|l| \leq 1.1$ $\times 10^{-3} \mathrm{cpkm}$ with reasonable accuracy for the purpose of $(l, \omega)$ autospectrum analysis.

\section{Observed $\zeta$ Response Properties in $(l, \omega)$ Space}

\subsection{Summer and Winter Response of $\zeta$ to $\tau$}

Properties of the response of $\zeta$ to $\tau$ in $(l, \omega)$ space are illustrated by the coherence squared between $\tau$ and $\zeta\left[\hat{\gamma}_{z \zeta}{ }^{2}\left(l_{m}, \omega_{n}\right)\right]$, and by the gain and phase of the transfer function of the $\zeta$ response to $\tau\left[\hat{G}_{\tau \zeta}\left(l_{m}, \omega_{n}\right)\right.$ and $\left.\hat{\theta}_{\tau \zeta}\left(l_{m}, \omega_{n}\right)\right]$ (Figure 15). (The methods of calculating these functions are described in Appendix B.) In both seasons, relatively large coherence is observed only for $|l| \leq 1.1 \times 10^{-3} \mathrm{cpkm}$ (Figure 15). In summer, $\hat{\gamma}_{\tau \zeta}^{2}$ is relatively large for positive $l$ and $\omega<0.3 \mathrm{cpd}$, while in winter, it is relatively large for negative $l$ and $\omega<0.4 \mathrm{cpd}$. The shift of large coherence toward negative $l$ in winter likely occurs because equatorward propagation dominates the $\tau$ fluctuations.

In contrast, the gain of the $\zeta$ response tends to be larger for positive $l$ in both seasons (Figure 15). The largest gain is observed for the smallest positive $l$ resolved in both seasons $\left(0.40 \times 10^{-3} \mathrm{cpkm}\right.$ in summer and $0.35 \times 10^{-3} \mathrm{cpkm}$ in winter), but at the lowest frequency resolved $(0.025 \mathrm{cpd})$ in summer and at the second-lowest frequency resolved $(0.066$ cpd) in winter. These are the same values of $l$ and $\omega$ where the largest excesses of poleward propagating $\zeta$ variance are observed (Figure $7 \mathrm{~b}$ ). A ridge of relatively large gain extends approximately along a line of constant $\omega / l$, corresponding to $\hat{c} \approx 300 \mathrm{~cm} \mathrm{~s}^{-1}$. The gain decreases along this ridge for increasing $l$ and $\omega$ to $<50 \%$ of the maximum at approximately $\omega=0.25 \mathrm{cpd}$ and $l=0.75 \times 10^{-3} \mathrm{cpkm}$ in summer. It decreases more rapidly with increasing $l$ and $\omega$ in winter, causing the largest gain to be concentrated at smaller $l$ and $\omega$ than it is in summer. The gain is substantially larger in summer than in winter. For a given total variance of $\tau$ fluctuations, the winter $\zeta$ response will be smaller than in summer for two reasons: the smaller gain throughout $(l, \omega)$ space, and the dominance of 


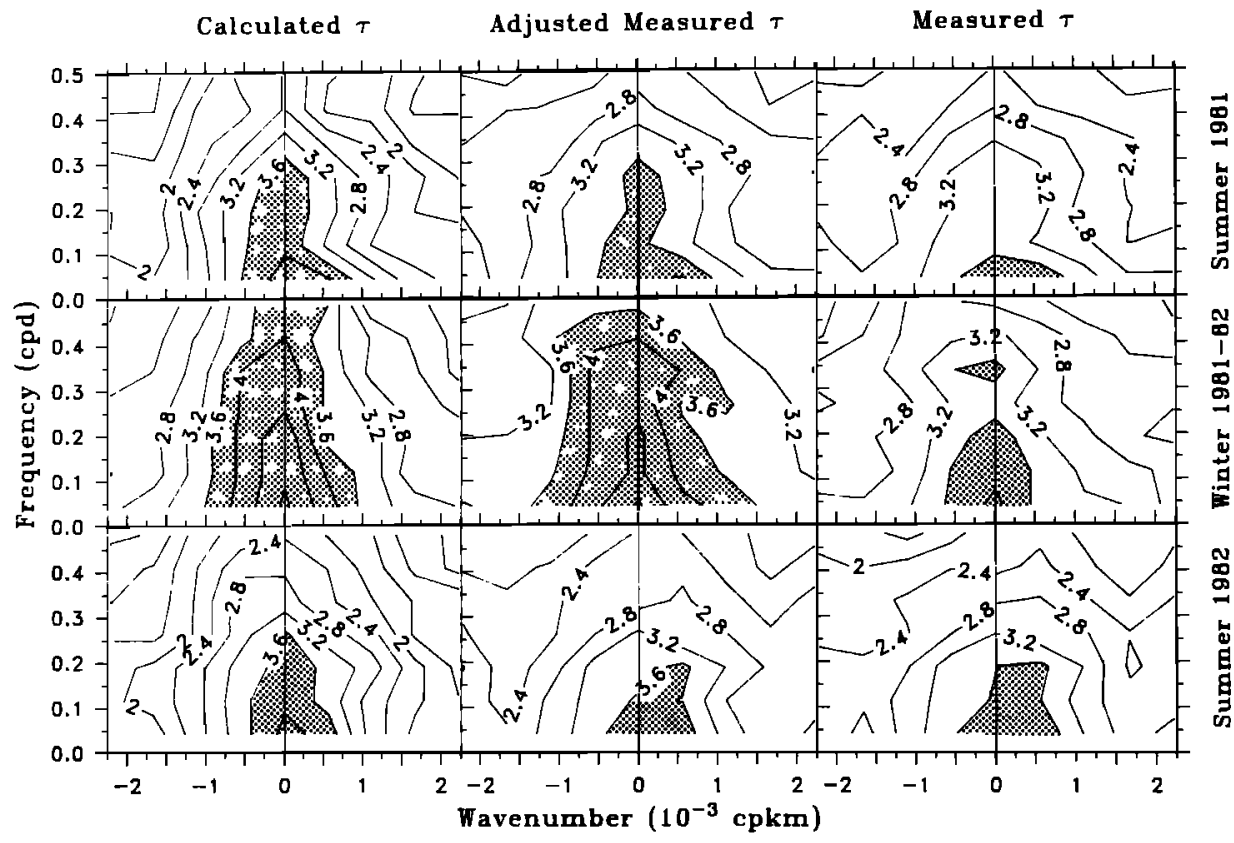

Fig. 12. Wave number-frequency autospectra of calculated, adjusted measured, and measured $\tau$ at grid points 4 through 13 for two summers and one winter. The contours are of $\log _{10}\left[S\left(l_{m}, \omega_{n}\right)\right]$, with $\hat{S}$ in units of $\mathrm{m}^{2} \mathrm{~s}^{-2} \mathrm{cpd}^{-1}$ $\mathrm{cpkm}^{-1}$. Spectrum estimates have been averaged over nine frequency bands, excluding $\omega_{n}=0$, so the number of degrees of freedom is 18 . The $95 \%$ confidence limits are between $\log _{10} \hat{S}-0.24$ and $\log _{10} \hat{S}+0.34$ at all $l_{m}$ and $\omega_{n}$. The autospectra are shaded where $\hat{S}\left(l_{m}, \omega_{n}\right)>10^{36}$. The $l_{m}$ and $\omega_{n}$ at which estimates are made are shown in Figure 13 .

equatorward propagation in the $\tau$ fluctuations, which causes $\tau$ variance in winter to exist farther from the ridge of large gain in $(l, \omega)$ space.

The corresponding phase functions for both summer and winter change sign on either side of an approximately straight line that roughly follows the ridges of maximum gain in $(l, \omega)$ space, so the $\zeta$ response is nearly in phase with $\tau$ at those $l$ and $\omega$ where the largest gain is observed. The zero-phase contour is not as well defined in winter as it is in summer, but this is largely because the winter coherence is small in that part of $(l$ $\omega)$ space. The largest possible response for a given $\tau$ disturbance will therefore occur if that disturbance propagates alongshore at speed $\hat{c}=\omega / l$ as defined by the ridge of large gain, and this response will be nearly in phase with the forcing. Most $\tau$ and $\zeta$ variance is found in that part of $(l, \omega)$ space where the phase is negative, so that the $\zeta$ response at a given $y$ will lag the $\tau$ forcing in time.

Integrated $l$ and $\omega$ coherence squared, gain, and phase functions (Figure 16) are calculated from the $(l, \omega)$ autospectra and cross-spectra that have been integrated over $\omega$ and $l$, respectively. The integrated $l$ coherence is larger for positive $l$ in summer and negative $l$ in winter and is relatively small for $|l|>1.1 \times 10^{-3} \mathrm{cpkm}$. In contrast, the gain is larger for positive $l$ in both seasons, relatively more so in summer, and is substantially larger for all $l$ in summer. For $l \leq 0$, the phase generally ranges between $-45^{\circ}$ and $-90^{\circ}$ ( $\zeta$ lags $\tau$ ) in both seasons. As $l$ increases from 0 , the phase increases rapidly to exceed $0^{\circ}(\zeta$ leads $\tau$ ) in summer and to appoximately equal 0 in winter. The integrated $\omega$ coherence tends to decrease with increasing $\omega$ (Figure 16). The strong bias toward small $\omega$ in the $\zeta$ response is evident in the gain for both seasons. In summer it decreases rapidly with $\omega$ to $0.35 \mathrm{cpd}$ and then is approximately constant for larger $\omega$. In winter it decreases rapidly with $\omega$ to $0.23 \mathrm{cpd}$ and then is approximately constant for larger $\omega$. The phase for both seasons is generally negative over all $\omega$, tends to decrease with increasing $\omega$ to $0.19 \mathrm{cpd}$, and is roughly constant for larger $\omega$. In summer it is near $0^{\circ}$ at the smallest $\omega$ resolved $(0.025 \mathrm{cpd})$.

\subsection{Interannual Variability in the Response of $\zeta$ to $\tau$}

The coherence squared between $\tau$ and $\zeta$, and the gain and phase of the $\zeta$ response to $\tau$, are contoured in Figures 17 through 19 for the eight individual seasons. While there are some differences in the details of the coherence patterns from year to year, the fundamental seasonal differences between the ensemble-averaged summer and winter patterns observed in Figure 15 are also observed for the individual seasons in Figure 17. The largest summer coherence is observed for positive $l$ in all summers except for 1981 (CODE 1). The largest winter coherence is observed for negative $l$, but much less so during winter 1980-1981 than during the other winters.

The gain functions (Figure 18) show substantial year-toyear variability, while the phase functions (Figure 19) generally do not. In particular, the rate of decrease of maximum gain with increasing $l$ and $\omega$ differs among the individual seasons. During summer 1980, the gain first increases with increasing $l$ and $\omega$ following the ridge, and then decreases. The gain generally decreases along the ridge in the other three 

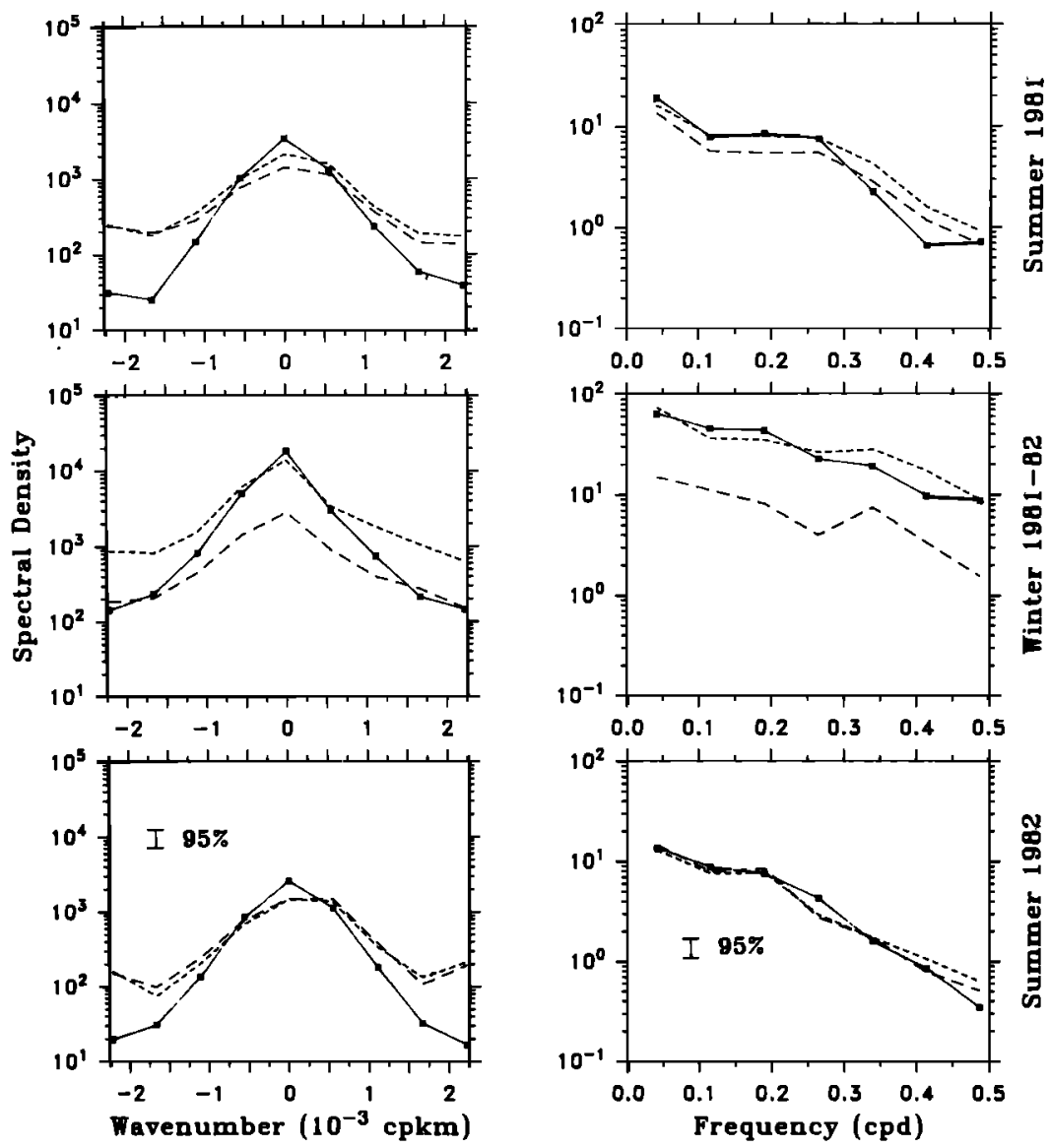

Fig. 13. (left) Integrated wave number autospectra and (right) integrated frequency autospectra for calculated $\tau$ (solid lines), adjusted measured $\tau$ (short-dashed lines), and measured $\tau$ (long-dashed lines) at grid points 4 through 13 for two summers and one winter. The integrated wave number (frequency) autospectra have 126 (198) degrees of freedom. The $95 \%$ confidence bands are shown.

summers, with the largest decrease observed during summer 1983. The decrease is smaller during winter 1980-1981 than in the other winters. The change in the sign of the phase near the dispersion curve is present in all eight seasons, although it is poorly detected during the winters owing to poor coherence in that part of $(l, \omega)$ space.

\subsection{The Response of $\zeta$ to Measured and \\ Calculated $\tau$}

We test if the observed response properties are significantly biased in any manner because we use calculated $\tau$ as the forcing function. We compare the $\zeta$ response to calculated $\tau$ with the $\zeta$ response to both measured and adjusted measured $\tau$ for three seasons, which also allows us to determine if differences in the alongshore distribution of $\tau$ variance influences the observed response properties. The coherence squared, gain, and phase functions are computed from autospectrum and crossspectrum functions for grid points 4 through 13 . These functions are contoured for summer 1981, winter 1981-1982, and summer 1982 in Figures 20 through 22, respectively.

The coherence squared, gain, and phase functions for the $\zeta$ response to measured $\tau$ are very similar to those for the $\zeta$ response to adjusted measured $\tau$, the only notable exception being differences in the magnitude of the gain function, especially in winter 1981-1982, where it is substantially larger for measured $\tau$. The large differences in the alongshore distri- bution of variance in the measured and calculated $\tau$ fields only slightly influence the observed $(l, \omega)$-dependent properties of the $\zeta$ response. The uncertainty about which $\tau$ field best represents $\tau$ variance along the coast affects only our ability to determine the magnitude of the gain, not our ability to determine qualitatively the dependence of gain and other response properties on $l$ and $\omega$. In the subsequent discussion we therefore compare the response of $\zeta$ to both calculated and measured $\tau$ without considering adjusted measured $\tau$.

TABLE 6. Percentage of Total Variance With Wavelengths $\geq 900$ $\mathrm{km}$ and Percentage of Total Variance Due to Poleward Propagating Fluctuations for Calculated, Adjusted Measured, and Measured I Over Grid Points 4 Through 13 for Summers 1981 and 1982 Plus Winter 1981-1982

\begin{tabular}{llcc}
\hline Season & Wind Stress Set & $\begin{array}{c}l^{-1} \geq 900 \\
\text { km, \%" }\end{array}$ & $\begin{array}{c}\text { Poleward } \\
\text { Propagating, \% }\end{array}$ \\
\hline Summer 1981 & calculated & 91.6 & 54.9 \\
& adjusted measured & 78.1 & 57.5 \\
Winter 1981-1982 & measured & 74.3 & 55.5 \\
& calculated & 90.9 & 48.0 \\
& adjusted measured & 78.0 & 47.2 \\
Summer 1982 & measured & 80.2 & 46.5 \\
& calculated & 93.6 & 53.6 \\
& adjusted measured & 76.9 & 58.8 \\
& measured & 78.0 & 59.9 \\
\hline
\end{tabular}




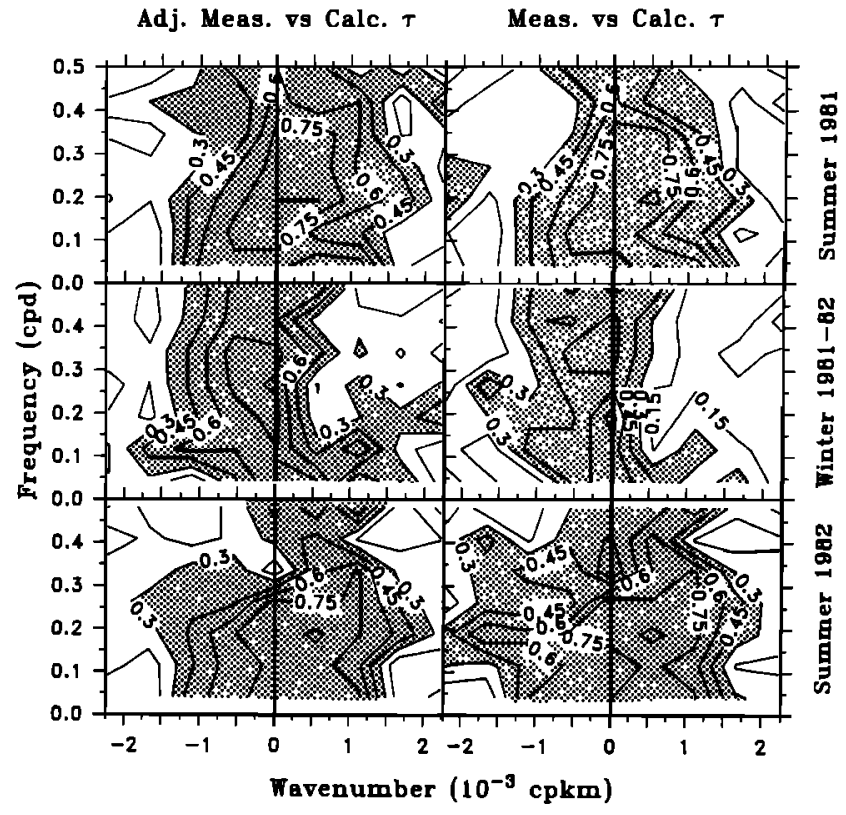

Fig. 14. Wave number-frequency coherence squared between adjusted measured and calculated $\tau$, and between measured and calculated $\tau$, at grid points 4 through 13 for two summers and one winter. The number of degrees of freedom is 18 (see Figure 12). Squared coherences of greater than 0.16 are significant to a $95 \%$ level of confidence. Squared coherences of greater than 0.3 are shaded. The $l_{m}$ and $\omega_{n}$ at which estimates are made are shown in Figure 13.

The coherences squared between $\zeta$ and both calculated and measured $\tau$ have similar patterns in $(l, \omega)$ space for all three seasons, but coherence with measured $\tau$ is somewhat larger (Figures 20 through 22). Although the coherence squared is generally statistically significant ( $>0.16$ to $95 \%$ confidence) in most of $(l, \omega)$ space, it is relatively low for $|l|>1.1 \times 10^{-3}$ cpkm, and little increase occurs at these $|l|$ when measured $\tau$ is used instead of calculated $\tau$, even though measured $\tau$ is expected to represent $\tau$ fluctuations at these $|l|$ much more accurately. The wind-driven response of $\zeta$ is apparantly weak at larger $|l|$ and can at best be marginally detected.

The patterns of the gain of the $\zeta$ response to both calculated and measured $\tau$ are similar for each season, with the tendency for a ridge of large gain to exist on the positive $l$ side of the spectrum (Figures 20 through 22). The decrease in gain along the ridge for increasing $l$ and $\omega$ exists whether calculated or measured $\tau$ is used as the forcing function, and the decrease tends to be larger for measured wind. This decrease is therefore not an artifact of using calculated $\tau$ as the forcing function, and if anything, the decreases observed for both summer and winter (Figure 15) are underestimated by using calculated $\tau$. The phase functions for calculated and measured $\tau$ also have qualitatively similar patterns in each season. We conclude that the results of our analyses have not been seriously distorted by using calculated $\tau$ to represent the forcing.

\section{Predicted $\zeta$ Response Properties in $(l, \omega)$ Space}

\subsection{Response Properties Predicted by (1) for One Mode}

The fundamental properties of the $\zeta$ response to $\tau$ in $(l, \omega)$ space predicted from basic coastally trapped wave theory can be studied using a transfer function derived by Fourier transformation of (1) in two dimensions, assuming constant parameters along the coast (Appendix $\mathrm{C}$ ). To illustrate the predicted response properties for one mode, we contour the gain and

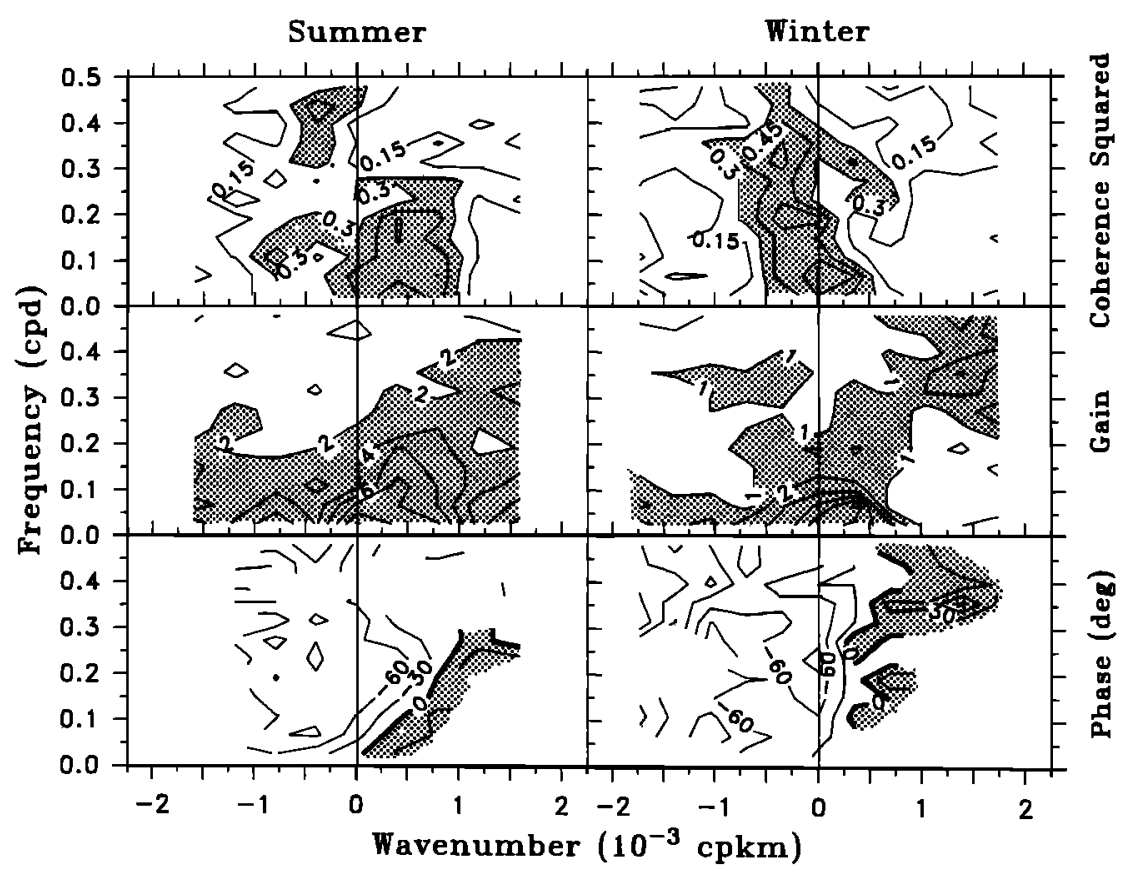

Fig. 15. Wave number-frequency coherence squared between $\tau$ and $\zeta$, and the transfer function (gain in $\mathrm{cm}$ (dyn $\left.\mathrm{cm}^{-2}\right)^{-1}$ and phase in degrees) of the $\zeta$ response to $\tau$, calculated from autospectra and cross-spectra ensemble averaged over the four summers (grid points 4 through 17) and four winters (grid points 2 through 17). The number of degrees of freedom is 40 (see Figure 7). Squared coherences of greater than 0.074 are significant to a $95 \%$ level of confidence. Phase is contoured only in that part of $(l, \omega)$ space where the coherence is statistically significant. Squared coherences greater than 0.3 , gain larger than 2 (summer) and 1 (winter), and positive phase are shaded. The $l_{m}$ and $\omega_{n}$ at which estimates are made are shown in Figure 8. 


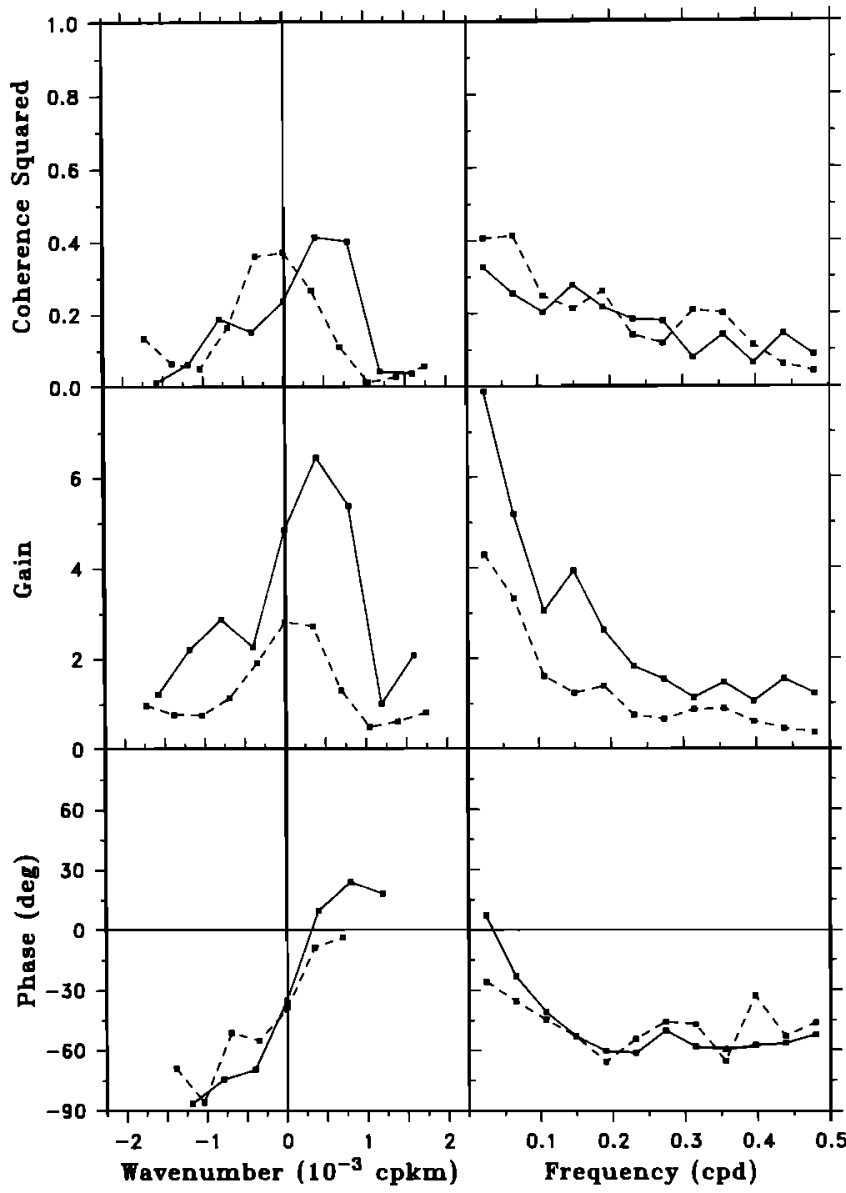

Fig. 16. (left) Integrated wave number and (right) frequency coherences squared between $\tau$ and $\zeta$ and the transfer function (gain in $\mathrm{cm}\left(\mathrm{dyn} \mathrm{cm}^{-2}\right)^{-1}$ and phase in degrees) of the $\zeta$ response to $\tau$, ensemble averaged over four summers (grid points 4 through 17; solid lines) and four winters (grid points 2 through 17; dashed lines). The wave number autospectra have 480 degrees of freedom in both seasons, and the frequency autospectra have $600(680)$ degrees of freedom for summer (winter). Wave number (frequency) coherences squared of $>0.006(>0.005)$ are statistically significant to a $95 \%$ level of confidence. Phase is graphed only where the coherence is significant.

phase of this transfer function for two values of the friction coefficient: $T_{f}=1$ and 3 days (Figure 23). (The subscripts $n=1$ are dropped for simplicity.) In these examples, we use $c=350 \mathrm{~cm} \mathrm{~s}^{-1}$, a typical value observed by Halliwell and Allen [1984] during summer 1973, and the coupling coefficient $b=\left(c T_{f}\right)^{-1}$, the latter resulting in a maximum gain of 1 (Appendix $\mathrm{C}$ ). The maximum gain and zero-phase contours both follow the linear dispersion curve, so the slope of these contours is $c=\omega / l=350 \mathrm{~cm} \mathrm{~s}^{-1}$. All other gain and phase contours are parallel to this dispersion curve. If the long-wave assumption is not made, the dispersion curve will not be straight, but the deviation from a straight line is small within that part of $(l, \omega)$ space resolved in this study [Brink et al., 1987)].

The fundamental properties of the predicted response can be elucidated by considering the response generated by a $\tau$ disturbance at a particular $|l|>0$ and $\omega>0$ (a pure sine wave that propagates alongshore). The $\zeta$ response is also a pure propagating sine wave with the same $l$ and $\omega$ as the forcing. The largest response will occur for a $\tau$ disturbance that propa- gates alongshore at speed $\omega / l=c_{\tau}=c$, and this response will be exactly in phase with the $\tau$ disturbance. If $c_{\tau}$ differs from $c$, the phase, which can be characterized as either a space or time lag, will be nonzero. Positive (negative) phase indicates that the response wave is displaced alongshore from the $\tau$ wave in the direction toward (opposite from) which the $\tau$ wave is propagating, resulting in the $\zeta$ response leading (lagging) $\tau$ in time at a given $y$. Positive phase is observed for $0 \leq c_{\tau}<c$, representing poleward propagation of the $\tau$ wave at a speed slower than the free-wave phase speed and corresponding to the shaded regions of the phase plots in Figure 23. Negative phase is observed for $c_{\tau}>c$, representing poleward propagation of the $\tau$ wave at a speed faster than the free-wave phase speed and corresponding to the unshaded regions of the positive $l$ side of the phase plots, and also for $c_{\tau}<0$, representing equatorward propagation of the $\tau$ wave and corresponding to the negative $l$ side of the phase plots. The response wave always propagates along the coast at the same speed as the $\tau$ wave, but the spatial (and resulting temporal) lag of the response depends on the difference between $c_{\tau}$ and $c$, with the lag equaling zero if $c_{\tau}=c$.

Increasing $T_{f}$ has several effects. The maximum gain increases, since it equals $c T_{f} b$ (Appendix $C$ ). The response far from the dispersion curve in $(l, \omega)$ space becomes relatively smaller and has a larger phase lead or lag. In the limit as $T_{f} \rightarrow \infty$, the gain approaches infinity along the dispersion curve and zero elsewhere in $(l, \omega)$ space, and the phase approaches $\pm 90^{\circ}$ everywhere in $(l, \omega)$ space except on the dispersion curve. Increasing $c$ will increase the slopes of the contours in Figure 23 and will also increase the maximum gain, since it is a linear function of $c$.

There are qualitative similarities between the observed gain and phase (Figure 15) and the gain and phase predicted by (1) for one mode. Both observed and predicted gains are largest along a ridge that follows a line of constant $\omega / l$ for positive $l$, and both observed and predicted phases change sign on opposite sides of this ridge, although the observed winter sign change is poorly resolved owing to low coherence in that part of $(l, \omega)$ space. The prediction that a near-resonant response can occur due to $\tau$ disturbances that propagate poleward at speed $c=\omega / l$ is confirmed by the data. The good coherence between $\tau$ and $\zeta$ at $|l| \leq 1.1 \times 10^{-3} \mathrm{cpkm}$, coupled with the qualitative similarities between observed and predicted response properties, indicates that large-scale wind forcing of $\zeta$ is very important along the west coast of North America in both summer and winter, and that the response to this forcing has many properties that are predicted for forced large-scale coastally trapped waves.

At sufficiently small $\omega$, coastally trapped wave energy can be propagated offshore by planetary Rossby waves as is shown in a barotropic model by Dorr and Grimshaw [1986], so (1) becomes invalid as $\omega \rightarrow 0$. However, theoretical models that accurately describe the nature of this behavior for general stratified situations are not available. Thus it is not clear at precisely what frequencies or in what form this breakdown of (1) will occur. This process may influence the observed response properties in $(l, \omega)$ space near the smallest $\omega$ resolved (Figure 15). In particular, the decay rate of the forced wave response may be larger at these small $\omega$, tending to decrease the amplitude of the response. The observed maximum in gain near the dispersion curve increases with decreasing $\omega$ down to the smallest $\omega$ resolved $(0.025 \mathrm{cpd})$ in summer, and to the 


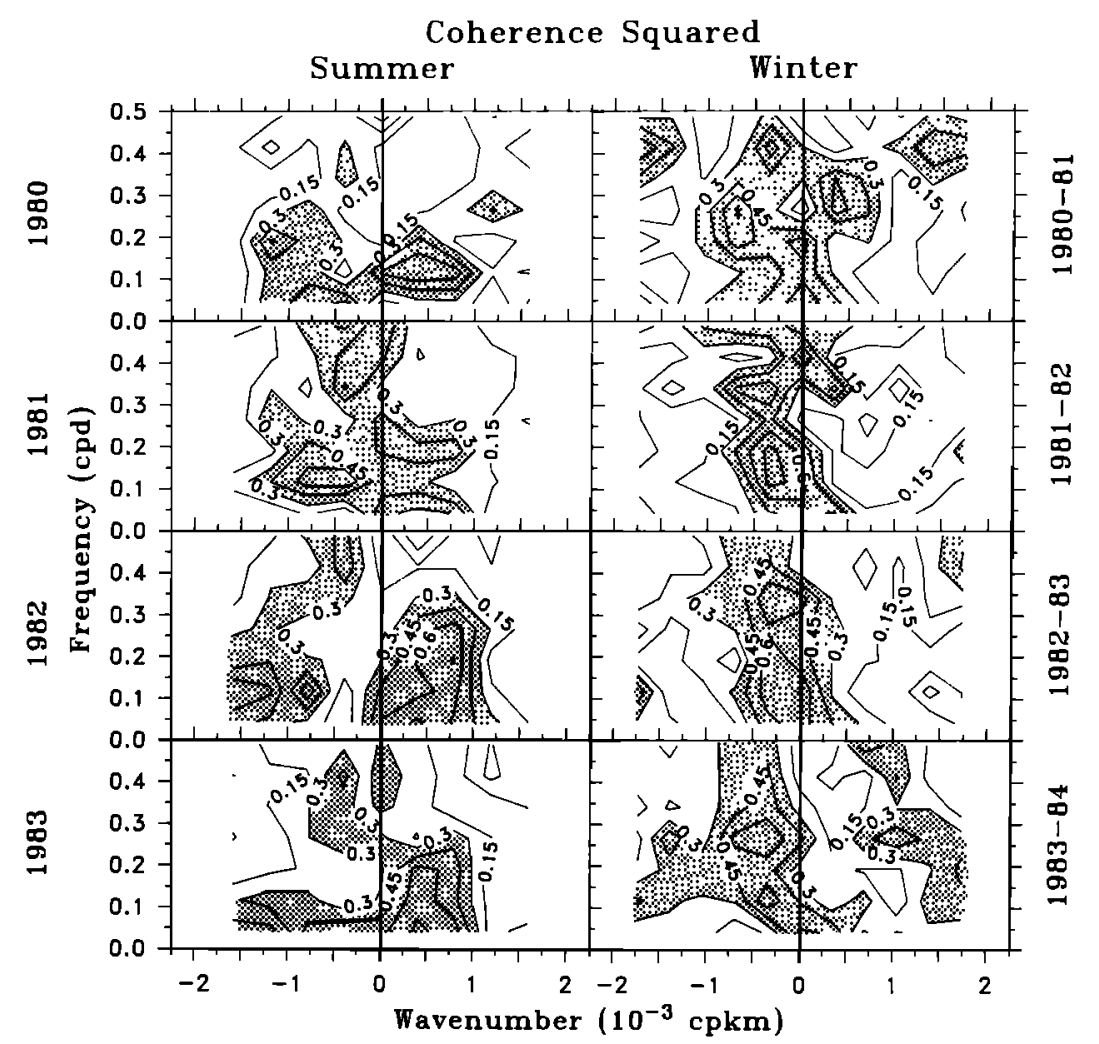

Fig. 17. Wave number-frequency coherence squared between $\tau$ and $\zeta$ for the individual four summers (grid points 4 through 17) and four winters (grid points 2 through 17). The number of degrees of freedom is 18 (see Figure 9). Squared coherences of greater than 0.16 are statistically significant to a $95 \%$ level of confidence, and those of greater than 0.3 are shaded. The $l_{m}\left(\omega_{n}\right)$ at which estimates are made are shown in Figure 8 (Figure 13).

second-smallest $\omega$ resolved $(0.066 \mathrm{cpd})$ in winter (Figure 15). In addition, the observed maximum winter gain decreases only slightly from $\omega=0.066 \mathrm{cpd}$ to $\omega=0.025 \mathrm{cpd}$. Consequently, offshore propagation of energy due to Rossby waves apparently does not have a clearly identifiable effect on the observed response.

We attempt to estimate free-wave parameters in (1) by comparing the observed transfer functions (Figure 15) to the theoretical transfer functions, but as we show in Appendix D, the presence of noise in the data limits our ability to do this. In particular, the observed gain functions are reduced in magnitude by the presence of noise in the $\tau$ data set only. Therefore observed and theoretical gain functions should be compared only in those parts of $(l, \omega)$ space where calculated $\tau$ is reasonably accurate, i.e., where calculated and measured $\tau$ are highly coherent with each other (Figure 14). This noise bias may be a major reason why the region of relatively large gain along the dispersion line is confined to smaller $l$ and $\omega$ in winter, since the winter coherence between measured and calculated $\tau$ decreases rapidly with increasing $l$ and $\omega$ along this line (Figure 14). In contrast, noise in the $\tau$ and $\zeta$ data sets has no effect on the observed phase functions (Appendix D), so direct comparisons of observed and theoretical phase functions will probably be more useful.

Because of this noise effect, we do not try to obtain estimates of the parameters of (1) by statistically fitting the predicted to observed transfer functions. Instead, we make rough estimates by visual comparison of the phase functions in Figures 15 and 23 . In summer, $\hat{c}$ appears to be roughly $300 \mathrm{~cm}$ $\mathrm{s}^{-1}$, based on the slope of the zero-phase contour, and $\hat{T}_{f}$ appears to fall between 1 and 3 days, based on the phase gradient in the vicinity of the dispersion line. Estimates are more difficult to make for winter since the zero-phase contours are not as well defined as in summer, but $\hat{c}$ appears larger and $\hat{T}_{f}$ appears roughly the same in winter as opposed to summer. These $\hat{c}$ estimates agree with those made from the space-time correlation functions (section 3.2, Figure 4). We show later that the phase gradients can be substantially altered if more than one mode is present, so we cannot consider these estimates of $\hat{T}_{f}$ to be accurate. We do not estimate $b$ because we lack confidence in how well calculated $\tau$ represents $\tau$ variance along the coast.

Since the gain is proportional to $c b T_{f}$, and since $\hat{c}$ does not appear to be smaller in winter, the observed winter decrease in gain must be caused by decreases in $b$ or $T_{f}$. The magnitude of the coupling coeflicient $b$ depends on the basic stratification [Brink, 1982], and this stratification changes substantially between summer and winter. According to the analysis of Grant and Madsen [1979], the friction time scale $T_{f}$ will probably be smaller in winter because of larger bottom stress due to increased storminess in the northeast Pacific and the resulting increase in surface gravity wave energy over the continental shelf. The fact that $\tau$ variance increases much more in winter than in summer poleward of the CODE site $(y>0)$ than does $\zeta$ variance (Figure 3 ) indicates that the winter decrease in gain may be $y$-dependent. Since winter storminess increases toward the north, larger bottom stress from increased surface gravity wave energy may produce a $y$-dependent winter decrease in $\hat{T}_{f}$ 


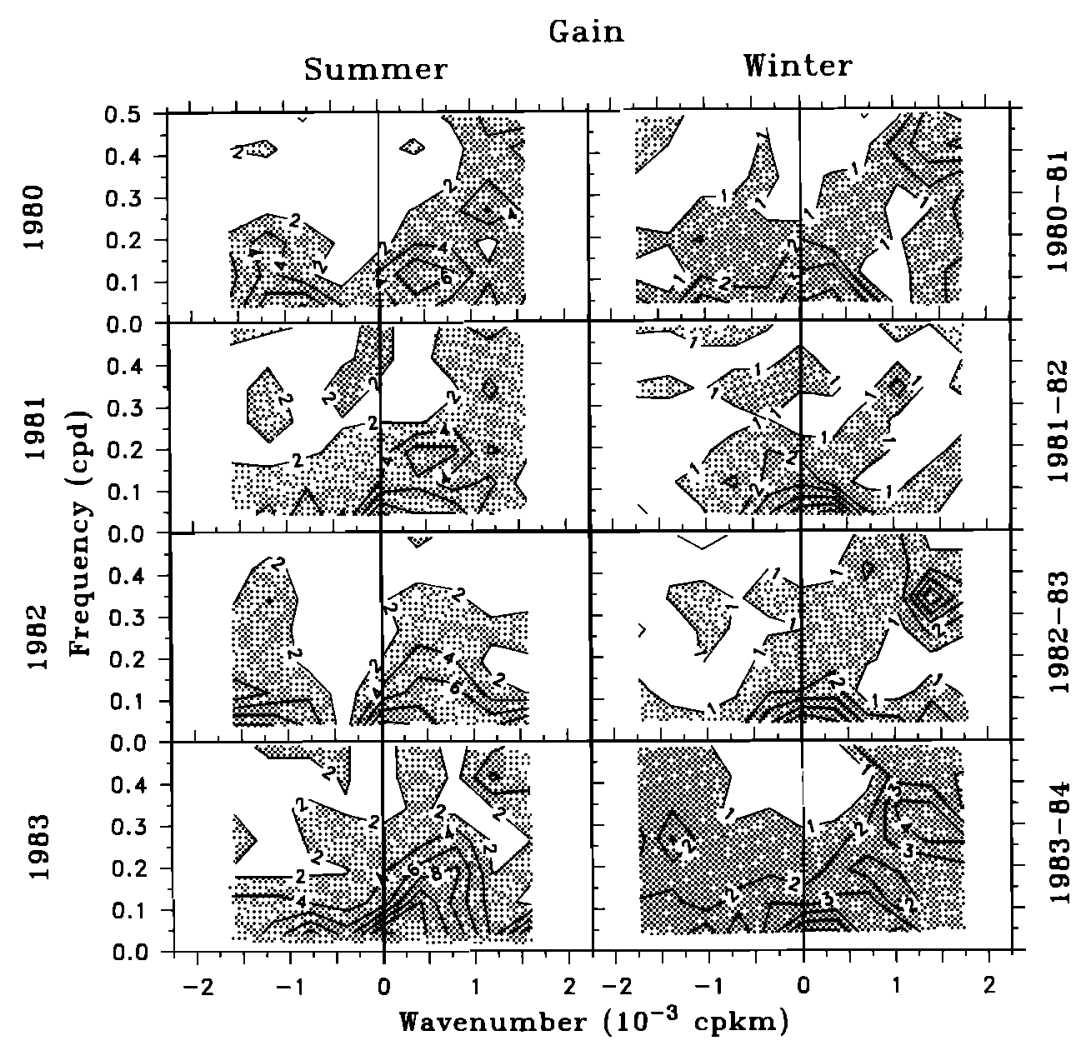

Fig. 18. Wave number-frequency gain in $\mathrm{cm}\left(\mathrm{dyn} \mathrm{cm}^{-2}\right)^{-1}$ of the transfer function of the $\zeta$ response to $\tau$ for the individual four summers (grid points 4 through 17) and four winters (grid points 2 through 17). The number of degrees of freedom is 18 (see Figure 9). Gain of greater than 2 (summer) and 1 (winter) is shaded. The $l_{m}\left(\omega_{n}\right)$ at which estimates are made are shown in Figure 8 (Figure 13).

that could at least partly explain this $y$-dependence of the seasonal change in gain.

One major difference between the observed and predicted response properties does exist: The maximum predicted gain is constant along the dispersion curve $\omega / l=c$, while the observed gain decreases substantially along this line as $l$ and $\omega$ increase. This decrease is observed in parts of $(l, \omega)$ space where coherence is large, so we believe that this phenomenon is real and does not depend on the noise effect. In summer, for example, $\hat{\gamma}^{2}=0.46$ and $\hat{G}=12.2 \pm 3.8 \mathrm{~cm}\left(\mathrm{dyn} \mathrm{cm}^{-2}\right)^{-1}$ at $l=0, \omega=0.025 \mathrm{cpd}$, while $\hat{\gamma}^{2}=0.49$ and $\hat{G}=5.0 \pm 2.3 \mathrm{~cm}$ (dyn $\left.\mathrm{cm}^{-2}\right)^{-1}$ at $l=7.9 \times 10^{-4} \mathrm{cpkm}, \omega=0.19 \mathrm{cpd}$, where the $95 \%$ error bars for $\hat{G}$ are indicated. The fact that the coherence is not significantly different indicates that the fraction of noise in the $\tau$ and $\zeta$ data sets does not change much (Appendix C), yet the gain decreases by an amount that is large compared to the width of the error bars. We conclude that in summer, gain decreases significantly along the dispersion line with increasing $l$ and $\omega$.

In winter, coherence decreases rapidly along the dispersion line, so we cannot make the same conclusion. However, coherence remains large for $l=0$ while gain decreases with increasing $\omega$. For example, $\hat{\gamma}^{2}=0.60$ and $\hat{G}=5.7 \pm 2.1 \mathrm{~cm}$ (dyn $\left.\mathrm{cm}^{-2}\right)^{-1}$ at $l=0, \omega=0.025 \mathrm{cpd} \hat{\gamma}^{2}=0.65$ and $\hat{G}=2.1 \pm 0.5$ $\mathrm{cm}\left(\mathrm{dyn} \mathrm{cm}^{-2}\right)^{-1}$ at $l=0, \omega=0.19 \mathrm{cpd}$. We therefore establish that gain decreases with increasing $\omega$ in winter, but cannot establish whether gain decreases with both $l$ and $\omega$.
Possible mechanisms that may cause this observed decrease in gain include the $y$-dependence of parameters in (1), an $(l$, $\omega)$-dependent friction that may exist if the linear friction term in (1) is invalid, and a significant contribution to the response by higher modes. The effects of $y$-dependent parameters were explored by numerically integrating (1) for different $c(y), T_{f}(y)$, and $b(y)$ with $\tau$ from winter $1981-1982$ and summer 1982 as the forcing functions, using the method described in Halliwell and Allen [1984] extended to handle $y$-dependent parameters, and then computing the transfer functions for the numerical solutions. Variable parameters distort the gain and phase by only a small amount (not shown), and this mechanism appears incapable of accounting for the large observed decrease in maximum gain. This result is in agreement with Chapman [1987], who found that in integrations of (1), predictions of a response variable at a given $y$ show little difference in quality whether $y$-dependent or averaged parameters are used.

The responses predicted by two recent models illustrate that the effect of friction can depend on $l$ and $\omega$ if the assumptions required to derive the linear friction term in (1) are relaxed. The two-layer Kelvin wave model of Allen [1984] predicts an increasing gain with decreasing $l$ and $\omega$ that is qualitatively consistent with observations, but most of the increase is confined to much smaller $|l|$ and $\omega$ than is observed. However, the stochastic model of the wind-driven response of a continuously stratified continental shelf and slope presented by Brink et al. [1987] predicts a decreasing resonant response with de- 


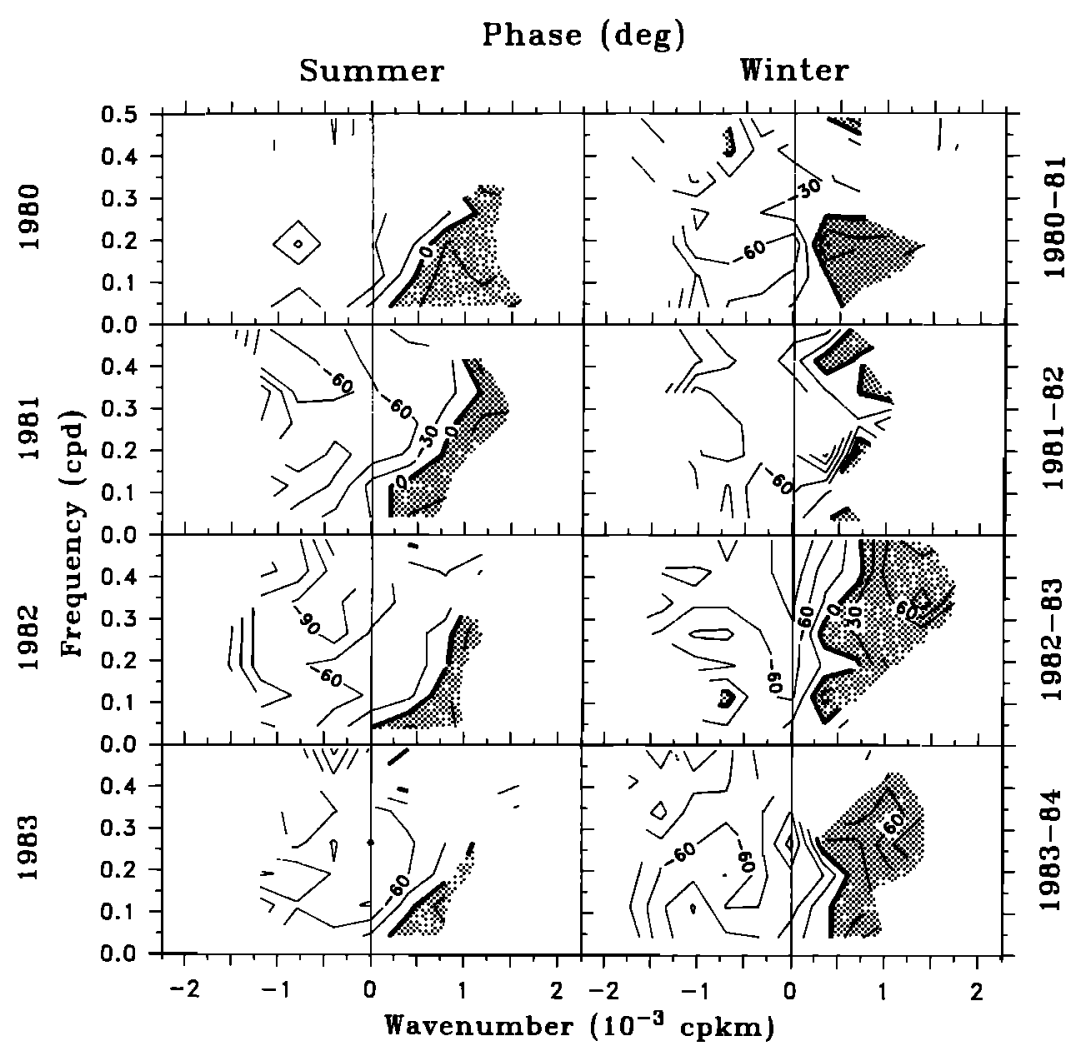

Fig. 19. Wave number-frequency phase in degrees of the transfer function of the $\zeta$ response to $\tau$ for the individual four summers (grid points 4 through 17) and four winters (grid points 2 through 17). The number of degrees of freedom is 18 (see Figure 9). Phase is contoured only in that part of $(l, \omega)$ space where the coherence is statistically significant. Phase greater than $0(\zeta$ leads $\tau)$ is shaded. The $l_{m}\left(\omega_{n}\right)$ at which estimates are made are shown in Figure 8 (Figure 13).

creasing $\omega$, not the increasing resonant response that is observed. The $(l, \omega)$ dependence of friction can therefore be strongly dependent on the model assumptions and must be further studied.

\subsection{Response Predicted by (1) for More Than One Mode}

We consider the properties of the total $\zeta$ response in $(l, \omega)$ space that result if more than one coastally trapped wave contributes significantly to this response. Parameters of (1) for $\zeta$ as the response variable, calculated using the model of Brink [1982] for the CODE site (K. H. Brink, personal communication, 1986) and also averaged along the west coast of the United States between San Diego $(y=-938 \mathrm{~km})$ and the CODE site $(y=0)$ [Chapman, 1987], are listed in Table 7 along with the maximum gain for each mode. In calculating these parameters, Brink and Chapman used a bottom friction coefficient $r=0.05 \mathrm{~cm} \mathrm{~s}^{-1}$ at all locations along the coast. For the CODE site, the maximum gain of modes 2 through 4 are $33 \%, 11 \%$, and $6 \%$ of the maximum gain of mode 1 . For the west coast, the corresponding percentages for modes 2 and 3 are $32 \%$ and $17 \%$. These estimates indicate that modes 2 and 3 may make a substantial contribution to the total observed $\zeta$ response in the CODE large-scale domain.

The predicted response properties for multiple uncoupled modes can be readily studied since the $(l, \omega)$ transfer function of the total response is simply the sum of the individual transfer functions. These total transfer functions are calculated for the CODE site and the west coast using the parameters for all modes listed in Table 7 , and the gain and phase of these functions are contoured in Figure 24. Individual ridges of large gain are evident at large positive $l$, each following a line given by $\omega / l=c_{n}$. At very small $\omega$ and $l$, the ridges merge into one broader ridge and become indistinct from each other. Sharp phase changes are observed near the dispersion curves.

The ridges of large gain are substantially narrower, and the phase changes are sharper, than are observed for the single mode (Figure 23) because the $T_{f n}$ values in Table 7 are much larger than the $T_{f}$ values used for the single-mode cases. These theoretically estimated $T_{f}$ values are also much larger than both the estimate of 1 to 3 days made using Figure 15 and the estimate of 4.25 days made by Halliwell and Allen [1984] for summer 1973. To illustrate the effects of changing $T_{f n}$ on the multiple-mode response, we calculate gain and phase using the parameters in Table 7, but with $T_{f 1}$ set to 2.5 days (reduced to $45 \%$ of its estimated theoretical value for the CODE site and $26 \%$ of its estimated theoretical value for the west coast) and with the ratios $T_{f n} / T_{f 1}$ preserved (Figure 25). With $T_{f}$ reduced, the individual ridges of large gain are substantially broader and therefore more nearly blend together to appear as one broad ridge. The single ridge dominates for $l \leq 1.1 \times 10^{-3}$ cpkm, and the only apparent contribution from higher modes is to cause the gain to decrease with increasing $\omega$ and $l$, as observed in the data. Contributions to the $\zeta$ response from higher modes may therefore contribute to the observed decrease in gain with increasing $\omega$ and $l$. The phase functions in 


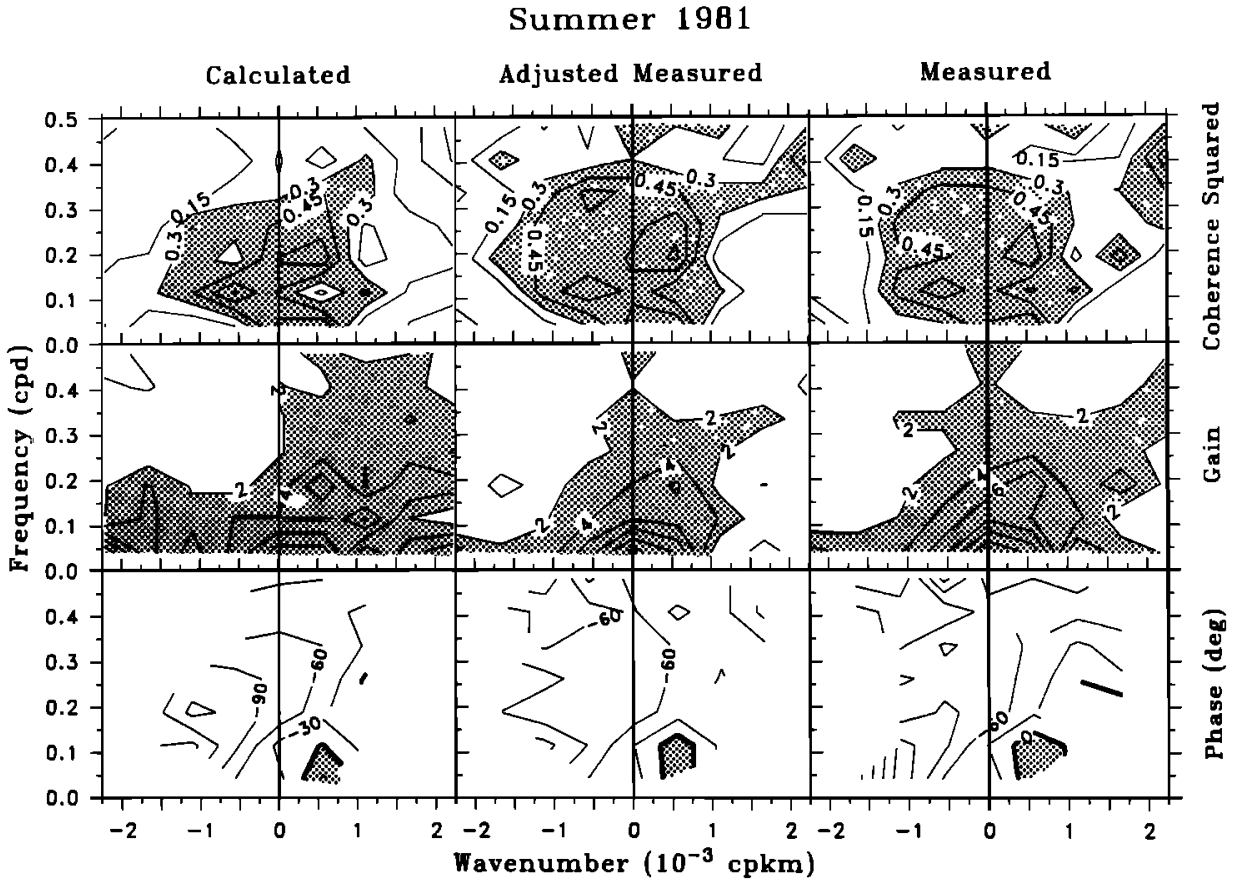

F1g. 20. Wave number-frequency coherence squared between $\tau$ and $\zeta$, and transfer functions (gain in $\mathrm{cm}\left(\mathrm{dyn} \mathrm{cm}^{-2}\right)^{-1}$ and phase in degrees) of the $\zeta$ response to $\tau$, at grid points 4 through 13 for calculated, adjusted measured, and measured $\tau$ during summer 1981. The number of degrees of freedom is 18 (see Figure 12). Squared coherences of greater than 0.16 are significant to the $95 \%$ level of confidence. Phase is contoured only in that part of $(l, \omega)$ space where the coherence is statistically significant. Squared coherences greater than 0.3 , gain larger than 2 , and positive phase $(\zeta$ leads $\tau)$ are shaded. The $l_{m}$ and $\omega_{n}$ at which estimates are made are shown in Figure 13.

Figure 25 also better resemble the observed phase functions in Figure 15 than do those in Figure 24.

With more than one mode present, phase contours can be substantially distorted from those predicted for a single mode, so it is hazardous to estimate $T_{f}$ for the dominant first mode from an observed phase function if higher modes are signifi- cant. Also, the $(l, \omega)$ resolution in the data analyses is too coarse to resolve the narrow ridges of large gain and the very large phase gradients predicted when large $T_{f n}$ are assumed. Because of these considerations, the crude estimate of 1 to 3 days that we made earlier cannot be used to invalidate the larger values determined by theory. In contrast, higher modes

Winter 1981-82

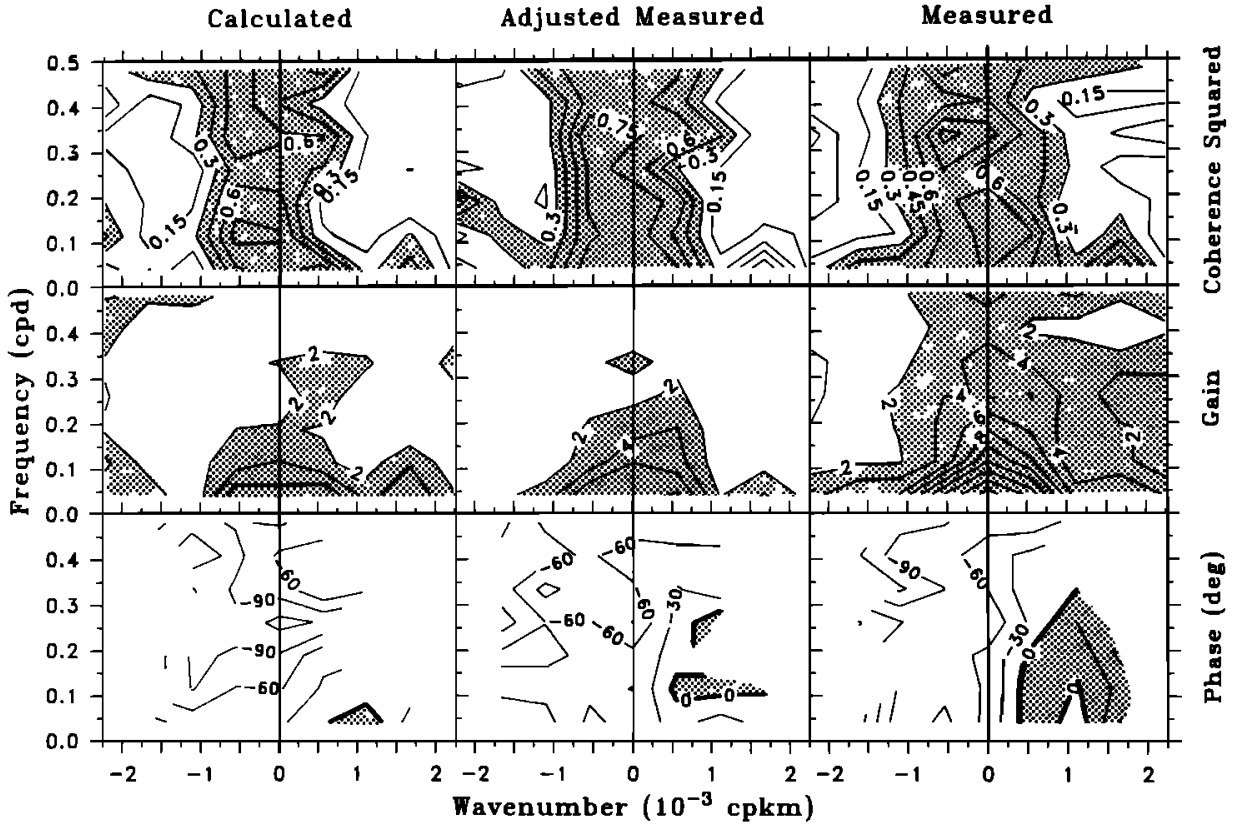

Fig. 21. Same as Figure 20, but for winter 1981-1982. 


\section{Summer 1982}

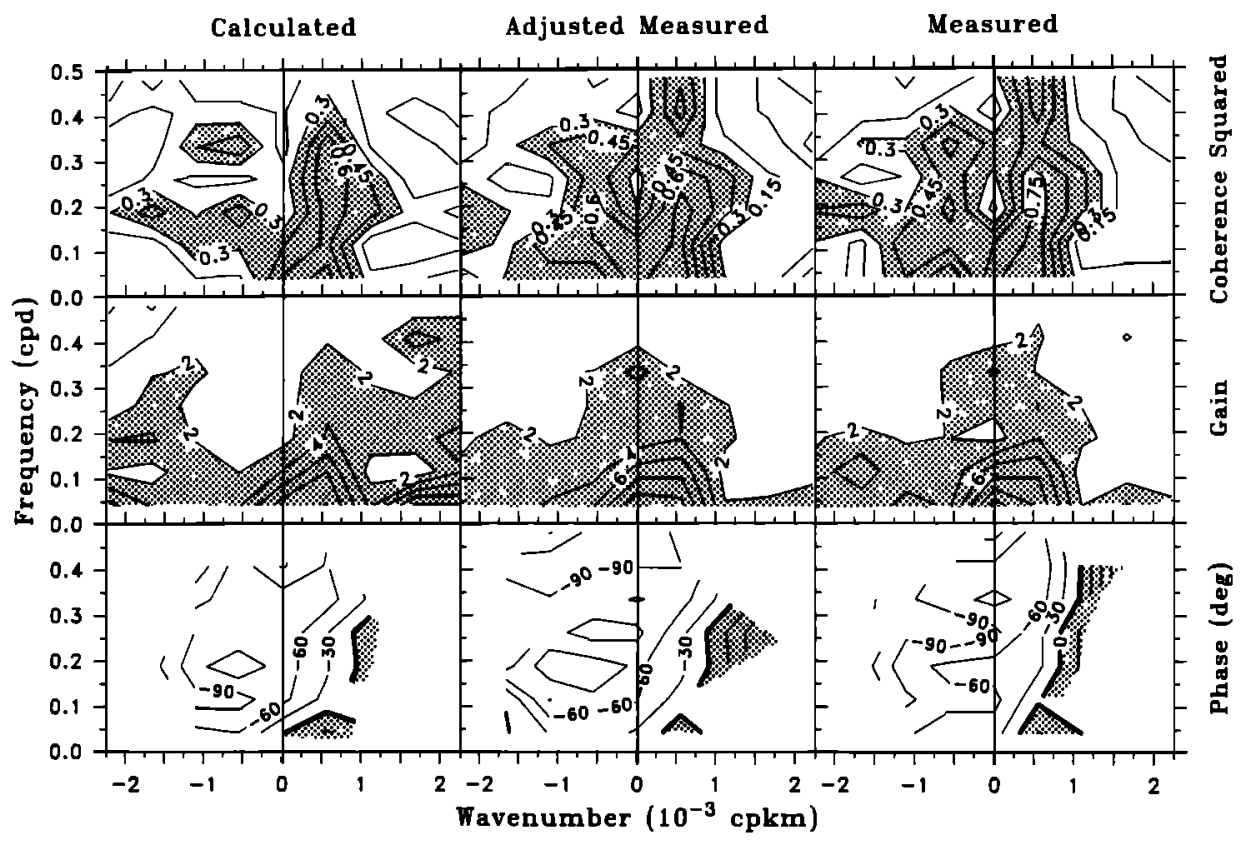

Fig. 22. Same as Figure 20, but for summer 1982.

appear to have little effect on the slope of the zero-phase contour that follows the first-mode dispersion line, so phase speed estimates made from Figure 15 may be reasonably accurate estimates of an effective first-mode phase speed.

We also tested if frictional coupling among the modes [Brink, 1982; Chapman, 1987] might also act to smooth out the gain and phase functions in Figure 24 near the dispersion lines and perhaps contribute to the observed decrease of gain with increasing $l$ and $\omega$. The influence of frictional coupling on the theoretical transfer function is discussed in Appendix C, along with the method we used to test this effect. We calculated transfer functions using $\beta=0.3$ and $\beta=0.6$ in (C12) to generate mode coupling coefficients with magnitudes typical of those observed by Chapman [1987]. The results (not shown) indicate that mode coupling does not smooth the gain and phase functions significantly, nor does it cause the gain to decrease with increasing $l$ and $\omega$. We can therefore conclude that although frictional coupling coefficients may have significant magnitude, this coupling has a small influence on the predicted properties of the response in $(l, \omega)$ space. This is consistent with the results of Chapman [1987], who noted that the inclusion of mode coupling effects in integrations of firstorder wave equations did not significantly change the predicted coastal circulation response.

$$
\begin{gathered}
c=350 \mathrm{~cm} \mathrm{~s}^{-1} \\
\left(\mathrm{cT}_{\mathrm{f}}\right) \mathrm{b}=1 \mathrm{~cm}\left(\mathrm{dy} \mathrm{cm}^{-2}\right)^{-1}
\end{gathered}
$$

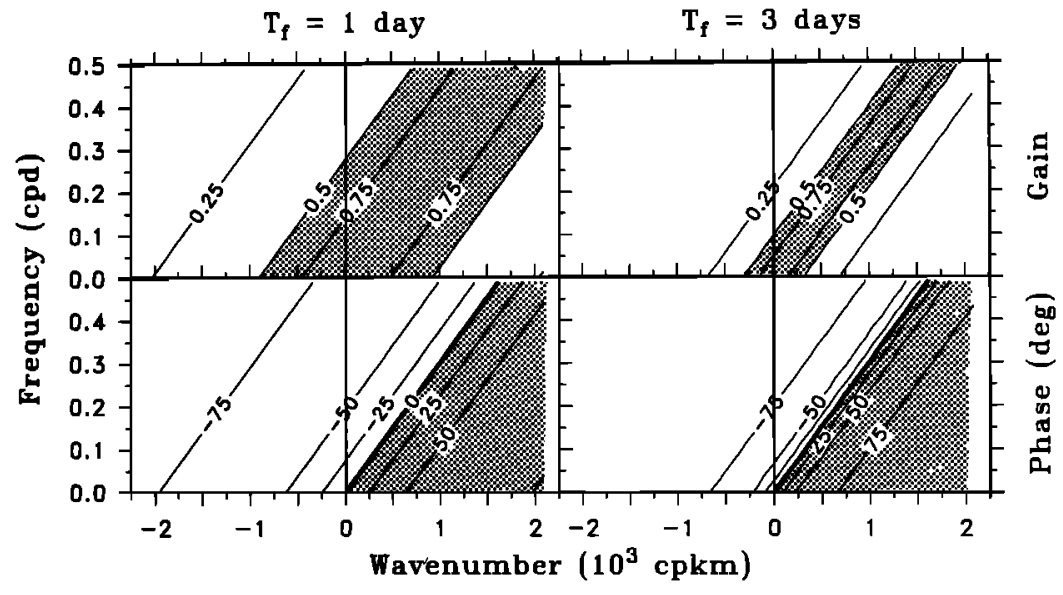

Fig. 23. Transfer lunctions (gain and phase) of the $\zeta$ response to $\tau$ in wave number-frequency space predicted by (1) for one coastally trapped wave mode calculated for two values of $T_{f}(1$ and 3 days). The coupling coeflicient $b$ is chosen such that the maximum gain is $1 \mathrm{~cm}\left(\mathrm{dyn} \mathrm{cm}^{2}\right)^{-1}$. Values of gain $>0.5$ and positive phase are shaded. 
TABLE 7. Parameters of (1) Calculated by K. H. Brink (personal communication, 1986) for the CODE Site and D. C. Chapman and

K. H. Brink (personal communication, 1986) for the West Coast Between San Diego and the CODE Site Using the Model of Brink [1982]

\begin{tabular}{|c|c|c|c|c|c|}
\hline \multirow[b]{2}{*}{ Location } & \multirow[b]{2}{*}{ Parameter } & \multicolumn{4}{|c|}{ Mode } \\
\hline & & 1 & 2 & 3 & 4 \\
\hline \multirow[t]{4}{*}{ CODE site } & $c_{n}, \mathrm{~cm} \mathrm{~s}^{-1}$ & 299 & 153 & 84 & 55 \\
\hline & $10^{8} b_{n},\left(\text { dyn }_{\mathrm{cm}^{-2}}\right)^{-1}$ & 5.85 & 3.41 & 1.34 & 0.92 \\
\hline & $T_{S n}$, days & 5.52 & 6.00 & 9.35 & 11.0 \\
\hline & $\underset{\left.\mathrm{cm}^{-2}\right)^{-1}}{\operatorname{maximum}}$ gain $\mathrm{cm}(\mathrm{dyn}$ & 8.34 & 2.70 & 0.91 & 0.48 \\
\hline \multirow[t]{4}{*}{ West coast } & $\mathcal{c}_{n}, \mathrm{~cm} \mathrm{~s}^{-1}$ & 350 & 176 & 111 & \\
\hline & $10^{8} b_{n},\left(\text { dyn } \mathrm{cm}^{-2}\right)^{-1}$ & 4.17 & 4.17 & 3.09 & \\
\hline & $T_{f r}$, days & 9.45 & 6.26 & 6.82 & \\
\hline & $\underset{\left.\mathrm{cm}^{-2}\right)^{-1}}{\operatorname{maximum}}$ gain, $\mathrm{cm}(\mathrm{dyn}$ & 11.9 & 3.97 & 2.02 & \\
\hline
\end{tabular}

The values for the west coast are averaged over 17 subdomains along the west coast of North America as was described by Chapman [1987].

\subsection{One-Dimensional Response Properties Predicted by (1)}

Using the observed winter and summer $(l, \omega)$ autospectra of $\tau$ (Figure 7), we calculate the one-dimensional coherence squared and gain as functions of $l$ and $\omega$ predicted by (1) as described in Appendix $\mathrm{C}$, assuming that one mode dominates the response. We calculate these functions using $c=350 \mathrm{~cm}$ $\mathbf{s}^{-1}$, and $T_{f}=1$ day (Figure 26) and $T_{f}=3$ days (Figure 27), the parameters used to predict $(l, \omega)$ transfer functions (Figure 23). The coherence predicted by (1), which is a constant equal to one in $(l, \omega)$ space, is generally less than 1 when calculated as a function of $l$ or $\omega$ (Appendix $C$ ). A similar reduction of coherence appears in the observed one-dimensional coherence functions (Figure 16). For example, summer $\hat{\gamma}^{2}\left(l_{m}, \omega_{n}\right)$ in Figure 15 is greater than 0.45 at $\omega=0.025 \mathrm{cpd}$ for $-1.5 \times 10^{-3}<l<1.5 \times 10^{-3} \mathrm{cpkm}$, which contains most of the $\tau$ and $\zeta$ variance at that $\omega$, yet summer $\hat{\gamma}^{2}(\omega)$ in Figure 16 is only 0.29 at $\omega=0.025 \mathrm{cpd}$. The predicted one-dimensional coherence (Figure 26) is approximately constant as a function of $\omega$, but it varies as a function of $l$, with the smallest coher-

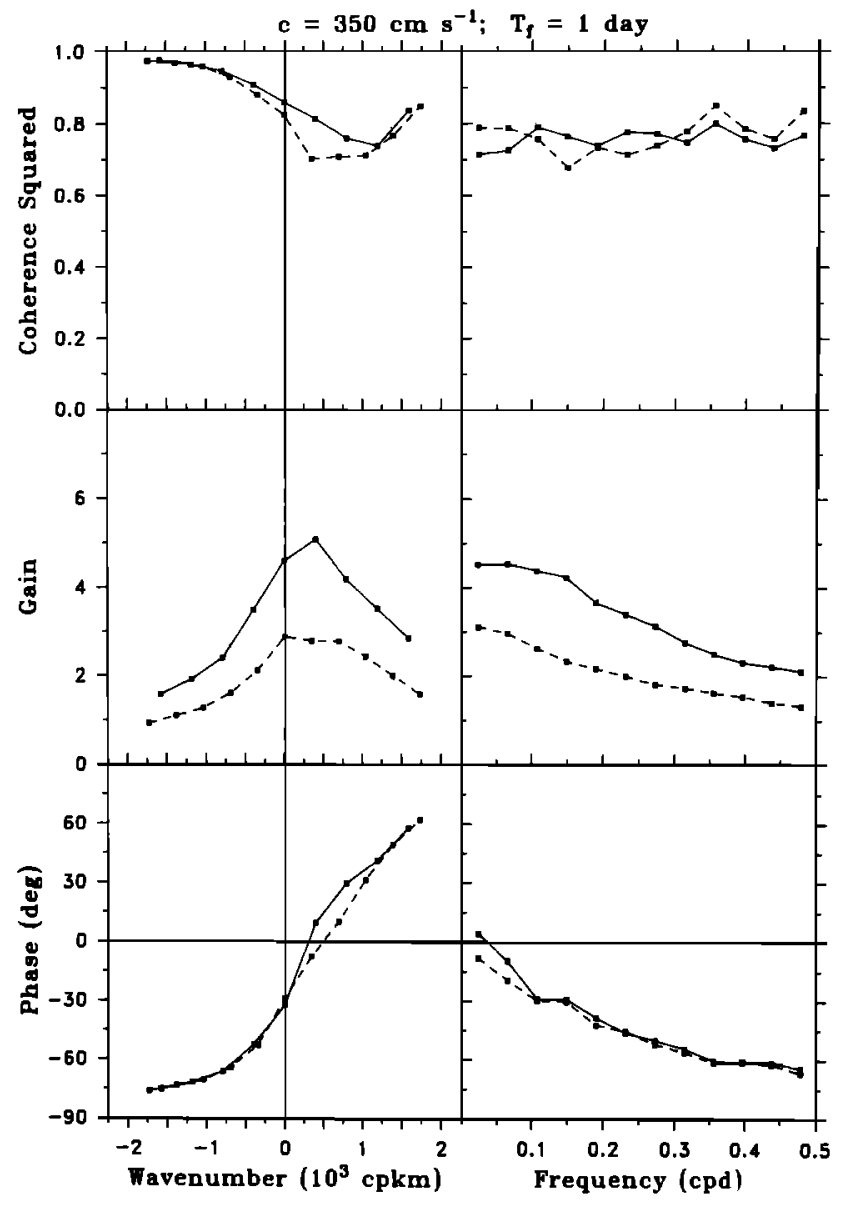

Fig. 25. Same as Figure 24, except that the $T_{f n}$ from Table 7 have been reduced in magnitude as shown. The maximum gain for each mode is the same as shown in Figure 24. Positive phase is shaded.

ence found for $0 \leq l<1.5 \times 10^{-3} \mathrm{cpkm}$. Both the $l$ and $\omega$ coherence functions decrease with increasing $T_{f}$, with an especially large decrease observed in the $l$ coherence function for $0 \leq l<1.5 \times 10^{-3} \mathrm{cpkm}$. Increasing $T_{f}$ increases the relative importance of distantly forced $\zeta$ fluctuations, thus decreasing the coherence between $\zeta$ and $\tau$, at all locations along the coast. The predicted and observed one-dimensional coherence functions differ substantially, presumably because noise in the $\tau$

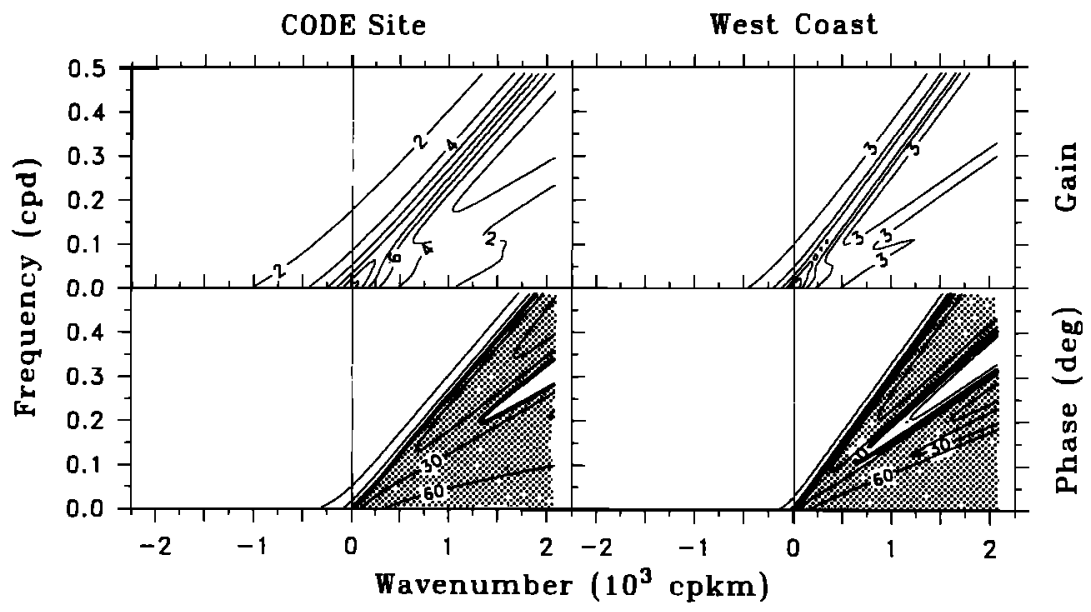

Fig. 24. Transfer functions (gain and phase) of the $\zeta$ response to $\tau$ in wave number-frequency space predicted by (1) for several coastally trapped wave modes calculated using the parameters in Table 7 . Positive phase is shaded. 


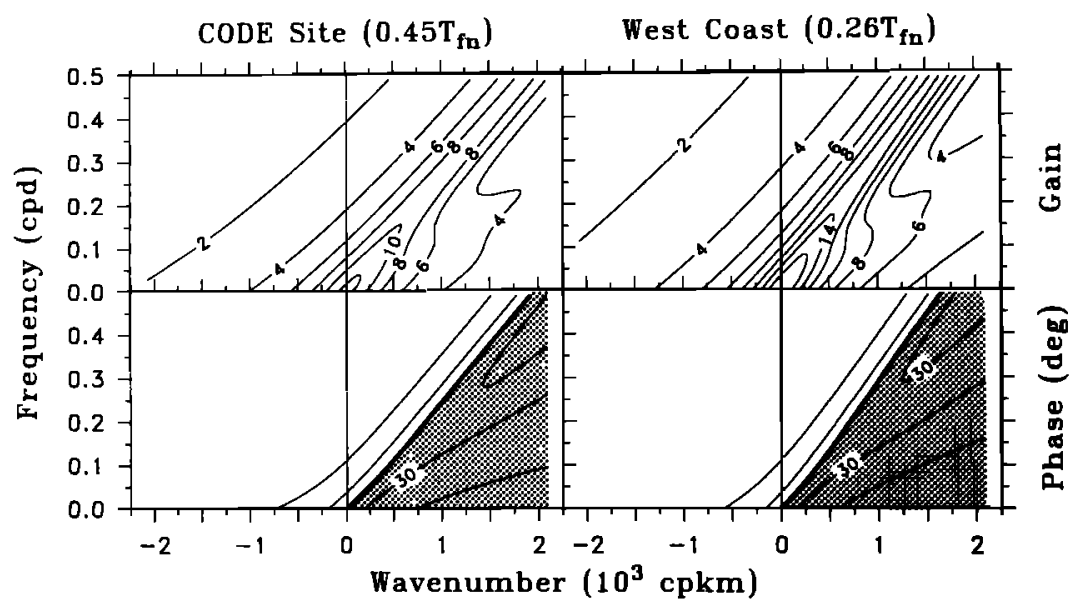

F1g. 26. (left) Integrated wave number and (right) frequency coherence squared between $\tau$ and $\zeta$, and the transfer function (gain in $\mathrm{cm}$ (dyn $\left.\mathrm{cm}^{-2}\right)^{-1}$ and phase in degrees) of the $\zeta$ response to $\tau$, calculated from (1) as is described in Appendix $C$ using the summer (solid lines) and winter (dashed lines) autospectra of $\tau$ presented in Figure 7 . For this calculation, we use $c=350 \mathrm{~cm} \mathrm{~s}{ }^{-1}$ and $T_{f}=1$ day to calculate the $(l, \omega)$ transfer function from (1), and we choose $b$ so that the $l$ gain functions in both seasons at $l=0$ have the same magnitudes as those for the corresponding observed gain functions in Figure 16.

and $\zeta$ data sets substantially reduces the observed coherence (Appendix D), particularly for $|l|>1.1 \times 10^{-3} \mathrm{cpkm}$.

The predicted $l$ gain functions (Figures 26 and 27 ) tend to be relatively large for positive $l$, and the $\omega$ gain functions decrease with increasing $\omega$. In general, there is a better resem-

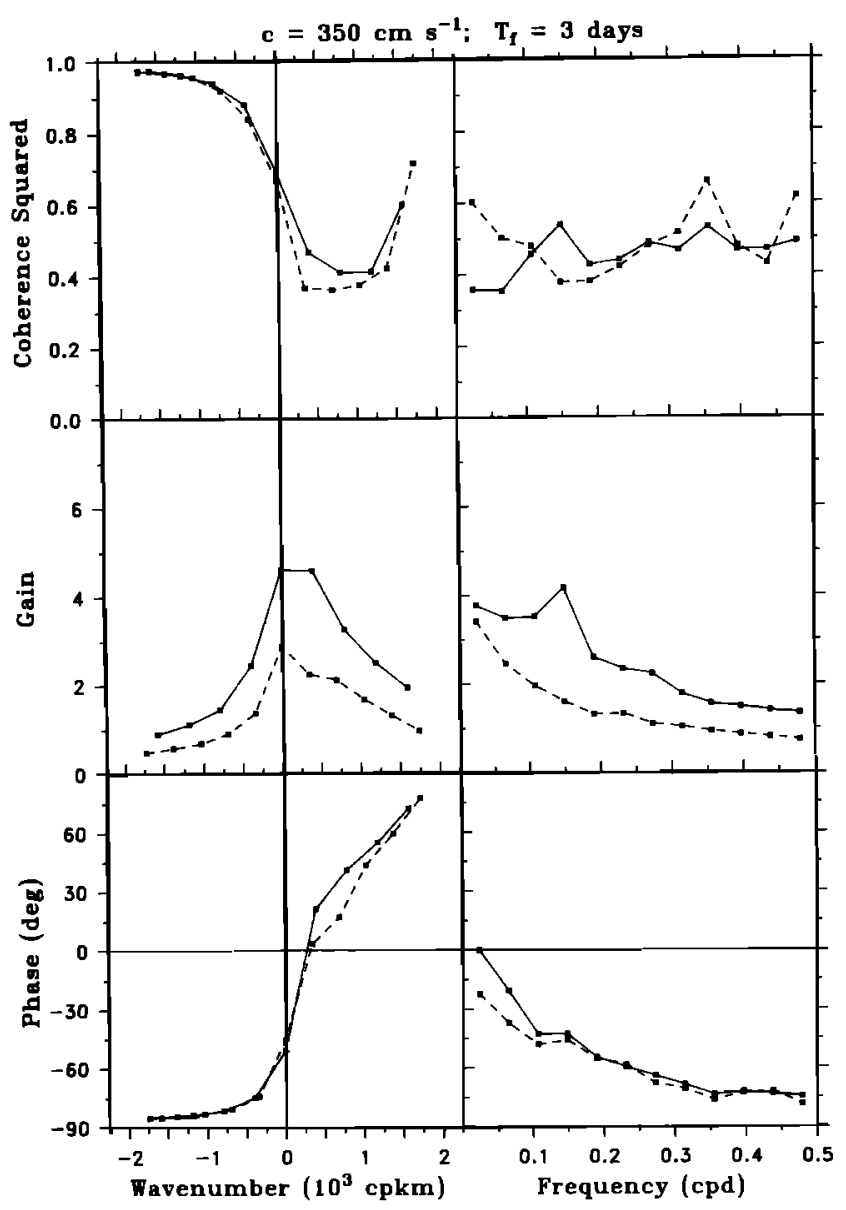

Fig. 27. Same as Figure 26, except that $T_{f}=3$ days. blance between observed and predicted gain functions than between the corresponding coherence functions. However, the corresponding observed gain functions (Figure 16) are larger than the predicted gain functions for the smallest positive $l$ resolved and for small $\omega$. This is consistent with the inability of (1) to predict the increase in maximum gain in $(l, \omega)$ space for decreasing $l$ and $\omega$ observed in Figure 15. Increasing $T_{f}$ does not change the shape of the $l$ gain functions very much. Increasing $T_{f}$ from 1 to 3 days introduces a relative maximum to the predicted summer $\omega$ gain function for $\omega=0.15 \mathrm{cpd}$ (Figure 27), where a relative maximum is found in the observed summer gain (Figure 16). Increasing $T_{f}$ also increases the magnitude of the slope of the predicted winter $\omega$ gain function for small $\omega$, in better agreement with the observed winter gain. The predicted phase functions are qualitatively very similar to the observed phase functions, changing sign for small positive $l$ and becoming increasingly negative for increasing $\omega$. Increasing $T_{f}$ increases the slope of the $l$ phase near the zero crossing and shifts the $\omega$ phase toward larger negative values over all $\omega$.

\section{Discussion}

Fluctuations in alongshore wind stress $\tau$ calculated from FNOC geostrophic winds along the west coast of North America are relatively energetic at alongshore wavenumbers $|l| \leq 1.1 \times 10^{-3} \mathrm{cpkm}$ and frequencies $0.025<\omega<0.5 \mathrm{cpd}$, and they effectively drive a coastal sea level $\zeta$ response within this $(l, \omega)$ band. Winter $\tau$ fluctuations are generated primarily by spatially coherent, propagating weather systems [Halliwell and Allen, 1987], and equatorward propagation dominates these fluctuations for $\omega<0.35 \mathrm{cpd}$. Poleward propagation strongly dominates the summer $\tau$ fluctuations for $\omega<0.1 \mathrm{cpd}$, and equatorward propagating variance slightly exceeds poleward propagating variance for larger $\omega$. In contrast, poleward propagation dominates the $\zeta$ fluctuations in both seasons, but more so in summer. The $\zeta$ response at a given location is most highly correlated with $\tau$ time series at lag distances $300-400$ $\mathrm{km}$ equatorward of that location. Coherence analysis indicates that the lag distance is approximately $-360 \mathrm{~km}$ for $\omega \leq 0.11$ 
cpd $(\omega<0.19 \mathrm{cpd})$ in summer (winter), and is approximately $-180 \mathrm{~km}$ for larger $\omega$ in both seasons, except near $\omega=0.34$ cpd in summer, where it is approximately 0 .

The largest coherence between $\zeta$ and $\tau$ as a function of $(l, \omega)$ is observed where $\tau$ is most energetic, i.e., for positive $l$ in summer and negative $l$ in winter. The $\zeta$ response to $\tau$ is apparently very weak for $|l|>1.1 \times 10^{-3} \mathrm{cp} \mathrm{km}$ in both seasons since the coherence is small for all $\omega$ at these large $l$.. This result is not an artifact of using calculated $\tau$ as the forcing function, because similar results are obtained using measured $\tau$. The observed transfer functions indicate that a resonance exists in both seasons, with the largest gain occurring along a single ridge that approximately follows a straight line (dispersion curve) of constant poleward phase speed, and with $\zeta$ approximately in phase with $\tau$ along this ridge. The gain along this ridge decreases substantially with increasing $l$ and $\omega$ in both seasons, with the largest response confined to even smaller $l$ and $\omega$ in winter than in summer. The decrease is also not an artifact of using calculated $\tau$ as the forcing function, since it is also present, and even tends to be slightly larger, when measured $\tau$ is used. The interannual variability in this observed pattern of gain is large, with only small decreases in maximum gain observed during some seasons and very large decreases observed in others. The phase patterns are more similar among individual summer and winter seasons.

The $\zeta$ response is more effectively forced by $\tau$ in summer than in winter. The ratio of total $\zeta$ variance to total $\tau$ variance is nearly 4 times greater in summer. Two factors are evidently responsible for this: First, the magnitude of the gain is substantially smaller in winter than in summer, which is probably due primarily to larger friction in winter. Second, the dominance of equatorward propagation in the winter $\tau$ field causes substantially more $\tau$ variance to exist in a region of $(l, \omega)$ space away from the dispersion curve in locations where the gain is relatively small.

A single forced, first-order wave equation model with a linear friction term governing the $(y, t)$ dependence of the response due to one coastally trapped wave mode predicts that a resonant response exists. A constant maximum in the predicted gain follows the straight dispersion curve $c=\omega / l>0$ in $(l, \omega)$ space, and the predicted phase lag of the response is zero along this curve. These predicted patterns are qualitatively consistent with the observed patterns, except for the observed deceased in maximum gain with increasing $l$ and (1) along the dispersion curve. Relatively good agreement exists between observed and predicted phases. Since this decrease in gain is not predicted by one wave equation, we attempted to identify possible causes. Alongshore variations in the parameters for one wave equation (phase speed, friction time scale, and coupling coefficient) did not distort the predicted gain and phase patterns very much. Models with $(l, \omega)$-dependent friction did not successfully predict properties of the observed transfer functions. A significant contribution to the response from additional coastally trapped wave modes can incease the predicted maximum gain at small $l$ and $w$. This occurs because the individual ridges of large gain merge and become indistinguishable at small $l$ and $\omega$, and within this part of $(l, \omega)$ space, the gain decreases with increasing $l$ and $\omega$ as observed. The presence of higher modes may therefore contribute to the observed decrease. The possible existence of frictional coupling among the first several modes does not contribute to the observed decrease.

\section{Appendix A: Space-Lagged Correlation, Coherence, and Phase Functions}

Given a set of $N$ evenly spaced time series sampled during $M$ seasons, the space-lagged autocorrelation function for the $\tau$ field $\left(\hat{R}_{\tau \tau}\right)$ and the space-lagged cross-correlation function between the $\tau$ and $\zeta$ fields $\left(\hat{R}_{\zeta \tau}\right)$, both seasonally averaged over the $M$ seasons, are calculated, respectively, as follows:

$$
\begin{aligned}
& \hat{R}_{\tau \tau}\left(\eta_{n}, t_{l}\right) \\
& =\frac{\sum_{m=1}^{M} \sum_{i=I}^{J}\left\langle\tau\left(y_{t}, t\right) \tau\left(y_{t}+\eta_{n}, t+t_{l}\right)\right\rangle}{\left[\sum_{m=1}^{M} \sum_{i=I}^{J}\left\langle\tau^{2}\left(y_{i}, t\right)\right\rangle\right]^{1 / 2}\left[\sum_{m=1}^{M} \sum_{i=I}^{J}\left\langle\tau^{2}\left(y_{t}+\eta_{n}, t+t_{l}\right)\right\rangle\right]^{1 / 2}}
\end{aligned}
$$

$\hat{R}_{\zeta \tau}\left(\eta_{n}, t_{l}\right)$

$$
=\frac{\sum_{m=1}^{M} \sum_{t=I}^{J}\left\langle\zeta\left(y_{i}, t\right) \tau\left(y_{i}+\eta_{m}, t+t_{t}\right)\right\rangle}{\left[\sum_{m=1}^{M} \sum_{i=1}^{J}\left\langle\zeta^{2}\left(y_{l}, t\right)\right\rangle\right]^{1 / 2}\left[\sum_{m=1}^{M} \sum_{i=I}^{J}\left\langle\tau^{2}\left(y_{i}+\eta_{n}, t+t_{t}\right)\right\rangle\right]^{1 / 2}}
$$

where

$$
\left\{\begin{array}{ll}
I=1 & J=N-n \\
I=|n|+1 & J=N
\end{array}\right\} \text { for }\left\{\begin{array}{ll}
\quad \begin{array}{l}
n \geq 0 \\
n<0
\end{array} & |n| \leq|N-1|
\end{array}\right\}
$$

and where the circumflex denotes statistics estimated from the data. The autocorrelation function for the $\zeta$ field, $\hat{R}_{\zeta \zeta}\left(\eta_{n}, t_{l}\right)$, is calculated in the same manner as $\hat{R}_{\tau \tau}\left(\eta_{n}, t_{l}\right)$ using $\zeta$ in place of $\tau$ in (A1). The angle brackets signify time averaging, and the mean values of $\tau$ and $\zeta$ are assumed to be removed. The functions $\hat{R}_{\tau \tau}, \hat{R}_{\zeta \zeta}$, and $\hat{R}_{\zeta \tau}$ are contoured in Figure 4 . We estimate integral correlation space scales of $\tau$ and $\zeta$ by integrating $\hat{R}_{\mathrm{rr}}\left(\eta_{n}, 0\right)$ and $\hat{R}_{\zeta \zeta}\left(\eta_{n}, 0\right)$ over positive $\eta_{n}$ out to the first zero crossing of $\hat{R}$. We estimate correlation time scales of $\tau$ and $\zeta$ by integrating $\hat{R}_{\tau \tau}\left(0, t_{l}\right)$ and $\hat{R}_{\zeta \zeta}\left(0, t_{l}\right)$ over positive $t_{l}$ out to the first zero crossing of $\hat{R}$. In estimating these scales, we integrated out to space or time lags larger than the maximum lags shown in Figure 4, if necessary. In winter, the zero crossing of $\hat{R}_{\zeta \zeta}\left(\eta_{n}, 0\right)$ is not reached by the maximum space lag that we integrated to $\left(\eta_{n}=2520 \mathrm{~km}\right)$, but $\hat{R}_{\zeta \zeta}\left(\eta_{n}, 0\right)$ has decreased to less than 0.1 by this lag. If $\hat{R}_{\zeta \zeta}\left(\eta_{n}, 0\right)$ is assumed to decrease linearly beyond this maximum lag, then the winter correlation space scale for $\zeta$ is underestimated by no more than $5 \%$. All space and time scales are summarized in Table 4.

The seasonally averaged space-lagged coherence squared functions for the $\tau$ field, and between the $\zeta$ and $\tau$ fields, are given by

$$
\begin{aligned}
\hat{i}_{\tau \tau}^{2}\left(\eta_{n}, \omega\right)= & \left|\sum_{m=1}^{M} \sum_{i=I}^{J}\left\{\bar{\tau}^{*}\left(y_{i}, \omega\right) \bar{\tau}\left(y_{1}+\eta_{m}, \omega\right)\right\}\right|^{2} \\
& \cdot\left\{\left[\sum_{m=1}^{M} \sum_{i=I}^{J}\left\{\bar{\tau}^{*}\left(y_{l}, \omega\right) \bar{\tau}\left(y_{l}, \omega\right)\right\}\right]\right. \\
& \left.\cdot\left[\sum_{m=1}^{M} \sum_{i=I}^{J}\left\{\bar{\tau}^{*}\left(y_{1}+\eta_{n}, \omega\right) \bar{\tau}\left(y_{1}+\eta_{n}, \omega\right)\right\}\right]\right\}^{-1}
\end{aligned}
$$




$$
\begin{aligned}
\hat{\gamma}_{\zeta \tau}{ }^{2}\left(\eta_{n}, \omega\right)= & \left|\sum_{m=1}^{M} \sum_{i=I}^{J}\left\{\bar{\zeta}^{*}\left(y_{i}, \omega\right) \bar{\tau}\left(y_{i}+\eta_{n}, \omega\right)\right\}\right|^{2} \\
& \cdot\left\{\left[\sum_{m=1}^{M} \sum_{i=I}^{J}\left\{\bar{\zeta}^{*}\left(y_{i}, \omega\right) \bar{\zeta}\left(y_{i}, \omega\right)\right\}\right]\right. \\
& \left.\cdot\left[\sum_{m=1}^{M} \sum_{i=I}^{J}\left\{\bar{\tau}^{*}\left(y_{i}+\eta_{n}, \omega\right) \bar{\tau}\left(y_{i}+\eta_{m}, \omega\right)\right\}\right]\right\}^{-1}
\end{aligned}
$$

where $I$ and $J$ are computed using (A3). The overbar denotes the Fourier transform, an asterisk denotes complex conjugate, and braces denote frequency band averaging. The coherence squared for the $\zeta$ field is calculated in the same manner as for the $\tau$ field using (A4). The phase functions for the $\tau$ field and between the $\tau$ and $\zeta$ fields, respectively, are given by

$$
\begin{aligned}
& \hat{\theta}_{\tau \tau}\left(\eta_{n}, \omega\right)=\arg \left(\sum_{m=1}^{M} \sum_{i=I}^{J}\left\{\bar{\tau}^{*}\left(y_{i}, \omega\right) \bar{\tau}\left(y_{t}+\eta_{n}, \omega\right)\right\}\right) \\
& \hat{\theta}_{\zeta \tau}\left(\eta_{n}, \omega\right)=\arg \left(\sum_{m=1}^{M} \sum_{i=I}^{J}\left\{\bar{\zeta}^{*}\left(y_{i}, \omega\right) \bar{\tau}\left(y_{i}+\eta_{n}, \omega\right)\right\}\right)
\end{aligned}
$$

With $\hat{\theta}$ in radians, the phase speed function for $\tau$ is given by

$$
\hat{c}_{\tau \tau}\left(\eta_{n}, \omega\right)=\frac{\omega \eta_{n}}{\hat{\theta}_{\tau \tau}\left(\eta_{n}, \omega\right)}
$$

We band average over nine adjacent frequency bands, excluding $\omega=0$, and ensemble average over four seasons, which would result in estimates with 72 degrees of freedom if no spatial averaging were performed. The spatial averaging will increase the number of degrees of freedom by an amount that is a function of $\eta_{n}$. The increase will be largest for $\eta_{n}=0$, where the spectrum estimates are spatially averaged over 2-3 spatial correlation scales (section 3.2). At the largest space lags, the spatial averaging is only over one correlation space scale, so no additional degrees of freedom are gained. We therefore assume the estimates have $\mathbf{7 2}$ degrees of freedom, for which the minimum significant coherence squared is 0.041 [Thompson, 1979].

\section{Appendix B: Calculation of the $(l, \omega)$ Spectrum EsTIMATES}

We use the Fourier transform method in two dimensions to estimate the $(l, \omega)$ autospectra and cross-spectra. Given the discrete data sets $\tau\left(y_{j}, t_{k}\right)$ and $\zeta\left(y_{j}, t_{k}\right)$ for a given season sampled at $N$ points along the coast and $L$ points in time with sampling rates $\Delta y$ and $\Delta t$, raw (unaveraged) estimates of the autospectra of $\tau$ and $\zeta$, and the cross-spectrum between $\tau$ and $\zeta$, are given by

$$
\begin{aligned}
& \hat{S}_{\tau}\left(l_{m}, \omega_{n}\right)=B_{l} B_{\omega} \bar{\tau}^{*}\left(l_{m}, \omega_{n}\right) \bar{\tau}\left(l_{m}, \omega_{n}\right) \\
& \hat{S}_{\zeta}\left(l_{m}, \omega_{n}\right)=B_{l} B_{\omega} \bar{\zeta}^{*}\left(l_{m}, \omega_{n}\right) \bar{\zeta}\left(l_{m}, \omega_{n}\right) \\
& \hat{S}_{\tau \zeta}\left(l_{m}, \omega_{n}\right)=B_{l} B_{\omega} \bar{\tau}^{*}\left(l_{m}, \omega_{n}\right) \bar{\zeta}\left(l_{m}, \omega_{n}\right)
\end{aligned}
$$

where the overbar denotes a two-dimensional Fourier transform, the asterisk denotes complex conjugate, and where $B_{l}=$ $(N \Delta y)^{-1}$ and $B_{\omega}=(L \Delta t)^{-1}$ are the wave number and frequency bandwidths respectively, for the raw estimates. The discrete two-dimensional Fourier transform of a data matrix $f\left(y_{j}, t_{k}\right)$ is

$$
\bar{f}\left(l_{m}, \omega_{n}\right)=\Delta y \Delta t \sum_{j=1}^{N} \sum_{k=1}^{L} f\left(y_{j}, t_{k}\right) e^{i\left(l_{n} y,-\omega_{n} t_{k}\right)}
$$

where the discrete wave numbers and frequencies of the raw spectrum estimates are given by

$$
\left\{\begin{array}{l}
l_{m}=m(N \Delta y)^{-1} \\
\omega_{n}=n(L \Delta t)^{-1}
\end{array}\right\} \text { for } \quad\left\{\begin{array}{c}
-N / 2<m<N / 2 \\
0<n<L / 2
\end{array}\right\}
$$

Spectrum estimates at negative frequencies contain redundant information.

The raw spectrum estimates are $\chi^{2}$ random variables with approximately 2 degrees of freedom. We must therefore average these estimates to increase their statistical stability. The averaged estimates are also $\chi^{2}$ random variables, but with $2 N_{\mathrm{s}}$ degrees of freedom, where $N_{s}$ is the total number of raw estimates that are averaged [Bendat and Piersol, 1971]. The raw estimates may be band averaged over wave number, band averaged over frequency, and/or ensemble averaged over two or more realizations (or in our case, seasons). The number of degrees of freedom will then be given by

$$
n_{\text {dof }}=2 n_{l} n_{\omega} n_{r}
$$

where $n_{l}$ is the number of wave number bands, $n_{\omega}$ is the number of frequency bands, and $n_{r}$ is the number of realizations over which spectrum estimates are averaged. Because of limited resolution, we do not band average over wave number. To generate the average summer and winter spectrum estimates, we ensemble average over four seasons.

We also corrected for leakage effects by prewhitening and postcoloring. Frankignoul [1974] noted that if a time series was relatively short compared to the dominant period of an energetic signal in the data, leakage could substantially distort the calculated autospectrum function at higher frequencies. If the series is long compared to this strong signal, leakage effects will be small, and the calculated spectrum will approximate the shape of the true spectrum. As the length of the series decreases, the slope of the calculated spectrum will asymptotically approach $\omega^{-2}$ as the length becomes of the same order as or smaller than the period of the strong signal, no matter what the slope of the true spectrum is. We were therefore concerned that this could be a problem in the wave number domain, since the length of the domain is comparable to the horizontal scales of synoptic atmospheric systems (cyclones and anticyclones). This concern was heightened because the initial wave number autospectra of both $\tau$ and $\zeta$ that we calculated had slopes close to $l^{-2}$ in most of the wave number domain that we resolved. Because of this, we prewhitened $\tau$ and $\zeta$ in the alongshore domain only using the first-difference method prior to calculating spectrum functions, and then postcolored these functions in the wave number domain, as described by Frankignoul [1974] for frequency domain spectrum functions. We tested how prewhitening in the space domain affected the shapes of wave number autospectra of both $\tau$ and $\zeta$ and found that it made little difference. (Prewhitening was not performed in the $(l, \omega)$ spectrum analyses of the wind fields presented by Halliwell and Allen [1987], but test calculations indicate that prewhitening had very minor effects on these spectra.) Apparently, the slopes of the true spectrum functions are close to $l^{-2}$ in most of the wave number domain. We also tested if prewhitening in the time domain had a significant effect on frequency spectra. Since the time series were long with respect to the periods of synoptic wind fluctuations, we did not expect frequency domain leakage to be a serious problem, and this was confirmed by the tests. (We also note that the one-dimensional spectrum functions in Figures 26 
and 27 were computed as shown in Appendix $\mathrm{C}$ using $(l, \omega)$ autospectra that were not prewhitened and postcolored.)

The coherence squared and transfer functions are given by

$$
\begin{gathered}
\hat{\gamma}_{\tau \zeta}^{2}\left(l_{m}, \omega_{n}\right)=\frac{\left|\left\{\hat{S}_{\tau \zeta}\left(l_{m}, \omega_{n}\right)\right\}\right|^{2}}{\left\{\hat{S}_{\tau}\left(l_{m}, \omega_{n}\right)\right\}\left\{\hat{S}_{\zeta}\left(l_{m}, \omega_{n}\right)\right\}} \\
H_{\tau \zeta}\left(l_{m}, \omega_{n}\right)=\frac{\left\{\hat{S}_{\tau \zeta}\left(l_{m}, \omega_{n}\right)\right\}}{\hat{S}_{\tau}\left(l_{m}, \omega_{n}\right)}
\end{gathered}
$$

where the braces denote the averaging performed. The gain and phase of the transfer functions are given by

$$
\begin{gathered}
\hat{G}_{\tau \zeta}\left(l_{m}, \omega_{n}\right)=\left|H_{\tau \zeta}\left(l_{m}, \omega_{n}\right)\right| \\
\hat{\theta}_{\tau \zeta}\left(l_{m}, \omega_{n}\right)=\arg \left[H_{\tau \zeta}\left(l_{m}, \omega_{n}\right)\right]
\end{gathered}
$$

\section{Appendix C: $\zeta$ Response Predicted by Forced, First-Order Wave Equations}

The forced, first-order wave equation with a linear friction term describing the alongshore-time dependence of the $\zeta$ response to $\tau$ is

$$
c_{n}{ }^{-1} \zeta_{n t}+\zeta_{n y}+\left(c_{n} T_{f n}\right)^{-1} \zeta_{n}=b_{n} \tau
$$

where $c_{n}$ are the free-wave phase speeds, $T_{f n}$ are the friction time scales, and $b_{n}$ are the coupling coefficients for the $\zeta_{n}$ response. Using the continuous two-dimensional Fourier transform

$$
\bar{f}(l, \omega)=\int_{-\infty}^{\infty} \int_{-\infty}^{\infty} f(y, t) e^{(l y-\omega t)} d y d t
$$

assuming that the first mode dominates the response (dropping the $n=1$ subscript for simplicity), and assuming constant coeflicients, we obtained the transformed wave equation

$$
\left[i \omega c^{-1}-i l+\left(c T_{f}\right)^{-1}\right] \bar{\zeta}=b \bar{\tau}
$$

The predicted transfer function $H_{\tau \zeta}(l, \omega)$ is therefore

$$
H_{\tau \zeta}(l, \omega)=\frac{c T_{f} b}{1-i T_{f}(l c-\omega)}=G_{\tau \zeta} e^{i \theta_{\tau \zeta}}
$$

with the gain $G_{\tau \zeta}$ and phase $\theta_{\tau \zeta}$ given by

$$
\begin{gathered}
G_{\tau \zeta}(l, \omega)=\frac{c T_{f} b}{\left[1+T_{f}{ }^{2}(l c-\omega)^{2}\right]^{1 / 2}} \\
\theta_{\tau \zeta}(l, \omega)=\tan ^{-1}\left[T_{f}(l c-\omega)\right]
\end{gathered}
$$

If additional modes contribute significantly to the response, the transfer function of the total response equals the sum of the transfer functions of the individual modes. If two modes dominate the response, then $\zeta=\zeta_{1}+\zeta_{2}$, and

$$
\begin{aligned}
H_{\tau \zeta}(l, \omega) & =\left\{b_{1}\left[i\left(\omega c_{2}{ }^{-1}-l\right)+\left(c_{2} T_{f 2}\right)^{-1}\right]\right. \\
+ & \left.b_{2}\left[i\left(\omega c_{1}{ }^{-1}-l\right)+\left(c_{1} T_{f 1}\right)^{-1}\right]\right\}\left\{\left[i\left(\omega c_{1}{ }^{-1}-l\right)\right.\right. \\
+ & \left.\left.\left(c_{1} T_{f 1}\right)^{-1}\right]+\left[i\left(\omega c_{2}{ }^{-1}-l\right)+\left(c_{2} T_{f 2}\right)^{-1}\right]\right\}^{-1}
\end{aligned}
$$

If friction is sufficiently strong, the modes can be frictionally coupled to each other [Brink, 1982; Chapman, 1987], and the $(y-t)$ dependence of the $\zeta$ response is described by an infinite set of coupled wave equations:

$$
c_{n}{ }^{-1} \zeta_{n t}+\zeta_{n y}+\sum_{m=1}^{\infty} a_{n m} \zeta_{m}=b_{n} \tau
$$

The diagonal elements of the frictional coeflicient matrix are given by

$$
a_{n n}=\left(c_{n} T_{f n}\right)^{-1}
$$

and the off-diagonal elements of $a_{n m}$ couple mode $n$ to mode $m$. If two modes dominate the response, the Fourier transforms of the coupled equations are

$$
\begin{aligned}
& {\left[i\left(\omega c_{1}^{-1}-l\right)+a_{11}\right] \bar{\zeta}_{1}+a_{12} \bar{\zeta}_{2}=b_{1} \tau} \\
& a_{21} \bar{\zeta}_{1}+\left[i\left(\omega c_{2}{ }^{-1}-l\right)+a_{22}\right] \bar{\zeta}_{2}=b_{2} \tau
\end{aligned}
$$

By solving this system for $\bar{\zeta}_{1}, \bar{\zeta}_{2}$ and adding, the total transfer function is found to be

$$
\begin{aligned}
H_{\tau \zeta}(l, \omega)= & \left\{b_{1}\left[i\left(\omega c_{2}^{-1}-l\right)+a_{22}\right]\right. \\
& +b_{2}\left[i\left(\omega c_{1}^{-1}-l\right)+a_{11}\right]-b_{1} a_{21} \\
& \left.-b_{2} a_{12}\right\}\left\{\left[i\left(\omega c_{1}{ }^{-1}-l\right)+a_{11}\right]\right. \\
& \left.+\left[i\left(\omega c_{2}^{-1}-l\right)+a_{22}\right]-a_{12} a_{21}\right\}^{-1}
\end{aligned}
$$

By comparing (C11) to (C7), the effects of mode coupling are evident. In our tests of the effects of mode coupling, we estimated the off-diagonal elements of the frictional coefficient matrix using

$$
a_{n m}=\beta a_{\mathrm{nn}} \quad m \neq n
$$

We then calculated transfer functions for several values of $\beta$ to assess how it was affected by varying the strength of the coupling.

Given the autospectrum of $\tau, S_{\tau}(l, \omega)$, then the predicted cross-spectrum between $\tau$ and $\zeta, S_{\tau \zeta}(l, \omega)$ and the predicted autospectrum of $\zeta, S_{\zeta}(l, \omega)$, are given by

$$
\begin{gathered}
S_{\tau \zeta}(l, \omega)=H_{\tau \zeta}(l, \omega) S_{\tau}(l, \omega) \\
S_{\zeta}(l, \omega)=\left|H_{\tau \zeta}(l, \omega)\right|^{2} S_{\tau}(l, \omega)
\end{gathered}
$$

The coherence squared is

$$
\gamma_{\tau \zeta}^{2}(l, \omega)=\frac{\left|S_{\tau \zeta}(l, \omega)\right|^{2}}{S_{\tau}(l, \omega) S_{\zeta}(l, \omega)}=1
$$

The coherence squared and transfer functions can be calculated as functions of $l$ or $\omega$ only, and we describe these calculations here for functions of $l$. Given

$$
S_{\tau}(l)=\int_{0}^{\infty} S_{\tau}(l, \omega) d \omega
$$

we can calculate $S_{\tau \zeta}(l)$ and $S_{\zeta}(l)$ by integrating $(\mathrm{Cl} 3)$ and $(\mathrm{Cl} 14)$ over $\omega$, as was done for $S_{\tau}(l)$ in $(\mathrm{C} 16)$, and then calculate the $l$ coherence squared and transfer functions by

$$
\begin{gathered}
\gamma_{\mathrm{\tau} \zeta}^{2}(l)=\frac{\left|S_{\tau \zeta}(l)\right|^{2}}{S_{\mathrm{\tau}}(l) S_{\zeta}(l)} \\
H(l)=\frac{S_{\tau \zeta}(l)}{S_{\tau}(l)}
\end{gathered}
$$

The maximum achievable coherence squared, $\gamma_{r \zeta}{ }^{2}(l)$, is generally less than 1 . To calculate the one-dimensional coherence squared and transfer functions presented in section 6.3 , we numerically integrate $(\mathrm{C} 13)$ and $(\mathrm{C} 14)$ over $\omega$ and numerically calculate the integral in (C16) using $(\mathrm{C} 4)$ and observed values of $S_{\tau}(l, \omega)$ (Figure 7). We then calculate $\gamma_{\tau \zeta}{ }^{2}$ and $\theta_{\tau \zeta}$ using (C17) 
and (C18). The numerical integrations are performed using the trapezoidal rule.

\section{Appendix D: Effects of Noise on $(l, \omega)$ Coherence and Transfer Function Estimates}

Assume that the output function $\zeta(y, t)$ is related to the input function $\tau(y, t)$ through a two-dimensional linear model

$$
L \zeta(y, t)=b \tau(y, t)
$$

where $L$ is a linear operator. If all terms of (D1) have constant coefficients, it can be Fourier transformed using (C2) to yield

$$
\bar{\zeta}(l, \omega)=H(l, \omega) \bar{\tau}(l, \omega)
$$

where $H(l, \omega)$ is the transfer function of the model. In general, when we estimate a transfer function using discrete samples of $\zeta\left(y_{j}, t_{k}\right)$ and $\tau\left(y_{j}, t_{k}\right)$, noise will be present in these samples, and we must understand how this noise affects the estimated transfer function $\hat{H}\left(l_{m}, \omega_{n}\right)$ before we can compare it to the model transfer function $H(l, \omega)$.

We assume that the sampled data sets $\tau\left(y_{j}, t_{k}\right)$ and $\zeta\left(y_{j}, t_{k}\right)$ consist of a signal plus noise. Here we define noise as all variance in each data set that is not explained by the model. This includes random noise in both $\tau$ and $\zeta$, but also includes $\zeta$ variance that is forced by other processes. In our analyses, for example, this may include free-wave energy propagating poleward from the equatorial region that is incoherent with $\tau$ within our analysis domain. Fourier transforming $\tau\left(y_{j}, t_{k}\right)$ and $\zeta\left(y_{j}, t_{k}\right)$ using (B4) therefore yields

$$
\begin{aligned}
& \bar{\tau}=\bar{\tau}_{s}+\bar{\tau}_{e} \\
& \bar{\zeta}=\bar{\zeta}_{s}+\bar{\zeta}_{e}
\end{aligned}
$$

where all transformed variables are functions of $\left(l_{m}, \omega_{n}\right)$, and where the subscript $s$ denotes signal and $e$ denotes noise. The transforms of the signals are related by the linear model (D2):

$$
\bar{\zeta}_{s}=H_{\tau \zeta} \bar{\tau}_{s}
$$

We now show how noise can cause the observed transfer function $\hat{H}_{\tau \zeta}$ to differ from the model transfer function $H_{\tau \zeta}$. We define the estimated spectrum functions:

$$
\begin{aligned}
& \hat{S_{\tau}}=\left\{\bar{\tau}^{*} \bar{\tau}\right\}=\left\{\left(\bar{\tau}_{s}+\bar{\tau}_{e}\right)^{*}\left(\bar{\tau}_{s}+\bar{\tau}_{e}\right)\right\} \\
& \hat{S}_{\zeta}=\{\bar{\zeta} * \bar{\zeta}\}=\left\{\left(\bar{\zeta}_{s}+\bar{\zeta}_{e}\right)^{*}\left(\bar{\zeta}_{s}+\bar{\zeta}_{e}\right)\right\} \\
& \hat{S}_{\tau \zeta}=\left\{\bar{\tau}^{*} \bar{\zeta}\right\}=\left\{\left(\bar{\tau}_{s}+\bar{\zeta}_{e}\right)^{*}\left(\bar{\tau}_{s}+\bar{\zeta}_{e}\right)\right\}
\end{aligned}
$$

If we assume that $\tau_{e}$ and $\zeta_{e}$ are incoherent with each other and with the signals, then using (D4) we obtain

$$
\begin{gathered}
\hat{S}_{\tau}=\left\{\bar{\tau}_{s}{ }^{*} \bar{\tau}_{s}\right\}+\left\{\bar{\tau}_{e}{ }^{*} \bar{\tau}_{e}\right\} \\
\hat{S}_{\zeta}=\left|H_{\tau \zeta}\right|^{2}\left\{\bar{\tau}_{s}{ }^{*} \bar{\tau}_{s}\right\}+\left\{\bar{\zeta}_{e}{ }^{*} \bar{\zeta}_{e}\right\} \\
\hat{S}_{\tau \zeta}=\left|H_{\tau \zeta}\right|\left\{\bar{\tau}_{s}^{*} \bar{\tau}_{s}\right\}
\end{gathered}
$$

The observed coherence squared and transfer functions are

$$
\begin{gathered}
\hat{\gamma}_{\tau \zeta}{ }^{2}=\frac{\left|\hat{S}_{\tau \zeta}\right|^{2}}{\hat{S}_{\tau} \hat{S}_{\zeta}}=\frac{\mid H_{\tau \zeta}{ }^{2}\left\{\bar{\tau}_{s}^{*} \bar{\tau}_{s}\right\}^{2}}{\left[\left\{\bar{\tau}_{s}^{*} \bar{\tau}_{s}\right\}+\left\{\bar{\tau}_{e}^{*} \bar{\tau}_{e}\right\}\right]\left[\left|H_{\tau \zeta}\right|^{2}\left\{\bar{\tau}_{s}^{*} \bar{\tau}_{s}\right\}+\left\{\bar{\zeta}_{e}^{*} \bar{\zeta}_{e}\right\}\right]} \\
\hat{H}_{\tau \zeta}=\frac{\hat{S}_{\tau \zeta}}{\hat{S}_{\tau}}=\frac{H_{\tau \zeta}\left\{\bar{\tau}_{s}^{*} \bar{\tau}_{s}\right\}}{\left[\left\{\bar{\tau}_{s}{ }^{*} \bar{\tau}_{s}\right\}+\left\{\bar{\tau}_{e}{ }^{*} \bar{\tau}_{e}\right\}\right]}
\end{gathered}
$$

with the gain and phase given by

$$
\begin{gathered}
\hat{G}_{\tau \zeta}=\left|\hat{H}_{\tau \zeta}\right|=\left|H_{\tau \zeta}\right|\left(\frac{\left\{\bar{\tau}_{s}^{*} \bar{\tau}_{s}\right\}}{\left[\left\{\bar{\tau}_{s}^{*} \bar{\tau}_{s}\right\}+\left\{\bar{\tau}_{e}{ }^{*} \bar{\tau}_{e}\right\}\right]}\right) \\
\hat{\theta}=\arg \left(\hat{H}_{\tau \zeta}\right)=\arg \left(H_{\tau \zeta}\right)
\end{gathered}
$$

Therefore noise in the $\tau$ and $\zeta$ data sets has the following influence on the observed coherence, gain, and phase functions: (1) In the absence of noise in both data sets, $\hat{\gamma}^{2}$ would equal 1 for all $l_{m}$ and $\omega_{n}$. The presence of noise in either data set will cause $\hat{\gamma}^{2}$ to decrease. (2) The presence of noise in only the input $(\tau)$ data set will cause $\hat{G}_{\tau \zeta}=\left|\hat{H}_{\tau \zeta}\right|$ to be smaller than the true model gain $G_{\tau \zeta}=\left|H_{\tau \zeta}\right|$. (3) The value of $\hat{\theta}$ is not affected by noise in either the input or output data set. Therefore $\theta$ is an unbiased estimator of $\theta$, but $\hat{G}_{\tau \zeta}$ will be too small at all $\left(l_{m}, \omega_{n}\right)$ where the input $\tau$ data set has a poor signal-tonoise ratio.

Acknowledgments. This research was performed with the support of the Oceanography section of the National Science Foundation under grant OCE-8411613 as part of the Coastal Ocean Dynamics Experiment (CODE). We express our gratitude to the organizations and people that have provided the wind data used in the study. We especially thank Dudley Chelton for discussions on prewhitening and postcoloring the time series. We also thank Andrew Bakun for help in obtaining the calculated wind set and Henry Pittock for processing coastal sea level data. All figures (except for the shading) were generated using the interactive graphics package PLOT5, developed by Donald W. Denbo. CODE contribution 55.

\section{REFERENCES}

Allen, J. S., Models of wind-driven currents on the continental shelf, Annu. Rev. Fluid Mech., 12, 389-434, 1980.

Allen, J. S., A simple model for stratified shelf flow fields with bottom friction, J. Phys. Oceanogr., 14, 1200-1214, 1984.

Allen, J. S., and D. W. Denbo, Statistical characteristics of the largescale response of coastal sea level to atmospheric forcing, $J$. Phys. Oceanogr., 14, 1079-1094, 1984.

Bakun, A., Daily and weekly upwelling indices, west coast of North America, 1967-73, NOAA Tech. Rep. NMFS SSRF-693, 114 pp., 1975.

Bendat, J. S., and A. C. Piersol, Random Data: Analysis and Measurement Techniques, 407 pp., John Wiley, New York, 1971.

Brink, K. H., The effect of bottom friction on low frequency coastal trapped waves, J. Phys. Oceanogr., I2, 127-133, 1982.

Brink, K. H., D. C. Chapman, and G. R. Halliwell, Jr., A stochastic model for wind-driven currents over the continental shelf, J. Geophys. Res., 92, 1783-1797, 1987.

Chapman, D. C., Application of wind-forced, long, coastal-trapped wave theory along the California coast, J. Geophys. Res., 92, 1798$1816,1987$.

Clarke, A. J., Observational and numerical evidence for wind-forced coastal trapped long waves, J. Phys. Oceanogr., 7, 231-247, 1977.

Dorman, C. E., Evidence of Kelvin waves in California's marine layer and related eddy generation, Mon. Weather Rev., 113, 827-839, 1985.

Dorman, C. E., Possible role of gravity currents in Northern California's coastal summer wind reversals, $J$. Geophys. Res., 92, 1497$1506,1987$.

Dorr, A., and R. Grimshaw, Barotropic continental shelf waves on a $\beta$ plane, J. Phys. Oceanogr., 16, 1345-1358, 1986.

Frankignoul, C., A cautionary note on the spectral analysis of short internal wave records, J. Geophys. Res., 79, 3459-3462, 1974.

Gill, A. E., and A. J. Clarke, Wind-induced upwelling, coastal currents, and sea level changes, Deep Sea Res., 21, 325-345, 1974.

Gill, A. E., and E. H. Schumann, The generation of long shelf waves by the wind, J. Phys. Oceanogr., 4, 83-90, 1974.

Grant, W. D., and O. S. Madsen, Combined wave and current interaction with a rough bottom, J. Geophys. Res., 84, 1797-1808, 1979.

Halliwell, G. R., Jr., and J. S. Allen, CODE-1: Large-scale wind and sea level observations, CODE-1: Moored Array and Large-Scale Data Report, WHOI Tech. Rep. 82-23, edited by R. C. Beardsley 
and L. K. Rosenfeld, pp. 139-185, Woods Hole Oceanogr. Inst., Woods Hole, Mass., 1983.

Halliwell, G. R., Jr., and J. S. Allen, Large-scale sea level response to atmospheric forcing along the west coast of North America summer 1973, J. Phys. Oceanogr., 14, 864-886, 1984.

Halliwell, G. R., Jr., and J. S. Allen, CODE-2: Large-scale wind and sea level observations, CODE-2: Moored Array and Large-Scale Data Report, WHOI Tech. Rep. 85-35, edited by R. Limeburner, pp. 179-233, Woods Hole Oceanogr. Inst., Woods Hole, Mass., 1985.

Halliwell, G. R., Jr., and J. S. Allen, The large-scale coastal wind field along the west coast of North America, 1981-1982, J. Geophys. Res., 92, 1861-1884, 1987.

Halliwell, G. R., Jr., H. L. Pittock, V. M. Halliwell, and J. S. Allen, The CODE large-scale meteorological, sea surface temperature, and coastal sea level data set, 1980-84, Ref. 86-15, Data Rep. 125, 442 pp., Coll. of Oceanogr., Oreg. State Univ., Corvallis, 1986.

Hamon, B. V., Generation of shelf waves on the east Australian coast by wind stress, Mem. Soc. R. Sci. Liege, 10, 359-367, 1976.

Large, W. G., and S. Pond, Open ocean flux measurements in moderate to strong winds, J. Phys. Oceanogr., 11, 324-336, 1981.

Nelson, C. S., Wind stress and wind stress curl over the California Current, NOAA Tech. Rep. SSRF-714, 87 pp., 1977.
Pittock, H. L., W. E. Gilbert, A. Huyer, and R. L. Smith, Observations of sea level, wind, and atmospheric pressure at Newport, Oregon, 1967-1980, Data Rep. 98, 158 pp., Oreg. State Univ., Corvallis, 1982.

Strub, P. T., J. S. Allen, A. Huyer, and R. L. Smith, Large-scale structure of the spring transition in the coastal ocean off western North America, J. Geophys. Res., 92, 1527-1544, 1987.

Thompson, R. O. R. Y., Coherence significance levels, J. Atmos. Sci., 36, 2020-2021, 1979.

Winant, C. D., R. C. Beardsley, and R. E. Davis, Moored wind, temperature, and current observations made during Coastal Ocean Dynamics Experiments 1 and 2 over the northern California continental shelf and upper slope, J. Geophys. Res., 92, 1569-1604, 1987.

J. S. Allen, College of Oceanography, Oregon State University, Corvallis, OR 97331.

G. R. Halliwell, Jr., Graduate School of Oceanography, University of Rhode Island, Narragansett, RI 02882.

(Received September 28, 1986; accepted January 5, 1987.) 